\title{
INSTANTONS AND VORTICES ON NONCOMMUTATIVE TORIC VARIETIES
}

\author{
LUCIO S. CIRIO, GIOVANNI LANDI, AND RICHARD J. SZABO
}

\begin{abstract}
We elaborate on the quantization of toric varieties by combining techniques from toric geometry, isospectral deformations and noncommutative geometry in braided monoidal categories, and the construction of instantons thereon by combining methods from noncommutative algebraic geometry and a quantised twistor theory. We classify the real structures on a toric noncommutative deformation of the Klein quadric and use this to derive a new noncommutative four-sphere which is the unique deformation compatible with the noncommutative twistor correspondence. We extend the computation of equivariant instanton partition functions to noncommutative gauge theories with both adjoint and fundamental matter fields, finding agreement with the classical results in all instances. We construct moduli spaces of noncommutative vortices from the moduli of invariant instantons, and derive corresponding equivariant partition functions which also agree with those of the classical limit.
\end{abstract}

\section{Contents}

Introduction $\quad 2$

Acknowledgments $\quad 3$

1. Quantization of toric varieties 3

1.1. Hopf cocycle twist deformations 3

1.2. Cocycle deformations of the complex torus and of $\operatorname{GL}(n) \quad 5$

$\begin{array}{ll}\text { 1.3. Quantum determinants and minors } & 7\end{array}$

1.4. Noncommutative deformations of toric varieties 8

1.5. Noncommutative projective varieties 10

2. Noncommutative instantons 14

2.1. Sheaves on noncommutative toric varieties 14

2.2. Twistor geometry 16

2.3. Real structures on the noncommutative Klein quadric 17

2.4. Noncommutative four-sphere 19

2.5. Twistor bundle on $S_{\theta}^{4} \quad 21$

2.6. Braided ADHM parametrization of instanton moduli 22

2.7. Construction of noncommutative instantons 26

3. Noncommutative gauge theory partition functions 30

3.1. Instanton moduli spaces 30

3.2. Deformations of Hilbert schemes 31

3.3. Equivariant K-theory partition functions 32

3.4. $\mathcal{N}=4$ gauge theory 34

3.5. $\mathcal{N}=2$ gauge theory $\quad 35$

3.6. Adjoint matter fields 35

3.7. Dirac bundles and fundamental matter fields 36

4. Noncommutative vortices 37

Date: December 2012

EMPG-12-25 . 
4.1. Vortices on the projective line 37

4.2. Vortices on the noncommutative plane 39

4.3. Noncommutative vortex counting functions 40

References $\quad 42$

\section{INTRODUCTION}

This paper is a continuation of our ongoing work [5, 6] analysing instanton contributions to gauge theories on noncommutative deformations of algebraic toric varieties. Part of the motivation behind this project was to provide a precise notion of noncommutative toric geometry in which gauge theories could be defined and their corresponding noncommutative instanton solutions constructed. Drawing on the machinery of noncommutative algebraic geometry, the instanton moduli spaces could then be constructed and the corresponding instanton partition functions explicitly evaluated; the goal was to find in this way a rigorous analog of the somewhat heuristic calculations in the physics literature which employ "local" noncommutative deformations to resolve singularities in the instanton moduli spaces and explicitly compute instanton contributions to the partition functions of supersymmetric gauge theories on toric varieties. In [6] we explicitly carried out this construction for framed instantons on the toric noncommutative deformation of the projective plane $\mathbb{C P}^{2}$, and demonstrated that the equivariant instanton partition functions for pure gauge theories coincide with their classical counterparts on $\mathbb{C}^{2}$, thus providing the desired rigorous derivation in the context of noncommutative gauge theory. We stress that although the equivariant instanton counting problems are the same, our instanton moduli spaces are generically (commutative) deformations of those of the classical case; in particular, they potentially provide new examples of quantum integrable systems and could have ramifications in the diverse relationships that exist between fourdimensional supersymmetric gauge theories and field theories in other dimensions, such as two-dimensional conformal field theory. See [18] for further physical motivation and context behind our constructions.

Some of the present article is an overview of the main results and constructions of $[5,6]$ with a somewhat more brief, less technical, and informal style of presentation, which we hope will be more palatable to a wider audience. We also elaborate and extend many of the points from [6]; let us briefly summarise the main new results that the reader will find in this paper.

We shall give a more precise treatment of real structures in our functorial quantization approach, which was glossed over to a certain extent in [6]. In particular, we classify the real structures on a toric noncommutative deformation of the grassmannian $\mathbb{G r}(2 ; 4)$, and hence show that our new deformation of $S^{4}$ found in [6] is the unique noncommutative four-sphere which is compatible with the noncommutative twistor correspondence.

We will also extend the computation of noncommutative instanton partition functions, presented in [6] for the case of pure gauge theories, to gauge theories with matter fields both in the adjoint and fundamental representations of the gauge group. In all cases we confirm precise agreement with the classical results.

Finally, we shall consider the problem of constructing vortices on our noncommutative toric varieties. In the classical case it is well-known that suitable dimensional reductions of 
invariant instantons in four dimensions yield vortices in two dimensions. We will construct noncommutative vortices in our framework via suitable reduction of our instanton moduli constructions and describe their moduli spaces as smooth holomorphic submanifolds of the moduli spaces of noncommutative instantons. We also compute noncommutative vortex partition functions, again including adjoint and fundamental matter fields as well, and once more find agreement in all cases with the classical partition functions. We stress that, as in the case of instanton counting, although the equivariant vortex counting problem is the same as in the classical limit, the vortex moduli spaces are generically (commutative) deformations of the classical ones.

The outline of this paper is as follows. In $\S 1$ we summarise our functorial approach to the quantization of toric varieties, focusing in particular on noncommutative deformations of projective varieties, including projective spaces, grassmannians and flag manifolds as explicit examples. In $\S 2$ we explicitly construct noncommutative instantons in three related incarnations, and give the construction of instanton bundles based on a noncommutative version of the twistor transform. In $\S 3$ we construct smooth moduli spaces for the instanton counting problem on the noncommutative projective plane $\mathbb{C P}_{\theta}^{2}$ and natural bundles over them, and evaluate various equivariant $\mathrm{K}$-theory partition functions for supersymmetric gauge theories on the complex Moyal plane $\mathbb{C}_{\theta}^{2}$. In $\S 4$ we construct moduli spaces for noncommutative vortices, comparing them with those of non-abelian vortices in two-dimensional $\mathcal{N}=(2,2)$ supersymmetric gauge theories, and compute the corresponding noncommutative vortex partition functions via localization in equivariant K-theory.

Acknowledgments. LSC acknowledges support by the National Research Fund, Luxembourg, AFR project 1164566. The work of GL was partially supported by the Italian Project "Cofin08 - Noncommutative Geometry, Quantum Groups and Applications". The work of RJS was supported in part by the Consolidated Grant ST/J000310/1 from the UK Science and Technology Facilities Council, and by Grant RPG-404 from the Leverhulme Trust.

\section{QUANTIZATION OF TORIC VARIETIES}

This section summarizes the algebraic constructions which will be used throughout this paper. We combine constructions from toric geometry and the theory of isospectral deformations, suitably adapted from actions of the real $n$-dimensional torus $\mathbb{T}^{n}$ on riemannian (spin) manifolds to actions of the complex torus $\left(\mathbb{C}^{\times}\right)^{n}$ on algebraic varieties. We use cocycle twist deformations of Hopf algebras and braided monoidal categories of Hopf (co)module algebras as a systematic means of deforming the varieties involved together with geometric objects defined thereon.

1.1. Hopf cocycle twist deformations. Let $\mathcal{H}$ be a Hopf algebra over $\mathbb{C}$ with coproduct $\Delta: \mathcal{H} \rightarrow \mathcal{H} \otimes \mathcal{H}$, counit $\varepsilon: \mathcal{H} \rightarrow \mathbb{C}$, and antipode $S: \mathcal{H} \rightarrow \mathcal{H}$. We use Sweedler notation $\Delta(h)=h_{(1)} \otimes h_{(2)}$, iterated to $(\Delta \otimes \mathrm{id}) \circ \Delta(h)=(\mathrm{id} \otimes \Delta) \circ \Delta(h)=h_{(1)} \otimes h_{(2)} \otimes h_{(3)}$ and so on, with implicit summation. We consider categories of left/right $\mathcal{H}$-modules/comodules, with actions and coactions denoted respectively $\triangleright: \mathcal{H} \otimes V \rightarrow V, \Delta_{V}: V \rightarrow \mathcal{H} \otimes V$, for the left case: for generic $h \in \mathcal{H}$ and $v \in V$ we write $\triangleright(h \otimes v)=h \triangleright v$ and $\Delta_{V}(v)=v^{(-1)} \otimes v^{(0)}$. In a similar way, we use $\triangleleft$ and ${ }_{V} \Delta$ for the right case. When $V$ has some algebraic structure, for example it is an algebra and/or a coalgebra, one can ask for a compatibility condition 
with the $\mathcal{H}$-module/comodule structure by demanding that the maps which define the algebraic structure of $V$ (multiplication, comultiplication, etc.) are morphisms in the category, i.e. they commute with the $\mathcal{H}$-action/coaction. When this is the case we have (left/right) $\mathcal{H}$-module/comodule algebras/coalgebras $V$.

We may deform Hopf algebras and their (co)module categories by a cocycle twist. The construction is due to Drinfel'd, with the dual formulation presented here due to Majid [14]. Given a Hopf algebra $\mathcal{H}$, a linear map $F: \mathcal{H} \otimes \mathcal{H} \rightarrow \mathbb{C}$ is called a Drinfel'd twist element for $\mathcal{H}$ if it is a convolution-invertible unital 2-cocycle on $\mathcal{H}$. These properties ensure that the same coalgebra $\mathcal{H}$, endowed with multiplication and antipode given for $g, h \in \mathcal{H}$ by

$$
\begin{aligned}
g \times_{F} h & =F\left(g_{(1)} \otimes h_{(1)}\right)\left(g_{(2)} \cdot h_{(2)}\right) F^{-1}\left(g_{(3)} \otimes h_{(3)}\right), \\
S^{F}(g) & =U^{F}\left(g_{(1)}\right) S\left(g_{(2)}\right) U^{F-1}\left(g_{(3)}\right) \quad \text { with } \quad U^{F}(g)=F\left(g_{(1)} \otimes S\left(g_{(2)}\right)\right),
\end{aligned}
$$

defines a twisted Hopf algebra structure $\mathcal{H}^{F}$. If $\mathcal{H}$ admits a co(quasi)triangular structure $\mathcal{R}: \mathcal{H} \otimes \mathcal{H} \rightarrow \mathbb{C}$ then $\mathcal{H}^{F}$ is co(quasi)triangular with structure $\mathcal{R}_{F}: \mathcal{H}^{F} \otimes \mathcal{H}^{F} \rightarrow \mathbb{C}$ given by $\mathcal{R}_{F}(g \otimes h)=F\left(h_{(1)} \otimes g_{(1)}\right) \mathcal{R}\left(g_{(2)} \otimes h_{(2)}\right) F^{-1}\left(g_{(3)} \otimes h_{(3)}\right)$.

A Drinfel'd twist for $\mathcal{H}$ naturally induces a deformation of the algebraic structure of $\mathcal{H}$ (co)module (co)algebras. For example if $(A, \mu)$ is a left $\mathcal{H}$-comodule algebra, the deformed product

$$
a \star^{F} b:=F\left(a^{(-1)} \otimes b^{(-1)}\right) \mu\left(a^{(0)} \otimes b^{(0)}\right)
$$

for $a, b \in A$ makes $A^{F}=\left(A, \star^{F}\right)$ into a left $\mathcal{H}^{F}$-comodule algebra.

More generally, Drinfel'd twists naturally affect the whole category of Hopf (co)modules. The induced deformation is encoded in the braiding of the monoidal structure. Let ${ }^{\mathcal{H}} \mathscr{M}$ denote the additive category of left $\mathcal{H}$-comodules. A map $V \stackrel{\sigma}{\longrightarrow} W$ is a morphism of this category if and only if it is $\mathcal{H}$-coequivariant, i.e. $\Delta_{W} \circ \sigma=(\mathrm{id} \otimes \sigma) \circ \Delta_{V}$. The category $\mathcal{H} \mathscr{M}$ has a natural monoidal structure given by the tensor product coaction

$$
\Delta_{V \otimes W}(v \otimes w)=v^{(-1)} w^{(-1)} \otimes\left(v^{(0)} \otimes w^{(0)}\right)
$$

for $v \in V, w \in W$. When $\mathcal{H}$ has the trivial cotriangular structure $\mathcal{R}=\varepsilon \otimes \varepsilon$, the category ${ }^{\mathcal{H}} \mathscr{M}$ of left $\mathcal{H}$-comodules is (trivially) braided by the collection of functorial flip isomorphisms $\Psi_{V, W}(v \otimes w)=w \otimes v$ for each pair of objects $V, W$ of ${ }^{\mathcal{H}} \mathscr{M}$, and for all $v \in V$ and $w \in W$. After deforming $\mathcal{H}$ via a Drinfel'd twist, the twisted cotriangular structure now reads $\mathcal{R}_{F}(g \otimes h)=F\left(h_{(1)} \otimes g_{(1)}\right) F^{-1}\left(g_{(2)} \otimes h_{(2)}\right)$ for every $g, h \in \mathcal{H}$. The monoidal category ${ }^{\mathcal{H}^{F}} \mathscr{M}$ of left $\mathcal{H}^{F}$-comodules is then braided by the collection of functorial isomorphisms

$$
\Psi_{V, W}^{F}(v \otimes w)=\mathcal{R}_{F}\left(w^{(-1)} \otimes v^{(-1)}\right) w^{(0)} \otimes v^{(0)}
$$

for each pair $V, W$ of left $\mathcal{H}^{F}$-comodules with $v \in V$ and $w \in W$. Since $\left(\mathcal{H}^{F}, \mathcal{R}_{F}\right)$ is cotriangular the braiding squares to the identity and makes ${ }^{\mathcal{H}^{F}} \mathscr{M}$ into a braided monoidal category.

If $\left(A, \mu_{A}\right)$ and $\left(B, \mu_{B}\right)$ are left $\mathcal{H}^{F}$-comodule algebras one defines the braided tensor product algebra $A \otimes_{F} B$ to be the vector space $A \otimes B$ endowed with the product map $\mu_{A \otimes_{F} B}:\left(A \otimes_{F} B\right) \otimes\left(A \otimes_{F} B\right) \rightarrow A \otimes_{F} B$ given by

$$
\mu_{A \otimes_{F} B}=\left(\mu_{A} \otimes \mu_{B}\right) \circ\left(\mathrm{id} \otimes \Psi_{B, A}^{F} \otimes \mathrm{id}\right) .
$$

The resulting algebra is an object of the category ${ }^{\mathcal{H}^{F}} \mathscr{M}$ by the tensor product coaction. 
The deformation in passing from $\mathcal{H}$ to $\mathcal{H}^{F}$ takes the form of a functorial isomorphism $\mathscr{F}_{F}:{ }^{\mathcal{H}} \mathscr{M} \rightarrow{ }^{\mathcal{H}^{F}} \mathscr{M}$ of braided monoidal categories. The functor $\mathscr{F}_{F}$ acts as the identity on objects and morphisms of ${ }^{\mathcal{H}} \mathscr{M}$, but defines a new monoidal structure,

$$
\lambda_{F}: \mathscr{F}_{F}(V) \otimes \mathscr{F}_{F}(W) \longrightarrow \mathscr{F}_{F}(V \otimes W), \quad \lambda_{F}(v \otimes w)=F\left(v^{(-1)} \otimes w^{(-1)}\right) v^{(0)} \otimes w^{(0)},
$$

on the category ${ }^{\mathcal{H}_{F}} \mathscr{M}$. This makes $\mathscr{F}_{F}$ into a monoidal functor intertwining the braidings in ${ }^{\mathcal{H}} \mathscr{M}$ and ${ }^{\mathcal{H}_{F}} \mathscr{M}$, given respectively by the flip functor $\Psi$ and the functor $\Psi^{F}$ in (1.3).

Since later on we shall also touch upon $*$-structures, we recall that a $*$-algebra is an algebra $A$ endowed with an involutive anti-linear anti-homomorphism $*: A \rightarrow A$. A Hopf *-algebra is a Hopf algebra $\mathcal{H}$ with a $*$-structure such that for every $h \in \mathcal{H}$ one has

$$
\Delta\left(h^{*}\right)=\left(h_{(1)}\right)^{*} \otimes\left(h_{(2)}\right)^{*}, \quad \varepsilon\left(h^{*}\right)=\overline{\varepsilon(h)} .
$$

In general one also requires $(S \circ *)^{2}=\mathrm{id}$; this is however redundant with an invertible antipode since then $* \circ S^{-1} \circ *$ satisfies all the conditions for the antipode and the latter is unique.

For a left action of a Hopf *-algebra $\mathcal{H}$ on a $*$-algebra $A$, with involution denoted $\dagger: A \rightarrow A$, one requires compatibility, i.e. that the action be a $*$-action, so that for $h \in \mathcal{H}, a \in A$, one has

$$
h \triangleright a^{\dagger}=\left(S(h)^{*} \triangleright a\right)^{\dagger}
$$

Similarly, for a left coaction one requires it to be a $*$-coaction, i.e. for $a \in A$ one has

$$
\Delta_{A}\left(a^{\dagger}\right)=\left(a^{(-1)}\right)^{*} \otimes\left(a^{(0)}\right)^{\dagger} .
$$

When deforming via a Drinfel'd twist $F$ as before, if $\mathcal{H}$ is a Hopf $*$-algebra with invertible antipode (as will always be the case in the present paper) the only condition for the deformation $\mathcal{H}^{F}$ to be a Hopf $*$-algebra with the same $*$-structure is $\left(g \times_{F} h\right)^{*}=h^{*} \times_{F} g^{*}$, which forces the Drinfel'd twist $F$ to be real, i.e. to satisfy

$$
\overline{F(g \otimes h)}=F\left(h^{*} \otimes g^{*}\right)
$$

for all $g, h \in \mathcal{H}$. If $(A, \mu)$ is a left $\mathcal{H}$-comodule $*$-algebra, the $*$-structure of $A$ makes $A^{F}$ into a *-algebra if and only if the Drinfel'd twist is real; in addition, $A^{F}$ becomes an $\mathcal{H}^{F}$-comodule $*$-algebra.

Right analogs of all the structures above are straightforwardly constructed.

1.2. Cocycle deformations of the complex torus and of $\mathrm{GL}(n)$. We deform the coordinate algebra of the complex torus $T=\left(\mathbb{C}^{\times}\right)^{n}$ via the cocycle twist procedure. The Pontrjagin dual group $\widehat{T}=\operatorname{Hom}_{\mathbb{C}}\left(T, \mathbb{C}^{\times}\right) \cong \mathbb{Z}^{n}$ is the group of characters of the group $T$. For $p=\left(p_{1}, \ldots, p_{n}\right) \in \mathbb{Z}^{n}$ and $t=\left(t_{1}, \ldots, t_{n}\right) \in T$, the characters are given by

$$
t^{p}:=t_{1}^{p_{1}} \cdots t_{n}^{p_{n}}
$$

The unital algebra $\mathcal{H}=\mathcal{A}(T)$ of coordinate functions on the torus $T$ is the Laurent polynomial algebra $\mathcal{H}:=\mathbb{C}\left(t_{1}, \ldots, t_{n}\right)$ of characters. It has Hopf algebra structure

$$
\Delta\left(t^{p}\right)=t^{p} \otimes t^{p}, \quad \varepsilon\left(t^{p}\right)=1, \quad S\left(t^{p}\right)=t^{-p}
$$

for $p \in \mathbb{Z}^{n}$, with the coproduct and counit extended as algebra morphisms and the antipode as an anti-algebra morphism. The canonical right action of $T$ on itself by group multiplication dualizes to a left $\mathcal{H}$-coaction

$$
\Delta_{\mathcal{A}(T)}: \mathcal{A}(T) \longrightarrow \mathcal{H} \otimes \mathcal{A}(T), \quad \Delta_{\mathcal{A}(T)}\left(u_{i}^{ \pm 1}\right)=t_{i}^{ \pm 1} \otimes u_{i}^{ \pm 1}
$$


where we write $u_{i}^{ \pm 1}, i=1, \ldots, n$, for the generators of $\mathcal{A}(T)$ viewed as a left comodule algebra over itself, to distinguish the coordinate algebra $\mathcal{A}(T)$ from the Hopf algebra $\mathcal{H}$.

To deform the Hopf algebra $\mathcal{H}$ by a Drinfel'd twist we choose a complex skew-symmetric $n \times n$ matrix $\theta=\left(\theta^{i j}\right)$ and on characters $t^{p}, t^{q} \in \mathcal{H}$ we set

$$
F_{\theta}\left(t^{p} \otimes t^{q}\right)=\exp \left(\frac{\mathrm{i}}{2} \sum_{i, j=1}^{n} p_{i} \theta^{i j} q_{j}\right),
$$

and extend it by linearity. Then $F_{\theta}$ is completely determined by its values on generators

$$
F_{\theta}\left(t_{i} \otimes t_{j}\right)=\exp \left(\frac{\mathrm{i}}{2} \theta^{i j}\right)=: q_{i j}, \quad i, j=1, \ldots, n .
$$

One easily checks that $F_{\theta}$ is a Drinfel'd twist element for $\mathcal{H}$ and that $\mathcal{H}_{\theta}:=\mathcal{H}^{F_{\theta}}=\mathcal{H}$ as a Hopf algebra. However, $\mathcal{H}$ and $\mathcal{H}_{\theta}$ differ as cotriangular Hopf algebras: while $\mathcal{H}$ has trivial cotriangular structure $\mathcal{R}=\varepsilon \otimes \varepsilon$, the twisted cotriangular structure on $\mathcal{H}_{\theta}$ is given by

$$
\mathcal{R}_{\theta}\left(t_{i} \otimes t_{j}\right)=F_{\theta}\left(t_{j} \otimes t_{i}\right) F_{\theta}^{-1}\left(t_{i} \otimes t_{j}\right)=F_{\theta}^{-2}\left(t_{i} \otimes t_{j}\right)=q_{i j}^{-2} .
$$

Then $\mathcal{A}(T)$, regarded as an $\mathcal{H}$-comodule algebra, acquires by (1.2) a deformed noncommutative product $u_{i} \star_{\theta} u_{j}=F_{\theta}\left(t_{i} \otimes t_{j}\right) u_{i} u_{j}=q_{i j} u_{i} u_{j}$ which corresponds to relations

$$
u_{i} \star_{\theta} u_{j}=q_{i j}^{2} u_{j} \star_{\theta} u_{i}, \quad u_{i}^{-1} \star_{\theta} u_{j}=q_{i j}^{-2} u_{j} \star_{\theta} u_{i}^{-1} \quad \text { for } \quad i, j=1, \ldots, n \text {. }
$$

The algebra $\mathcal{A}\left(T_{\theta}\right):=\left(\mathcal{A}(T), \star_{\theta}\right)=\mathbb{C}_{\theta}\left(t_{1}, \ldots, t_{n}\right)$ is dual to a noncommutative variety denoted $T_{\theta}=\left(\mathbb{C}_{\theta}^{\times}\right)^{n}$ and called the noncommutative complex torus. We stress that the deformation affects only the algebra structure of $\mathcal{A}(T)$, not the Hopf algebra structure of $\mathcal{H}$. This means we deform the complex torus only as a space, not as a group, as in the case of the noncommutative real torus.

We can also define a noncommutative toric deformation of the complex general linear group GL $(n)$ realized again by a Drinfel'd twist. Consider the Hopf algebra of coordinate functions $\mathcal{F}_{n}:=\operatorname{Fun}(\mathrm{GL}(n))$, with generators $g_{i j}, i, j=1, \ldots, n$. We identify the generators $t_{i} \in \mathcal{H}$ with $g_{i i} \in \mathcal{F}_{n}$ (dual to the embedding $\left(\mathbb{C}^{\times}\right)^{n} \hookrightarrow \operatorname{GL}(n)$ ), so that the twist element (1.6) which deforms the complex torus is a Drinfel'd twist for $\mathcal{F}_{n}$ too. We have

$$
F_{\theta}\left(g_{i j} \otimes g_{k l}\right)=\exp \left(\frac{\mathrm{i}}{2} \theta^{i k}\right) \delta_{i j} \delta_{k l}=q_{i k} \delta_{i j} \delta_{k l},
$$

yielding a new Hopf algebra $\mathcal{F}_{n}^{\theta}$ with twisted multiplication as in (1.1). Introducing coefficients

$$
Q_{i j ; k l}=q_{k i} q_{j l}=q_{i k}^{-1} q_{j l}, \quad Q_{i j ; k l}^{2}=q_{k i}^{2} q_{j l}^{2}
$$

the commutation rules for the generators of $\mathcal{F}_{n}^{\theta}$ are computed to be

$$
g_{i j} \times_{\theta} g_{k l}=Q_{i j ; k l}^{2} g_{k l} \times_{\theta} g_{i j} .
$$

While the coproduct $\Delta\left(g_{i j}\right)=\sum_{k} g_{i k} \otimes g_{k j}$ and the counit $\varepsilon\left(g_{i j}\right)=\delta_{i j}$ are left unchanged by the general procedure, direct computations show that the antipode $S\left(g_{i j}\right)=\left(g^{-1}\right)_{i j}$ is unaltered as well.

The noncommutative Hopf algebra $\mathcal{F}_{n}^{\theta}=\left(\mathcal{F}_{n}, \times_{\theta}, \Delta, \varepsilon, S\right)$ is called the complex torus deformation quantum group of $\mathrm{GL}(n)$. It is dual to a noncommutative variety which we denote by $\mathrm{GL}_{\theta}(n)$. 
1.3. Quantum determinants and minors. The coordinate algebra of $\mathrm{GL}_{\theta}(n)$ should be properly defined as the Ore localization of the noncommutative algebra generated by arbitrary matrix units with respect to an invertible and permutable element $\operatorname{det}_{\theta}$, the determinant element. For a generic $n \times n$ matrix whose entries $g_{i j}$ satisfy commutation relations (1.9) we adapt the Leibniz formula which expresses the determinant as a linear combination of products $\prod_{i} g_{i \sigma(i)}$ or $\prod_{i} g_{\sigma(i) i}$ as the permutation $\sigma$, weighted by its sign, runs through the symmetric group $S_{n}$. In order to get a well defined element of $\mathcal{F}_{n}^{\theta}$ we put a factor $Q_{l j ; k i}$ for every pair $g_{k i} g_{l j}$ appearing in $\prod_{i} g_{i \sigma(i)}$. However, a more succinct expression is achieved via a $\theta$-deformed Levi-Civita symbol $\epsilon_{\theta}$. Since the row and column indices in (1.8) and (1.9) behave differently, we actually need to introduce two different symbols $\epsilon_{\theta}^{(r)}$, which refers to row indices, and $\epsilon_{\theta}^{(c)}$, which refers to column indices; they are given by

$$
\begin{aligned}
& \epsilon_{\theta}^{i_{1} \cdots i_{n}(c)}=\operatorname{sgn}\left(i_{1} \cdots i_{n}\right) \prod_{k=1}^{n-1} \prod_{s=1}^{n-k} Q_{s+k i_{s+k} ; k i_{k}}, \\
& \epsilon_{\theta}^{j_{1} \cdots j_{n}(r)}=\operatorname{sgn}\left(j_{1} \cdots j_{n}\right) \prod_{k=1}^{n-1} \prod_{s=1}^{n-k} Q_{j_{s+k} s+k ; j_{k} k} .
\end{aligned}
$$

They obey the alternating rules

$$
\begin{aligned}
& \epsilon_{\theta}^{j_{1} \cdots j_{\alpha} \cdots j_{\beta} \cdots j_{n}(c)}=-q_{j_{\alpha} j_{\beta}}^{-2} \epsilon_{\theta}^{j_{1} \cdots j_{\beta} \cdots j_{\alpha} \cdots j_{n}(c)}, \\
& \epsilon_{\theta}^{i_{1} \cdots i_{\alpha} \cdots i_{\beta} \cdots i_{n}(r)}=-q_{i_{\alpha} i_{\beta}}^{2} \epsilon_{\theta}^{i_{1} \cdots i_{\beta} \cdots i_{\alpha} \cdots i_{n}(r)},
\end{aligned}
$$

and the symbol $\epsilon_{\theta}^{(r)}$ may be thought of as the inverse of the symbol $\epsilon_{\theta}^{(c)}$. Using them we may absorb the $Q$-dependent factors.

The quantum determinant is then the element of $\mathcal{F}_{n}^{\theta}$ given by

$$
\operatorname{det}_{\theta}=\frac{1}{n !} \sum_{i_{k}, j_{k}=1}^{n} \epsilon_{\theta}^{i_{1} \cdots i_{n}(r)} \epsilon_{\theta}^{j_{1} \cdots j_{n}(c)} g_{i_{1} j_{1}} \cdots g_{i_{n} j_{n}} .
$$

Note that $\operatorname{det}_{\theta}$ is central in $\mathcal{F}_{n}^{\theta}$ if and only if $\sum_{k=1}^{n} \theta^{k i}=\sum_{k=1}^{n} \theta^{k j}$, modulo $2 \pi$, for all $i, j=1, \ldots, n$. On the other hand, the element $\operatorname{det}_{\theta}$ is an $\mathcal{H}_{\theta}$-coeigenvector which is left and right permutable in $\mathcal{F}_{n}^{\theta}$. This means that its non-negative integer powers form a left and right denominator set in $\mathcal{F}_{n}^{\theta}$, and hence we can perform noncommutative Ore localization with respect to $\operatorname{det}_{\theta}$.

The determinant element (1.11) originates from the braiding $\Psi^{\theta}$ of the category of left $\mathcal{F}_{n}^{\theta}$-comodules ${ }^{\mathcal{F}_{n}^{\theta}} \mathscr{M}$ given, in the present case, by (1.3) and (1.7). The $\theta$-deformed exterior algebra of degree $d$ for an object $V$ in ${ }^{\mathcal{F}_{n}^{\theta}} \mathscr{M}$ is defined as

$$
\bigwedge_{\theta}^{d} V:=V^{\otimes d} /\left\langle v_{1} \otimes v_{2}+\Psi_{V, V}^{\theta}\left(v_{1} \otimes v_{2}\right)\right\rangle_{v_{1}, v_{2} \in V} .
$$

The braided skew-symmetric algebra $\bigwedge_{\theta}^{d} V$ is spanned by the collection of minors of order $d \leq n$ in elements of $V$ when $n$ is the number of generators of $V$. Indeed consider two multi-indices $I=\left(i_{1} \cdots i_{d}\right)$ and $J=\left(j_{1} \cdots j_{d}\right)$ labelling the rows and columns of a given minor, and define the determinant $\Lambda^{I J}$ of this sub-matrix as

$$
\Lambda^{I J}=\frac{1}{d !} \sum_{i_{k} \in I, j_{k} \in J} \epsilon_{\theta}^{i_{1} \cdots i_{d}(r)} \epsilon_{\theta}^{j_{1} \cdots j_{d}(c)} g_{i_{1} j_{1}} \cdots g_{i_{d} j_{d}}
$$


where the symbols $\epsilon_{\theta}$ satisfy alternating rules derived from (1.10). Here the $\mathcal{F}_{n}^{\theta}$-comodule structure of $\mathrm{GL}(n)$ is induced from the $\mathcal{F}_{n}^{\theta}$-comodule structure of $V$ and of its dual $V^{*}$. When this $\mathcal{F}_{n}^{\theta}$-comodule structure induces the noncommutative product (1.9) among the entries of elements of GL $(n)$, the alternating properties of the deformed Levi-Civita symbols coincide with those of (1.10).

For later use we introduce a deformation of the Laplace expansion of the determinant in terms of lower order minors. If $I$ is a row multi-index, $J$ a column multi-index with $|I|=|J|=d$ we write $I^{\alpha}=I \backslash\left\{i_{\alpha}\right\}=\left(i_{1}^{\alpha}, \ldots, i_{d-1}^{\alpha}\right)$ and $J^{\alpha}=J \backslash\left\{j_{\alpha}\right\}=\left(j_{1}^{\alpha}, \ldots, j_{d-1}^{\alpha}\right)$ for $\alpha \in(1, \ldots, d)$. By taking into account the appropriate $Q$-factors, the noncommutative version of the classical expansion of the determinant $\Lambda^{I J}$ with respect to the $k$-th row is

$$
\Lambda^{I J}=\sum_{\alpha=1}^{d} \prod_{\beta=1}^{d-1}(-1)^{k+\alpha} Q_{i_{\beta}^{k} j_{\beta}^{\alpha} ; k \alpha} g_{k \alpha} \Lambda^{I^{k} J^{\alpha}}=\sum_{\alpha=1}^{d} \prod_{\beta=1}^{d-1}(-1)^{k+\alpha} Q_{k \alpha ; i_{\beta}^{k} j_{\beta}^{\alpha}} \Lambda^{I^{k} J^{\alpha}} g_{k \alpha}
$$

Similarly, if we expand with respect to the $k$-th column we get

$$
\Lambda^{I J}=\sum_{\alpha=1}^{d} \prod_{\beta=1}^{d-1}(-1)^{k+\alpha} Q_{i_{\beta}^{k} j_{\beta}^{\alpha} ; \alpha k} g_{\alpha k} \Lambda^{I^{\alpha} J^{k}}=\sum_{\alpha=1}^{d} \prod_{\beta=1}^{d-1}(-1)^{k+\alpha} Q_{\alpha k ; i_{\beta}^{k} j_{\beta}^{\alpha}} \Lambda^{I^{\alpha} J^{k}} g_{\alpha k}
$$

We will also need the commutation rules between any two $d \times d$ and $d^{\prime} \times d^{\prime}$ minors $\Lambda^{I J}$ and $\Lambda^{I^{\prime} J^{\prime}}$, when $\mathcal{F}_{n}^{\theta}$ is regarded as the coordinate algebra $\mathcal{A}\left(\mathrm{GL}_{\theta}(n)\right)$ of the noncommutative variety $\mathrm{GL}_{\theta}(n)$. A direct computation gives

$$
\Lambda^{I J} \Lambda^{I^{\prime} J^{\prime}}=R_{I J ; I^{\prime} J^{\prime}}^{2} \Lambda^{I^{\prime} J^{\prime}} \Lambda^{I J}
$$

where we introduced the commutation coefficient

$$
R_{I J ; I^{\prime} J^{\prime}}=\prod_{\alpha=1}^{d} \prod_{\alpha^{\prime}=1}^{d^{\prime}} Q_{i_{\alpha} j_{\alpha} ; i_{\alpha^{\prime}}^{\prime} j_{\alpha^{\prime}}^{\prime}} .
$$

In particular, this shows that the minors of order $d$ generate a subalgebra.

Finally, consider a pair of multi-indices differing only by transposition of two columns, $J=\left(j_{1} \cdots j_{\alpha} \cdots j_{\beta} \cdots j_{d}\right)$ and $J^{t_{\alpha \beta}}=\left(j_{1} \cdots j_{\beta} \cdots j_{\alpha} \cdots j_{d}\right)$. From (1.13) one obtains

$$
\Lambda^{I J}=(-1)^{\beta-\alpha} \Lambda^{I J^{t} \alpha \beta}
$$

which are alternating relations that can be further generalized to arbitrary permutations.

1.4. Noncommutative deformations of toric varieties. A toric variety $X$ of dimension $n$ is an irreducible algebraic variety over $\mathbb{C}$ which contains the complex torus $T=\left(\mathbb{C}^{\times}\right)^{n}$ as a Zariski open subset and the regular action of $T$ on itself extends to an action on the whole of $X$. Basic examples are the complex torus $T$ itself, the affine planes $\mathbb{C}^{n}$, the projective spaces $\mathbb{C P}^{n}$, and the weighted projective spaces $\mathbb{C P}^{n}\left[a_{0}, a_{1}, \ldots, a_{n}\right]$.

One of the main interests in studying toric varieties is that their geometry can be encoded by combinatorial data, a fan, that describes the way in which $T$ acts on $X$. Various properties of a toric variety, such as smoothness and compactness, may be formulated entirely in terms of the fan structure. We will quantize toric varieties by using the same fan description and deforming the coordinate algebra of every cone of the fan.

Recall that a rational polyhedral cone $\sigma \subset \mathbb{R}^{n}$ is a cone $\sigma=\mathbb{R}_{\geq 0} v_{1}+\cdots+\mathbb{R}_{\geq 0} v_{s}$ generated by finitely many elements $v_{1}, \ldots, v_{s} \in \mathbb{Z}^{n}$. It is strongly convex if it does not contain any real line, $\sigma \cap(-\sigma)=0$. For every strongly convex rational polyhedral cone 
$\sigma \subset \mathbb{R}^{n}$ we define the dual cone $\sigma^{\vee}:=\left\{m \in \mathbb{R}^{n} \mid m \cdot u \geq 0 \quad \forall u \in \sigma\right\}$. The set $\sigma^{\vee} \cap \mathbb{Z}^{n}$ is a finitely generated semigroup under addition, with generators $\left(m_{1}, \ldots, m_{l}\right)$ where $l \geq n$. To each $m_{a}=\left(\left(m_{a}\right)_{1}, \ldots,\left(m_{a}\right)_{n}\right)$ one associates a Laurent monomial in $\mathbb{C}\left(t_{1}, \ldots, t_{n}\right)$ by the assignment $m_{a} \mapsto t^{m_{a}}=t_{1}^{\left(m_{a}\right)_{1}} \cdots t_{n}^{\left(m_{a}\right)_{n}}$. The product between any two such elements is via the corresponding sum of characters, $t^{m_{a}} \cdot t^{m_{b}}:=t^{m_{a}+m_{b}}$. Thus the generators of $\sigma^{\vee} \cap \mathbb{Z}^{n}$ span a subalgebra of $\mathbb{C}\left(t_{1}, \ldots, t_{n}\right)$ denoted $\mathbb{C}[\sigma]$, the coordinate algebra of a normal affine toric variety $U[\sigma]=\operatorname{Spec}(\mathbb{C}[\sigma])$. Note that the inclusion $0 \hookrightarrow \sigma$ induces an embedding of the torus $T=U[0]$ as a dense open subset of $U[\sigma]$.

Our noncommutative deformation is induced by the cocycle deformation of this complex torus. It starts with the same complex skew-symmetric matrix $\theta$ as in (1.6). The algebra $\mathbb{C}_{\theta}[\sigma]$ is defined to be the subalgebra of $\mathbb{C}_{\theta}\left(t_{1}, \ldots, t_{n}\right)$ generated by $\left\{t^{m_{a}}\right\}$ with product

$$
t^{m_{a}} \star_{\theta} t^{m_{b}}:=\exp \left(\frac{\mathrm{i}}{2} \sum_{i, j=1}^{n}\left(m_{a}\right)_{i} \theta^{i j}\left(m_{b}\right)_{j}\right) t^{m_{a}+m_{b}} .
$$

This corresponds to a deformation of the algebra generated by the characters, but not of their group structure. It is a finitely-generated $\mathcal{H}_{\theta}$-comodule subalgebra of the algebra $\mathcal{A}\left(T_{\theta}\right)$ of the noncommutative torus. The noncommutative affine variety corresponding to the algebra $\mathbb{C}_{\theta}[\sigma]$ is denoted $U_{\theta}[\sigma]$. It is a multi-parameter deformation of $U[\sigma]$.

The variety $U[\sigma]$ may also be described as an embedding in the complex plane $\mathbb{C}^{l}$. If $\sigma^{\vee} \cap \mathbb{Z}^{n}$ has $l$ generators, consider the polynomial algebra $\mathbb{C}\left[x_{1}, \ldots, x_{l}\right]$ (one variable $x_{a}$ for each $m_{a}$ ). Since the generators $m_{a}$ are $l$ rational vectors in $\mathbb{R}^{n}$, there are $l-n$ linear relations among them. Then we may quotient the algebra $\mathbb{C}\left[x_{1}, \ldots, x_{l}\right]$ by the ideal generated by the $l-n$ relations for the vectors $m_{a}$, realized as multiplicative relations for the variables $x_{a}$. If $R\left[m_{a}\right] \subset \mathbb{C}\left[x_{1}, \ldots, x_{l}\right]$ denotes the subspace generated by these relations, then $U[\sigma]$ is realized as the spectrum of the quotient algebra $\mathbb{C}[\sigma]=\mathbb{C}\left[x_{1}, \ldots, x_{l}\right] /\left\langle R\left[m_{a}\right]\right\rangle$. An analogous realization is possible for noncommutative affine toric varieties. The noncommutative deformation of the polynomial algebra $\mathbb{C}\left[x_{1}, \ldots, x_{l}\right]$ is obtained from the multiplicative relations between the Laurent monomials $t^{m_{a}}$. These relations become

$$
t^{m_{a}} \star_{\check{\theta}} t^{m_{b}}:=\check{q}_{a b} t^{m_{a}+m_{b}},
$$

where we denote $\check{\theta}_{a b}:=\sum_{i, j}\left(m_{a}\right)_{i} \theta^{i j}\left(m_{b}\right)_{j}$ and $\check{q}_{a b}=\exp \left(\frac{\mathrm{i}}{2} \check{\theta}_{a b}\right)$ with $a, b=1, \ldots, l$, and $i, j=1, \ldots, n$. Then the generators of the algebra of the affine variety obey

$$
x_{a} \star_{\check{\theta}} x_{b}=\left(\check{q}_{a b}\right)^{2} x_{b} \star_{\check{\theta}} x_{a} .
$$

These relations define the $l$-dimensional complex Moyal plane $\mathbb{C}_{\check{\theta}}^{l}$ with coordinate algebra $\mathbb{C}_{\check{\theta}}\left[x_{1}, \ldots, x_{l}\right]$. The $l-n$ linear relations among the generators of the dual cone $\left\{m_{a}\right\}$ are now expressed in the character algebra. They can always be brought to the form

$$
\sum_{a=1}^{l}\left(p_{s, a}-r_{s, a}\right) m_{a}=0 \quad \text { for } \quad s=1, \ldots, l-n,
$$

with non-negative integer coefficients $p_{s, a}$ and $r_{s, a}$. Then for each $s$, one obtains from (1.19) the additional relation

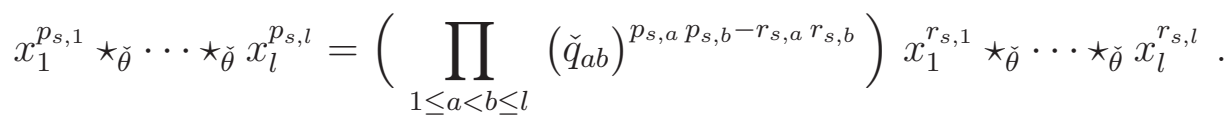

The subspace of relations (1.21) is denoted $R_{\breve{\theta}}\left[m_{a}\right]$. It is a multi-parameter deformation of the subspace $R\left[m_{a}\right]$, which generates a two-sided ideal in $\mathbb{C}_{\breve{\theta}}\left[x_{1}, \ldots, x_{l}\right]$. The quotient 
algebra $\mathbb{C}_{\check{\theta}}\left[x_{1}, \ldots, x_{l}\right] /\left\langle R_{\check{\theta}}\left[m_{a}\right]\right\rangle$ is an alternative realization of the coordinate algebra $\mathbb{C}_{\theta}[\sigma]$ of the noncommutative affine variety $U_{\theta}[\sigma]$.

We obtain generic toric varieties by gluing together affine toric varieties. For this, we recall the notion of a fan. Given a cone $\sigma \subset \mathbb{R}^{n}$, a face $\tau \subset \sigma$ is a subset of the form $\tau=\sigma \cap m^{\perp}$ for some $m \in \sigma^{\vee}$, where $m^{\perp}:=\left\{u \in \mathbb{R}^{n} \mid m \cdot u=0\right\}$. A fan $\Sigma \subset \mathbb{R}^{n}$ is a non-empty finite collection of strongly convex rational polyhedral cones in $\mathbb{R}^{n}$ satisfying two conditions: firstly, if $\sigma \in \Sigma$ and $\tau$ is a face of $\sigma$, then $\tau \in \Sigma$; secondly, if $\sigma, \sigma^{\prime} \in \Sigma$, then the intersection $\sigma \cap \sigma^{\prime}$ is a face of both $\sigma$ and $\sigma^{\prime}$. To a fan $\Sigma$ in $\mathbb{R}^{n}$ we associate a toric variety $X=X[\Sigma]$. The cones $\sigma \in \Sigma$ correspond to the open affine subvarieties $U[\sigma] \subset X[\Sigma]$, and $U[\sigma]$ and $U\left[\sigma^{\prime}\right]$ are glued together along their common open subset $U\left[\sigma \cap \sigma^{\prime}\right]=U[\sigma] \cap U\left[\sigma^{\prime}\right]$.

In a similar way, we obtain generic noncommutative toric varieties $X_{\theta}[\Sigma]$ by gluing together noncommutative affine toric varieties. If $\sigma$ and $\sigma^{\prime}$ are two cones in the fan $\Sigma$ which intersect along the face $\tau=\sigma \cap \sigma^{\prime}$, then there are canonical morphisms between the associated noncommutative algebras $\mathbb{C}_{\theta}[\sigma] \rightarrow \mathbb{C}_{\theta}[\tau]$ and $\mathbb{C}_{\theta}\left[\sigma^{\prime}\right] \rightarrow \mathbb{C}_{\theta}[\tau]$ induced by the inclusions $\tau \hookrightarrow \sigma$ and $\tau \hookrightarrow \sigma^{\prime}$. The images of these morphisms in $\mathbb{C}_{\theta}[\tau]$ are related by an algebra automorphism in the category ${ }^{\mathcal{H}_{\theta}} \mathscr{M}$ playing the role of a "coordinate transition function" between $U_{\theta}[\sigma]$ and $U_{\theta}\left[\sigma^{\prime}\right]$. See $[5, \S 3.1]$ for the explicit description in the general case, and $\S 1.5$ below for the example of the noncommutative projective plane.

Since each algebra $\mathbb{C}_{\theta}[\sigma]$ for $\sigma \in \Sigma$ is a subalgebra of $\mathbb{C}_{\theta}\left(t_{1}, \ldots, t_{n}\right)$, the intersection of the algebras $\mathbb{C}_{\theta}[\sigma]$ is well-defined, and the "algebra of coordinate functions" $\mathcal{A}\left(X_{\theta}[\Sigma]\right)$ on $X_{\theta}[\Sigma]$ can be represented via the exact sequence

$$
0 \longrightarrow \mathcal{A}\left(X_{\theta}[\Sigma]\right) \longrightarrow \prod_{\sigma \in \Sigma} \mathbb{C}_{\theta}[\sigma] \longrightarrow \prod_{\sigma, \sigma^{\prime} \in \Sigma} \mathbb{C}_{\theta}\left[\sigma \cap \sigma^{\prime}\right]
$$

with the gluing automorphisms discussed above. The sequence (1.22) is an exact sequence in the category ${ }^{\mathcal{H}_{\theta}} \mathscr{M}$, and hence $\mathcal{A}\left(X_{\theta}[\Sigma]\right)$ is a left $\mathcal{H}_{\theta}$-comodule algebra.

1.5. Noncommutative projective varieties. We specialize to noncommutative projective spaces $\mathbb{C P}_{\theta}^{n}$. These varieties admit also a "global" description via noncommutative homogeneous coordinate algebras which, after Ore localization, reduces to the local description provided by the noncommutative affine varieties $U_{\theta}[\sigma]$. Moreover, they are used to define noncommutative deformations of projective varieties, such as grassmannians, via restriction.

Noncommutative projective plane. Consider lattice vectors $v_{1}=(1,0), v_{2}=(0,1)$ and $v_{3}=(-1,-1)$ in $\mathbb{Z}^{2}$. The fan $\Sigma_{\mathbb{C P}^{2}}$ of $\mathbb{C P}^{2}$ has one-dimensional cones $\tau_{i}=\mathbb{R}_{\geq 0} v_{i}$, while the three maximal cones are generated by pairs of these vectors as $\sigma_{i}=\mathbb{R}_{\geq 0} v_{i+1}+\mathbb{R}_{\geq 0} v_{i+2}$ (with indices $i=1,2,3$ read $\bmod 3$ ) with $\sigma_{i} \cap \sigma_{i+1}=\tau_{i+2}$ and $\sigma_{i} \cap \sigma_{j}=0$ otherwise. The zero cone is the triple overlap $\sigma_{1} \cap \sigma_{2} \cap \sigma_{3}=0$. Pictorially

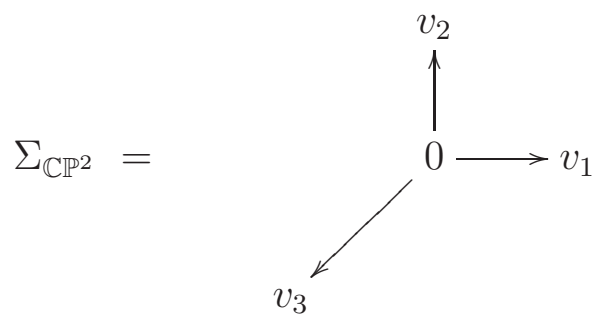


The corresponding open affine subvarieties $U\left[\sigma_{i}\right]$ generate an open cover of $X\left[\Sigma_{\mathbb{C P}^{2}}\right]=\mathbb{C P}^{2}$.

For the noncommutative deformation we write out the relations among the generators of the subalgebras $\mathbb{C}_{\theta}\left[\sigma_{i}\right] \subset \mathbb{C}_{\theta}\left(t_{1}, t_{2}\right)$. Each dual cone $\sigma_{i}^{\vee}$ is strongly convex and hence the generators of $\sigma_{i}^{\vee} \cap \mathbb{Z}^{2}$ are independent. The semigroup $\sigma_{3}^{\vee} \cap \mathbb{Z}^{2}$ is generated by $m_{1}=(1,0)$ and $m_{2}=(0,1)$, so that $\check{\theta}=\theta:=\theta^{12}$ for the deformation matrix, and the algebra $\mathbb{C}_{\theta}\left[\sigma_{3}\right]=\mathbb{C}_{\theta}\left[x_{1}, x_{2}\right]$ is generated by $x_{a}=t^{m_{a}}=t_{a}, a=1,2$, with the relation

$$
x_{1} x_{2}=q^{2} x_{2} x_{1}
$$

where $q:=q_{12}$. The other two cones $\sigma_{i}$ for $i=1,2$ are similarly treated and, after suitable redefinitions of the generators, in each case one finds $\check{\theta}=\theta$ and that $\mathbb{C}_{\theta}\left[\sigma_{i}\right]$ is generated by elements $x_{1}, x_{2}$ satisfying the relations (1.23). All three varieties $U_{\theta}\left[\sigma_{i}\right] \cong \mathbb{C}_{\theta}^{2}$ are thus copies of the two-dimensional complex Moyal plane.

To glue the noncommutative affine toric varieties together, consider for example the face $\tau_{1}=\sigma_{3} \cap \sigma_{1}$. The semigroup $\tau_{1}^{\vee} \cap \mathbb{Z}^{2}$ is generated by $m_{1}=(1,0), m_{2}=(0,1)$ and $m_{3}=-m_{2}$. The generators of the subalgebra $\mathbb{C}_{\theta}\left[\tau_{1}\right]=\mathbb{C}_{\theta}\left[t_{1}, t_{2}, t_{2}^{-1}\right]$ are the elements $y_{1}=t_{1}, y_{2}=t_{2}$ and $y_{3}=t_{2}^{-1}$ with the relations

$$
y_{1} y_{2}=q^{2} y_{2} y_{1}, \quad y_{1} y_{3}=q^{-2} y_{3} y_{1}, \quad y_{2} y_{3}=1=y_{3} y_{2},
$$

which we may identify as the algebra dual to a noncommutative projective line $\mathbb{C P}_{\theta}^{1}$. The algebra morphisms $\mathbb{C}_{\theta}\left[\sigma_{1}\right] \rightarrow \mathbb{C}_{\theta}\left[\tau_{1}\right]$ and $\mathbb{C}_{\theta}\left[\sigma_{3}\right] \rightarrow \mathbb{C}_{\theta}\left[\tau_{1}\right]$ are both natural inclusions of subalgebras, and in this manner there is a natural algebra automorphism $\mathbb{C}_{\theta}\left[\sigma_{1}\right] \rightarrow \mathbb{C}_{\theta}\left[\sigma_{3}\right]$. The other faces are similarly treated, and thus the noncommutative toric geometry of $\mathbb{C P}_{\theta}^{2}=X_{\theta}\left[\Sigma_{\mathbb{C P}^{2}}\right]$ can be assembled into a diagram of gluing morphisms

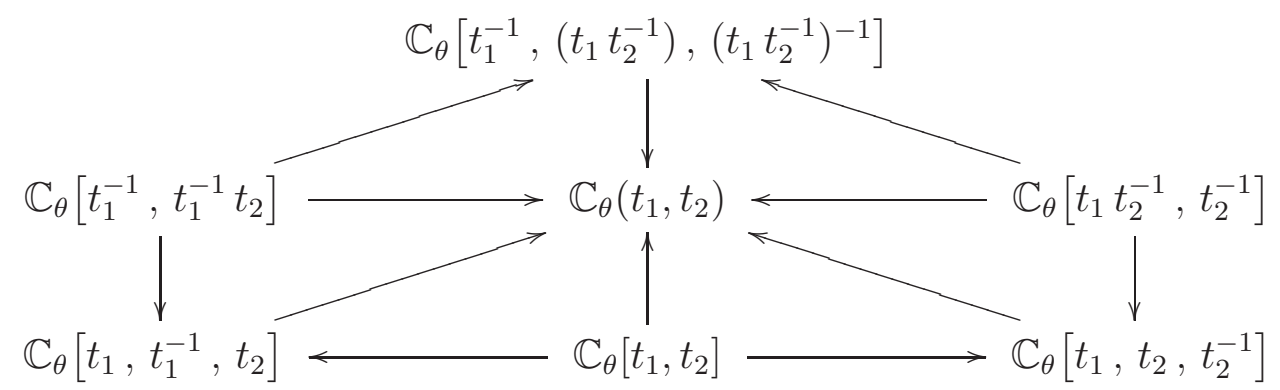

The noncommutative affine variety associated to the zero cone is the spectrum of the full deformed character algebra $\mathbb{C}_{\theta}[0]=\mathbb{C}_{\theta}\left(t_{1}, t_{2}\right)$, corresponding to the open embedding of the noncommutative complex torus into $\mathbb{C P}_{\theta}^{2}$.

This construction generalizes straightforwardly to higher-dimensional projective spaces $\mathbb{C P}^{n}, n>2$, leading to noncommutative projective spaces $\mathbb{C P}_{\theta}^{n}$.

Homogeneous coordinate algebras. We now describe a homogeneous coordinate algebra for the noncommutative projective spaces $\mathbb{C P}_{\theta}^{n}$, recovering a local description by noncommutative Ore localization. For this, consider the embedding $\left(\mathbb{C}_{\theta}^{\times}\right)^{n} \hookrightarrow\left(\mathbb{C}_{\tilde{\theta}}^{\times}\right)^{n+1}$ with

$$
\tilde{\theta}=\left(\begin{array}{ll}
\theta & 0 \\
0 & 0
\end{array}\right)
$$


The corresponding complex Moyal plane $\mathbb{C}_{\tilde{\theta}}^{n+1}$ is defined by the graded polynomial algebra $\mathbb{C}_{\tilde{\theta}}\left[w_{1}, \ldots, w_{n+1}\right]$ in $n+1$ generators $w_{i}, i=1, \ldots, n+1$, of degree 1 with quadratic relations

$$
\begin{aligned}
w_{n+1} w_{i} & =w_{i} w_{n+1}, \quad i=1, \ldots, n, \\
w_{i} w_{j} & =q_{i j}^{2} w_{j} w_{i}, \quad i, j=1, \ldots, n .
\end{aligned}
$$

This graded algebra is called the homogeneous coordinate algebra $\mathcal{A}=\mathcal{A}\left(\mathbb{C P}_{\theta}^{n}\right)$ of the noncommutative variety $\mathbb{C P}_{\theta}^{n}$. The grading on $\mathcal{A}$ is by the usual polynomial degree. There is a natural coaction ${ }_{\mathcal{A}} \Delta: \mathcal{A} \rightarrow \mathcal{A} \otimes \mathcal{F}_{n}^{\theta}$ with ${ }_{\mathcal{A}} \Delta\left(w_{i}\right)=w_{i} \otimes g_{i i}$ for $i=1, \ldots, n$, and ${ }_{\mathcal{A}} \Delta\left(w_{n+1}\right)=w_{n+1} \otimes 1$, defined via the embedding $\left(\mathbb{C}_{\theta}^{\times}\right)^{n} \hookrightarrow\left(\mathbb{C}_{\tilde{\theta}}^{\times}\right)^{n+1}$. This makes $\mathcal{A}$ into an object of the category ${ }^{\mathcal{H}_{\theta}} \mathscr{M}$.

It is straightforward to verify that each monomial $w_{i}$ generates a left (and right) denominator set in $\mathcal{A}$. Let $\mathcal{A}\left[w_{i}^{-1}\right]$ be the left Ore localization of $\mathcal{A}$ with respect to $w_{i}$. Since $w_{i}$ is homogeneous of degree 1 , the algebra $\mathcal{A}\left[w_{i}^{-1}\right]$ is also $\mathbb{N}_{0}$-graded. Elements of degree 0 in $\mathcal{A}\left[w_{i}^{-1}\right]$ form a subalgebra which we denote by $\mathcal{A}\left[w_{i}^{-1}\right]_{0}$. Noncommutative affine subvarieties associated to maximal cones can be recovered as the Ore localizations of the homogeneous coordinate algebra, since for each maximal cone $\sigma_{i}$ there is a natural isomorphism of noncommutative algebras $\mathbb{C}_{\theta}\left[\sigma_{i}\right] \cong \mathcal{A}\left[w_{i}^{-1}\right]_{0}$ in the category ${ }^{\mathcal{H}_{\theta}} \mathscr{M}[5$, Thm. 5.4]. Note, however, that the surjective map $\mathcal{A} \rightarrow \mathcal{A}\left[w_{i}^{-1}\right]_{0}$ is in general an algebra homomorphism only for $i=n+1$ corresponding to the central generator $w_{n+1}$. In the classical case $\theta=0$, the category of coherent sheaves on the projective space $\mathbb{C P}^{n}$ is equivalent to the category of finitely-generated $\mathcal{A}$-modules [1, Cor. 2.4]; in $\S 2.1$ we show that this equivalence extends to the noncommutative case $\theta \neq 0$ as well.

We use noncommutative homogeneous coordinate algebras to introduce noncommutative projective varieties. If $I \subset \mathcal{A}$ is a graded two-sided ideal generated by a set of homogeneous polynomials $f_{1}, \ldots, f_{m} \in \mathbb{C}_{\tilde{\theta}}\left[w_{1}, \ldots, w_{n+1}\right]$, then the quotient algebra $\mathcal{A}_{I}:=\mathcal{A} / I$ is the coordinate algebra of a noncommutative projective variety. The projection $\pi_{I}: \mathcal{A} \rightarrow \mathcal{A}_{I}$ can be regarded as the dual of a closed embedding given by $X_{\theta}(I) \hookrightarrow \mathbb{C P}_{\theta}^{n}$, identified with the common zero locus in $\mathbb{C}_{\tilde{\theta}}^{n+1}$ given by the set of relations $\left\{f_{1}=0, \ldots, f_{m}=0\right\}$. Its homogeneous coordinate algebra $\pi_{I}\left(\mathbb{C}_{\tilde{\theta}}\left[w_{1}, \ldots, w_{n+1}\right]\right)$ has relations $(1.25)$ and $f_{1}=0, \ldots, f_{m}=0$. It is also graded, with $\left(\mathcal{A}_{I}\right)_{0}=\mathbb{C}$ and $\operatorname{dim}_{\mathbb{C}}\left(\mathcal{A}_{I}\right)_{k}<\infty$ for all $k \geq 0$. The $\mathcal{H}_{\theta}$-coaction on $\mathcal{A}$ naturally restricts to $\mathcal{A}_{I}$.

Noncommutative grassmannians and flag varieties. A prominent example of noncommutative projective varieties in the following will be noncommutative Grassmann varieties $\operatorname{Gr}_{\theta}(d ; n)$ associated to an $\mathcal{H}_{\theta}$-comodule $V$ of dimension $n>d$. An element of the homogeneous coordinate algebra of $\mathbb{G r}_{\theta}(d ; n)$ is defined as an element in $\mathbb{P}\left(\bigwedge_{\theta}^{d} V\right)$, obtained by taking the $\theta$-deformed exterior product of $d$ rows of a matrix in $\mathcal{A}\left(\mathrm{GL}_{\theta}(n)\right.$ ) (and quotienting by the appropriate equivalence relation). We take a noncommutative $d \times n$ matrix representing an element of $\mathcal{A}\left(\mathbb{G r}_{\theta}(d ; n)\right)$ and send it into the $\left(\begin{array}{l}n \\ d\end{array}\right)$-tuple of its minors; we label each of them $\Lambda^{J}$ by ordered $d$-multi-indices $J=\left(j_{1} \cdots j_{d}\right), 1 \leq j_{\alpha} \leq n$. Then we provide noncommutative relations between minors, seen now as homogeneous coordinates in $\mathcal{A}\left(\mathbb{C P}_{\Theta}^{N}\right)$ with $N=\left(\begin{array}{l}n \\ d\end{array}\right)-1$, together with noncommutative Plücker relations between them. 
From (1.16) with $|J|=\left|J^{\prime}\right|=d$ representing two different minors we have

$$
\Lambda^{J} \Lambda^{J^{\prime}}=\left(\prod_{\alpha, \beta=1}^{d} q_{j_{\alpha} j_{\beta}^{\prime}}^{2}\right) \Lambda^{J^{\prime}} \Lambda^{J} .
$$

With respect to the noncommutative algebraic torus $T_{\theta}=\left(\mathbb{C}_{\theta}^{\times}\right)^{n}$, the commutation relations (1.26) come from regarding the coordinate algebra $\mathcal{A}\left(\mathbb{G r}_{\theta}(d ; n)\right)$ naturally as an object of the category ${ }^{\mathcal{H}_{\theta}} \mathscr{M}$ by the left coaction

$$
\Delta_{\mathcal{A}\left(\mathbb{G r}_{\theta}(d ; n)\right)}: \mathcal{A}\left(\mathbb{G r}_{\theta}(d ; n)\right) \longrightarrow \mathcal{H}_{\theta} \otimes \mathcal{A}\left(\mathbb{G r}_{\theta}(d ; n)\right)
$$

given on generators by

$$
\Delta_{\mathcal{A}\left(\mathbb{G r}_{\theta}(d ; n)\right)}\left(\Lambda^{J}\right)=t_{J} \otimes \Lambda^{J}, \quad t_{J}:=t_{j_{1}} \cdots t_{j_{d}} .
$$

This implies that the $N \times N$ noncommutativity matrix $\Theta$ of the projective space containing the embedding of $\mathbb{G r}_{\theta}(d ; n)$ is completely determined (mod $\left.2 \pi\right)$ from the $n \times n$ noncommutativity matrix $\theta$ of the grassmannian. Necessary and sufficient conditions for the existence of the embedding of $\mathbb{G r}_{\theta}(d ; n)$ into $\mathcal{A}\left(\mathbb{C P}_{\Theta}^{N}\right)$ are thus

$$
\Theta^{J J^{\prime}}=\sum_{\alpha, \beta=1}^{d} \theta^{j_{\alpha} j_{\beta}^{\prime}}
$$

Noncommutative Plücker relations generate an ideal in the homogeneous coordinate algebra $\mathcal{A}\left(\mathbb{C P}_{\Theta}^{N}\right)$ of the projective space, and we will define the noncommutative quotient algebra to be the homogeneous coordinate algebra $\mathcal{A}\left(\mathbb{G r}_{\theta}(d ; n)\right)$ of the (embedding of the) noncommutative grassmannian. From the noncommutative Laplace expansions (1.14) and (1.15) we see that noncommutative minors of order $d$ in $\mathrm{GL}_{\theta}(n)$ obey Plücker relations

$$
\sum_{\gamma=1}^{d+1} \prod_{\alpha=1}^{d} \prod_{\beta=1}^{d-1}(-1)^{\gamma+1} q_{i_{\gamma} i_{\alpha}^{\gamma}} q_{i_{\gamma} j_{\beta}} \Lambda^{I^{\gamma}} \Lambda^{i_{\gamma} \cup J}=0
$$

for every choice of multi-indices $I$ and $J$ with $|I|=d+1$ and $|J|=d-1$.

The generalization to flag varieties is straightforward. Consider an increasing chain of nested vector subspaces of $V$,

$$
0=V_{0} \varsubsetneqq V_{1} \varsubsetneqq V_{2} \varsubsetneqq \cdots \varsubsetneqq V_{r+1}=V,
$$

such that $\gamma_{i}=\operatorname{dim}_{\mathbb{C}}\left(V_{i}\right)-\operatorname{dim}_{\mathbb{C}}\left(V_{i-1}\right)$ for $i=1, \ldots, r+1$ and $\gamma=\left(\gamma_{1}, \ldots, \gamma_{r+1}\right)$ with $1 \leq r \leq n-1$ is a partition of $n$. The corresponding flag variety $\mathbb{F l}(\gamma ; n)$ is the moduli space of chains (or "flags") associated to the sequence $\gamma=\left(\gamma_{1}, \ldots, \gamma_{r+1}\right)$. The Plücker embedding of flag varieties into projective spaces is similar to the case of grassmannians. Set $d_{i}=\sum_{a \leq i} \gamma_{a}=\operatorname{dim}_{\mathbb{C}}\left(V_{i}\right)$ for $i=1, \ldots, r+1$. A point in $\mathbb{F l}(\gamma ; n)$ is represented by an equivalence class $[A]$ in $\mathrm{GL}(n)$ with respect to the equivalence relation given by the action of block diagonal matrices whose blocks are invertible transformations in each nested subspace $V_{i}$. For each $i$ there is a Plücker map $\mathrm{Pl}_{i}: \mathbb{F l}(\gamma ; n) \rightarrow \mathbb{C P}^{N_{i}}$, with $N_{i}=\left(\begin{array}{c}n \\ d_{i}\end{array}\right)-1$, whose image is the $\left(\begin{array}{c}n \\ d_{i}\end{array}\right)$-tuple of all minors of $A$ obtained from the first $d_{i}$ rows. Hence each minor is labelled by a multi-index representing the $d_{i}$ columns involved while the rows are always given by the standard ordered multi-index $\left(12 \cdots d_{i}\right)$. Assembling all of these maps together we get a Plücker embedding

$$
\mathrm{Pl}: \mathbb{F l}(\gamma ; n) \longrightarrow \mathbb{C P}(\gamma ; n):=\mathbb{C P}^{N_{1}} \times \cdots \times \mathbb{C P}^{N_{r}},
$$

where the last factor for $i=r+1$ gives a trivial contribution since $N_{r+1}=\left(\begin{array}{l}n \\ n\end{array}\right)-1=0$. The image of the Plücker map $\mathrm{Pl}$ in $\mathbb{C P}(\gamma ; n)$ is described by a set of quadratic equations 
called Young symmetry relations. In the noncommutative setting we follow the same idea. In addition to noncommutative Plücker relations (1.29) we now have noncommutative relations (1.16) between minors of different size, i.e. with multi-indices of different lengths $|I|=|J|=d$ and $\left|I^{\prime}\right|=\left|J^{\prime}\right|=d^{\prime}$, and "genuine" noncommutative Young symmetry relations among minors of different order. Again from the Laplace expansion of the minors in (1.14) and (1.15) one shows that noncommutative minors of order $d$ and $d^{\prime}$ in $\mathrm{GL}_{\theta}(n)$ obey Young symmetry relations

$$
\sum_{\gamma=1}^{d+1} \prod_{\mu=1}^{d} \prod_{\nu=1}^{d^{\prime}-1}(-1)^{\gamma+1} q_{i_{\gamma} i_{\mu}^{\gamma}} q_{i_{\gamma} j_{\nu}} \Lambda^{I^{\gamma}} \Lambda^{i_{\gamma} \cup J}=0,
$$

for every choice of multi-indices $I, J$ with $|I|=d+1$ and $|J|=d^{\prime}-1$. The coordinate algebra of the noncommutative flag variety $\mathbb{F} l_{\theta}(\gamma ; n)=\mathbb{F} l_{\theta}\left(d_{1}, \ldots, d_{r} ; n\right)$ is the quotient of the homogeneous coordinate algebra of $\mathbb{C P}_{\Theta}(\gamma ; n)$ by the ideal generated by the noncommutative Young symmetry relations (1.31).

\section{Noncommutative instantons}

In this section we spell out our explicit construction of noncommutative instantons in three related guises: in the algebro-geometric form of framed sheaves on the noncommutative projective plane $\mathbb{C P}_{\theta}^{2}$, in the linear algebraic form of a braided ADHM parametrization, and in the differential geometric form of anti-selfdual connections on a noncommutative four-sphere $S_{\theta}^{4}$. The construction of instanton bundles is based on a noncommutative version of the twistor transform. Via a classification of the real structures on the grassmannian $\mathbb{G r}_{\theta}(2 ; 4)$ we prove here that our particular deformation of $S^{4}$ is in fact the unique noncommutative four-sphere which is compatible with the noncommutative twistor correspondence.

2.1. Sheaves on noncommutative toric varieties. A sheaf theory on noncommutative toric varieties is straightforwardly developed. The idea is that the "topology" of the noncommutative variety $X_{\theta}=X_{\theta}[\Sigma]$ is given by the cones in the fan $\Sigma$; they form the category $\operatorname{Open}\left(X_{\theta}\right)$ of toric open sets. Then $\operatorname{Open}\left(X_{\theta}\right)$ always contains a sufficiently fine open cover, i.e. sets of inclusions $\left(\sigma_{i} \hookrightarrow \sigma\right)_{i \in I}$ of cones of $\Sigma$ such that $\sigma=\bigcup_{i \in I} \sigma_{i}$. The category Open $\left(X_{\theta}\right)$ with the data of coverings forms a Grothendieck topology on $X_{\theta}$.

The assignment $\sigma \mapsto \mathbb{C}_{\theta}[\sigma]$ of the noncommutative algebra $\mathbb{C}_{\theta}[\sigma]$ to every cone $\sigma \in \Sigma$ defines a sheaf of algebras on Open $\left(X_{\theta}\right)$ [5, Prop. 4.2]; it is regarded as the structure sheaf $\mathcal{O}_{X_{\theta}}$ of the noncommutative toric variety $X_{\theta}$. Let $\bmod \left(X_{\theta}\right)$ be the category of sheaves of right $\mathcal{O}_{X_{\theta}}$-modules on $\operatorname{Open}\left(X_{\theta}\right)$. If $\Sigma$ consists of a single cone $\sigma$, i.e. $X_{\theta}[\Sigma]=U_{\theta}[\sigma]$ is an affine variety with coordinate algebra $\mathbb{C}_{\theta}[\sigma]$, then

$$
\bmod \left(U_{\theta}[\sigma]\right) \cong \bmod \left(\mathbb{C}_{\theta}[\sigma]\right)
$$

coincides with the category of right $\mathbb{C}_{\theta}[\sigma]$-modules. We denote by $\widetilde{M}$ the sheaf associated to a module $M$ under the isomorphism (2.1). A sheaf of right $\mathcal{O}_{X_{\theta}}$-modules is coherent if its restriction to each affine open set $U_{\theta}[\sigma]$ is of the form $\widetilde{M}$ for some finitely-generated right $\mathbb{C}_{\theta}[\sigma]$-module $M$. Coherent sheaves of right $\mathcal{O}_{X_{\theta}}$-modules form a category denoted $\operatorname{coh}\left(X_{\theta}\right)$. There are restriction functors

$$
j_{\sigma}^{\bullet}: \operatorname{coh}\left(X_{\theta}\right) \longrightarrow \bmod \left(\mathbb{C}_{\theta}[\sigma]\right)
$$


for each open inclusion $j_{\sigma}: U[\sigma] \hookrightarrow X[\Sigma]$. Let $\operatorname{tor}(\sigma)$ be the full Serre subcategory of $\operatorname{coh}\left(X_{\theta}\right)$ generated by objects $E$ such that $j_{\sigma}^{\bullet}(E)=0$. In [11, Prop. 4.3] it is proven that the restriction functor (2.2) is exact, and there is a natural equivalence of categories

$$
\operatorname{coh}\left(X_{\theta}\right) / \operatorname{tor}(\sigma) \cong \bmod \left(\mathbb{C}_{\theta}[\sigma]\right)
$$

We use (2.3) to reduce geometric problems in the category $\operatorname{coh}\left(X_{\theta}\right)$ to algebraic problems in the algebra $\mathbb{C}_{\theta}[\sigma]$ via the localization functors $j_{\sigma}^{\bullet}$. A coherent sheaf $\mathcal{E} \in \operatorname{coh}\left(X_{\theta}\right)$ is locally free or a bundle if each $\mathcal{E}_{\sigma}:=j_{\sigma}^{\bullet}(\mathcal{E}), \sigma \in \Sigma$, corresponds to a free module $\mathbb{C}_{\theta}[\sigma]^{\oplus r}$ for some integer $r \in \mathbb{N}$ called the rank of $\mathcal{E}$. A coherent sheaf $E \in \operatorname{coh}\left(X_{\theta}\right)$ is torsion free if each $E_{\sigma}, \sigma \in \Sigma$, has no finite-dimensional submodules, or equivalently if it admits an embedding $E \hookrightarrow \mathcal{E}$ into a locally free sheaf $\mathcal{E}$; the rank of $E$ is the rank of $\mathcal{E}$ minus the rank of $\mathcal{E} / E$. For any pair of sheaves $E, F \in \operatorname{coh}\left(X_{\theta}\right)$, let $\operatorname{Ext}^{p}(E, F)$ be the $p$-th derived functor of the functor $\operatorname{Hom}(E, F)=\operatorname{Hom}_{\mathrm{coh}\left(X_{\theta}\right)}(E, F)$. For a sheaf $E \in \operatorname{coh}\left(X_{\theta}\right)$, we define $H^{p}\left(X_{\theta}, E\right):=\operatorname{Ext}^{p}\left(\mathcal{O}_{X_{\theta}}, E\right)$.

We will mostly work on projective varieties. Here many algebraic and geometric properties are inherited from $\mathbb{C P}_{\theta}^{n}$ and its homogeneous coordinate algebra $\mathcal{A}=\mathcal{A}\left(\mathbb{C P}_{\theta}^{n}\right)$ by restriction. The algebra $\mathcal{A}$ has nice "smoothness" properties which enable straightforward extensions of many classical constructs within the realm of noncommutative algebraic geometry. In particular, $\mathcal{A}$ is an Artin-Schelter regular algebra of global homological dimension $n+1$. Let $\operatorname{gr}(\mathcal{A})$ be the category of finitely-generated graded right $\mathcal{A}$-modules $M=\bigoplus_{k \geq 0} M_{k}$ and degree zero morphisms, and let $\operatorname{tor}(\mathcal{A})$ be the full Serre subcategory of $\operatorname{gr}(\mathcal{A})$ consisting of finite-dimensional graded $\mathcal{A}$-modules $M$, i.e. $M_{k}=0$ for $k \gg 0$. From (2.3) it follows that the category of coherent sheaves on Open $\left(\mathbb{C P}_{\theta}^{n}\right)$ can be identified with the abelian quotient category $\operatorname{coh}\left(\mathbb{C P}_{\theta}^{n}\right):=\operatorname{gr}(\mathcal{A}) / \operatorname{tor}(\mathcal{A})$. Let $\pi: \operatorname{gr}(\mathcal{A}) \rightarrow \operatorname{coh}\left(\mathbb{C P}_{\theta}^{n}\right)$ be the canonical projection functor; it has a natural right adjoint functor $\Gamma: \operatorname{coh}\left(\mathbb{C P}_{\theta}^{n}\right) \rightarrow \operatorname{gr}(\mathcal{A})$. Under this correspondence, the structure sheaf $\mathcal{O}_{\mathbb{C P}_{\theta}^{n}}$ is the image $\pi(\mathcal{A})$ of the homogeneous coordinate algebra itself, regarded as a free right $\mathcal{A}$-module of rank one. If $E=\pi(M)$ where $M \in \operatorname{gr}(\mathcal{A})$ is a graded right $\mathcal{A}$-module, then $M\left[w_{i}^{-1}\right]_{0}=\left(M \otimes_{\mathcal{A}} \mathcal{A}\left[w_{i}^{-1}\right]\right)_{0}$ is a right $\mathbb{C}_{\theta}\left[\sigma_{i}\right]$-module for each $i=1, \ldots, n+1$. For $k \in \mathbb{Z}$, we let $E(k)$ be the sheaf corresponding to the module $M$ with its grading shifted by $k$ units.

Tautological bundle on $\mathbb{G r}_{\theta}(d ; n)$. An important example of a locally free sheaf in the following is the tautological bundle $\mathcal{S}_{\theta}$ on $\operatorname{Open}\left(\mathbb{G r}_{\theta}(d ; n)\right)$, which further admits a straightforward extension to noncommutative flag varieties. The bundle $\mathcal{S}_{\theta}$ is the subsheaf of elements of the free module $\left(f_{1}(\Lambda), \ldots, f_{n}(\Lambda)\right) \in \mathcal{A}\left(\mathbb{G r}_{\theta}(d ; n)\right)^{\oplus n}$ over the noncommutative grassmannian which satisfy the equations

$$
\sum_{\alpha=1}^{d+1}(-1)^{\alpha}\left(\prod_{\beta=1}^{d} q_{j_{\alpha} j_{\beta}^{\alpha}}\right) \Lambda^{J \backslash j_{\alpha}} f_{j_{\alpha}}(\Lambda)=0
$$

for every ordered $(d+1)$-multi-index $J=\left(j_{1} \cdots j_{d+1}\right)$ with $j_{1}<j_{2}<\cdots<j_{d+1}$, where the minors of order $d$ obey the relations (1.26). The "geometric" interpretation of $(2.4)$ is that we add the vector $f(\Lambda)=\left(f_{1}(\Lambda), \ldots, f_{n}(\Lambda)\right)$ to the $d \times n$ matrix $\Lambda$ and require that all the minors, expanded along the $(d+1)$-th row $f(\Lambda)$ via the noncommutative Laplace expansion (1.14), are zero. This is equivalent to saying that the vector $f(\Lambda)$ belongs to the $d$-plane $\Lambda$, the condition which characterizes $\mathcal{S}_{\theta}$ inside the trivial bundle $\mathcal{A}\left(\mathbb{G r}_{\theta}(d ; n)\right)^{\oplus n}$. It is proven in $[5$, Prop. 6.15$]$ that the sheaf $\mathcal{S}_{\theta}$ is indeed locally free on Open $\left(\mathbb{G r}_{\theta}(d ; n)\right)$. 
We can use the Plücker map to regard the noncommutative minors $\Lambda^{J \backslash j_{\alpha}}$ as homogeneous coordinates in $\mathbb{P}\left(\bigwedge_{\theta}^{d} V\right)$. In this case we have to consider the restriction of $(2.4)$ to those elements $\Lambda^{J}$ which also satisfy the Young symmetry relations (1.29). This gives the sheaf $\mathcal{S}_{\theta}$ the natural structure of a graded $\mathcal{A}\left(\mathbb{G r}_{\theta}(d ; n)\right)$-bimodule.

2.2. Twistor geometry. Consider the explicit algebraic relations for the noncommutative Plücker embedding of the Klein quadric $\mathbb{G r}_{\theta}(2 ; 4)$ in $\mathbb{C P}_{\Theta}^{5}$. The algebra $\mathcal{A}\left(\mathbb{G r}_{\theta}(2 ; 4)\right)$ has six generators $\Lambda^{(i j)}, 1 \leq i<j \leq 4$; we give to the multi-indices labelling the minors a lexicographic ordering such that $1=(12), 2=(13), 3=(14), 4=(23), 5=(24)$, and $6=(34)$. Then the expression (1.28) for the skew-symmetric noncommutativity matrix $\Theta$ in terms of entries of $\theta$ is given by

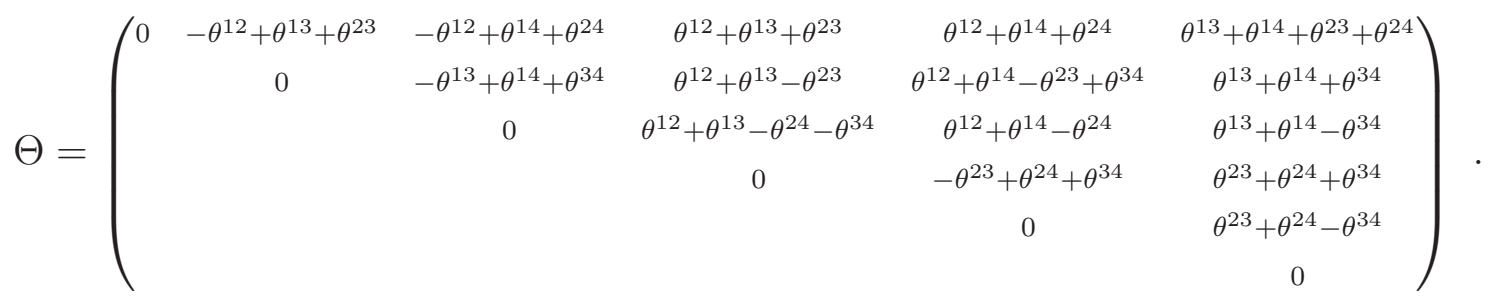

When considering noncommutative Plücker relations (1.29), various choices for the multiindices $I$ and $J$ give two-term equations, which yield known noncommutative relations among minors. The only three-term Plücker equation, obtained from $I=(123)$ and $J=(4)$, is a noncommutative deformation of the well-known classical equation describing the Klein quadric $\mathbb{G r}(2 ; 4) \hookrightarrow \mathbb{C P}^{5}$. After rearranging all indices labelling the minors in increasing order using antisymmetry, and the minors themselves using (1.26), we obtain

$$
q_{31} q_{32} q_{34} \Lambda^{(12)} \Lambda^{(34)}-q_{21} q_{23} q_{24} \Lambda^{(13)} \Lambda^{(24)}+q_{12} q_{13} q_{14} \Lambda^{(23)} \Lambda^{(14)}=0 .
$$

We will describe the homogeneous coordinate algebra of the noncommutative partial flag variety $\mathbb{F} l_{\theta}(1,2 ; 4)$ using the algebra projection $[5]$

$$
\mathcal{A}\left(\mathbb{C P}_{\theta}^{3}\right) \otimes_{\theta} \mathcal{A}\left(\mathbb{G r}_{\theta}(2 ; 4)\right) \longrightarrow \mathcal{A}\left(\mathbb{F l} l_{\theta}(1,2 ; 4)\right) .
$$

The braided tensor product algebra $\mathcal{A}\left(\mathbb{C P}_{\theta}^{3}\right) \otimes_{\theta} \mathcal{A}\left(\mathbb{G r}_{\theta}(2 ; 4)\right)$, as an object in the category $\mathcal{H}_{\theta} \mathscr{M}$, is generated by the homogeneous coordinate elements $w_{i}$ with relations (1.26) for $d=1$, i.e. $w_{i} w_{j}=q_{i j}^{2} w_{j} w_{i}$, for $i, j=1,2,3,4$, and by the noncommutative $2 \times 2$ minors $\Lambda^{\left(j_{1} j_{2}\right)}, 1 \leq j_{1}<j_{2} \leq 4$, obeying (1.26) for $d=2$ and the quadric relation (2.5). They also obey the structure equations (relations (1.16) among minors of different order)

$$
w_{i} \Lambda^{\left(j_{1} j_{2}\right)}=q_{12}^{-2} q_{i j_{1}}^{2} q_{i j_{2}}^{2} \Lambda^{\left(j_{1} j_{2}\right)} w_{i}
$$

for all $i=1,2,3,4$ and $1 \leq j_{1}<j_{2} \leq 4$. The remaining noncommutative Plücker equations come from the Young symmetry relations (1.31) with $d=2$ and $d^{\prime}=1$, and one finds the additional relations

$$
\begin{aligned}
& q_{12} q_{13} \Lambda^{(23)} w_{1}-q_{21} q_{23} \Lambda^{(13)} w_{2}+q_{31} q_{32} \Lambda^{(12)} w_{3}=0, \\
& q_{12} q_{14} \Lambda^{(24)} w_{1}-q_{21} q_{24} \Lambda^{(14)} w_{2}+q_{41} q_{42} \Lambda^{(12)} w_{4}=0, \\
& q_{13} q_{14} \Lambda^{(34)} w_{1}-q_{31} q_{34} \Lambda^{(14)} w_{3}+q_{41} q_{43} \Lambda^{(13)} w_{4}=0, \\
& q_{23} q_{24} \Lambda^{(34)} w_{2}-q_{32} q_{34} \Lambda^{(24)} w_{3}+q_{42} q_{43} \Lambda^{(23)} w_{4}=0 .
\end{aligned}
$$


Consider now the noncommutative correspondence diagram

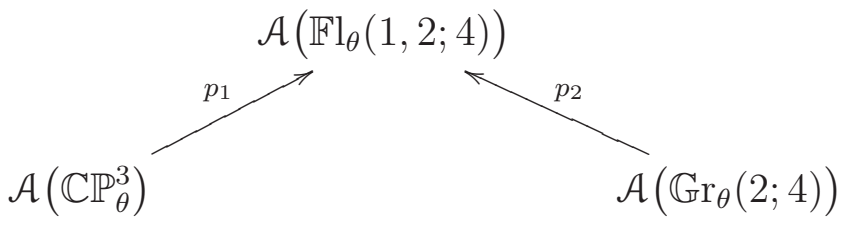

which is a noncommutative deformation of the usual Penrose twistor correspondence, with $\mathcal{A}\left(\mathbb{C P}_{\theta}^{3}\right)$ the "noncommutative twistor algebra". The morphism $E \mapsto p_{2}{ }^{*} p_{1 *}(E)$ gives a map from sheaves in $\operatorname{coh}\left(\mathbb{C P}_{\theta}^{3}\right)$ to sheaves in $\operatorname{coh}\left(\mathbb{G r}_{\theta}(2 ; 4)\right)$, yielding a noncommutative deformation of the usual Penrose-Ward twistor transform. Given a graded right $\mathcal{A}\left(\mathbb{C P}_{\theta}^{3}\right)$ module $M$ in $\operatorname{gr}\left(\mathcal{A}\left(\mathbb{C P}_{\theta}^{3}\right)\right)$, the push-forward

$$
M^{\prime}=p_{1 *}(M)=M \otimes_{\mathcal{A}\left(\mathbb{C P}_{\theta}^{3}\right)} \mathcal{A}\left(\mathbb{F l}_{\theta}(1,2 ; 4)\right)
$$

is a bigraded right module over $\mathcal{A}\left(\mathbb{F} l_{\theta}(1,2 ; 4)\right)$, where on the right-hand side we regard the algebra $\mathcal{A}\left(\mathbb{F l}_{\theta}(1,2 ; 4)\right)$ as an $\mathcal{A}\left(\mathbb{C P}_{\theta}^{3}\right)$-bimodule. The diagonal subspace of this module, in the sense of $[12, \S 8]$, induces the push-forward functor $p_{1 *}: \operatorname{coh}\left(\mathbb{C P}_{\theta}^{3}\right) \rightarrow \operatorname{coh}\left(\mathbb{F} l_{\theta}(1,2 ; 4)\right)$. Similarly, one defines the push-forward functor $p_{2 *}: \operatorname{coh}\left(\mathbb{G r}_{\theta}(2 ; 4)\right) \rightarrow \operatorname{coh}\left(\mathbb{F} l_{\theta}(1,2 ; 4)\right)$, which has a right adjoint functor $p_{2}{ }^{*}: \operatorname{coh}\left(\mathbb{F} l_{\theta}(1,2 ; 4)\right) \rightarrow \operatorname{coh}\left(\mathbb{G r}_{\theta}(2 ; 4)\right)$. In [6, Lem. 3.14] the noncommutative twistor transforms of the elementary bundles $\mathcal{O}_{\mathbb{C P}_{\theta}^{3}}(k)$ for $k \in \mathbb{Z}$ are computed to be

$$
p_{2}{ }^{*} p_{1 *}\left(\mathcal{O}_{\mathbb{C P}_{\theta}^{3}}(k)\right)=\left\{\begin{array}{rl}
\operatorname{Sym}_{\theta}^{k}\left(\mathcal{S}_{\theta}\right) & , \quad k \geq 0 \\
0, & k<0
\end{array},\right.
$$

where $\mathcal{S}_{\theta}$ is the tautological bundle on $\operatorname{Open}\left(\mathbb{G r}_{\theta}(2 ; 4)\right)$ and $\operatorname{Sym}_{\theta}^{k}\left(\mathcal{S}_{\theta}\right)$ is the bundle associated to the graded right $\mathcal{A}\left(\mathbb{G r}_{\theta}(2 ; 4)\right)$-module

$$
\Gamma\left(\mathcal{S}_{\theta}\right)^{\otimes k} /\left\langle s_{1} \otimes s_{2}-\Psi_{\Gamma\left(\mathcal{S}_{\theta}\right), \Gamma\left(\mathcal{S}_{\theta}\right)}^{\theta}\left(s_{1} \otimes s_{2}\right)\right\rangle_{s_{1}, s_{2} \in \Gamma\left(\delta_{\theta}\right)} .
$$

2.3. Real structures on the noncommutative Klein quadric. In order to introduce a *-algebra structure on $\mathcal{A}\left(\mathbb{G r}_{\theta}(2 ; 4)\right)$ and a compatible twistor construction of instanton bundles later on, from now on we set

$$
q_{12}=q_{21}^{-1}=: q, \quad q_{i j}=1 \quad \text { otherwise . }
$$

From the perspective of the Drinfel'd twist deformation, this choice restricts the $\mathcal{H}_{\theta^{-}}$ coaction to a coaction of the subalgebra $\mathbb{C}_{\theta}\left(t_{1}, t_{2}\right)$ defined by setting $t_{3}=t_{4}=1$, i.e. the underlying noncommutative complex torus $T_{\theta}=\left(\mathbb{C}_{\theta}^{\times}\right)^{4}$ is reduced to $\left(\mathbb{C}_{\theta}^{\times}\right)^{2}$. This entails in particular that the generator $\Lambda^{(34)}$ is central. We classify all possible real structures on $\mathbb{G r}_{\theta}(2 ; 4)$ and determine the induced signature on the Plücker relation (2.5), which for the present choice becomes

$$
q \Lambda^{(12)} \Lambda^{(34)}-\Lambda^{(13)} \Lambda^{(24)}+\Lambda^{(14)} \Lambda^{(23)}=0 .
$$

A real structure $\dagger: \mathcal{A}\left(\mathbb{G r}_{\theta}(2 ; 4)\right) \rightarrow \mathcal{A}\left(\mathbb{G r}_{\theta}(2 ; 4)\right)$ is defined on the generators $\Lambda^{(i j)}$ and then extended to the whole of $\mathcal{A}\left(\mathbb{G r}_{\theta}(2 ; 4)\right)$ as a conjugate linear anti-homomorphism; it must therefore be compatible with the commutation relations (1.26) and the Plücker equation (2.10). By imposing compatibility of $\dagger$ with (1.26), we can easily determine how $\Lambda^{(i j) \dagger}$ should transform under the coaction (1.27). In particular, $\Lambda^{(34) \dagger}$ must be coinvariant, i.e. it is central and hence proportional to $\Lambda^{(34)}$, while $\Lambda^{(12) \dagger}$ transforms with weight $t_{1} t_{2}$ and hence is proportional to $\Lambda^{(12)}$. We then write

$$
\Lambda^{(12) \dagger}=\mu \Lambda^{(12)}, \quad \Lambda^{(34) \dagger}=\nu \Lambda^{(34)} \quad \text { for } \quad \mu, \nu \in S^{1}=\{\zeta \in \mathbb{C} \mid \zeta \bar{\zeta}=1\} .
$$


Similarly, $\Lambda^{(13) \dagger}$ and $\Lambda^{(14) \dagger}$ must transform either both with weight $t_{1}$ or both with weight $t_{2}$, and $\Lambda^{(23) \dagger}$ and $\Lambda^{(24) \dagger}$ accordingly both with weight $t_{2}$ or both with weight $t_{1}$.

Suppose first that $\Lambda^{(13) \dagger}$ and $\Lambda^{(14) \dagger}$ transform with weight $t_{1}$; then $q \in S^{1}$ in order to ensure $\left(q \Lambda^{(i j)}\right)^{\dagger}=q^{-1} \Lambda^{(i j) \dagger}$, and a priori $\Lambda^{(13) \dagger}$ and $\Lambda^{(14) \dagger}$ are each linear combinations of both $\Lambda^{(13)}$ and $\Lambda^{(14)}$. Via a basis change automorphism of the generators $\left(\Lambda^{(13)}, \Lambda^{(14)}\right)$ if necessary, we can assume that both $\Lambda^{(13) \dagger}$ and $\Lambda^{(14) \dagger}$ are proportional to single generators without loss of generality. The GL(2) equivalence classes of real structures on $\left\{\Lambda^{(13)}, \Lambda^{(14)}\right\}$ are therefore exhausted by the representatives

$$
\Lambda^{(13) \dagger}=\alpha \Lambda^{(13)}, \quad \Lambda^{(14) \dagger}=\beta \Lambda^{(14)} \quad \text { for } \quad \alpha, \beta \in S^{1}
$$

and

$$
\Lambda^{(13) \dagger}=\alpha \Lambda^{(14)}, \quad \Lambda^{(14) \dagger}=\bar{\alpha}^{-1} \Lambda^{(13)} \quad \text { for } \quad \alpha \in \mathbb{C}
$$

Suppose now that $\Lambda^{(13) \dagger}$ and $\Lambda^{(14) \dagger}$ transform with weight $t_{2}$; this requires $q \in \mathbb{R}$. A similar argument as in the previous case shows that up to basis change automorphism one can choose two classes of real structures with representatives

$$
\Lambda^{(13) \dagger}=\alpha \Lambda^{(23)}, \quad \Lambda^{(14) \dagger}=\beta \Lambda^{(24)} \quad \text { for } \quad \alpha, \beta \in \mathbb{C}
$$

and

$$
\Lambda^{(13) \dagger}=\alpha \Lambda^{(24)}, \quad \Lambda^{(14) \dagger}=\beta \Lambda^{(23)} \quad \text { for } \quad \alpha, \beta \in \mathbb{C}
$$

We now impose compatibility with the Plücker equation (2.10). Since this is the only quadratic relation in $\mathcal{A}\left(\mathbb{G r}_{\theta}(2 ; 4)\right)$, its image under $\dagger$ must be proportional to itself; this requirement also determines in $(2.12)-(2.13)$ the real structure on the generators $\Lambda^{(23)}$ and $\Lambda^{(24)}$. There are four cases in total, one each coming from (2.12)-(2.15).

Let us first consider the choice of real structure (2.12). Compatibility with the Plücker equation requires

$$
\Lambda^{(23) \dagger}=\gamma \Lambda^{(23)}, \quad \Lambda^{(24) \dagger}=\delta \Lambda^{(24)} \quad \text { for } \quad \gamma, \delta \in S^{1} .
$$

Remembering (2.11) and that in this case $q \in S^{1}$, by applying $\dagger$ to (2.10) we find the constraint

$$
\alpha \delta=\beta \gamma=\mu \nu .
$$

To determine the signature of the quadratic form appearing in the Plücker relation, we rewrite (2.10) as

$$
\begin{aligned}
q \bar{\nu} \Lambda^{(12)} \Lambda^{(34) \dagger}+q \bar{\mu} \Lambda^{(34)} \Lambda^{(12) \dagger}-\bar{\delta} \Lambda^{(13)} \Lambda^{(24) \dagger} & \\
& -q^{2} \bar{\alpha} \Lambda^{(24)} \Lambda^{(13) \dagger}+\bar{\gamma} \Lambda^{(14)} \Lambda^{(23) \dagger}+q^{2} \bar{\beta} \Lambda^{(23)} \Lambda^{(14) \dagger}=0 .
\end{aligned}
$$

Requesting that the terms are pairwise real, together with (2.16), amounts to setting

$$
\mu \nu=q^{2}, \quad \alpha= \pm 1, \quad \delta= \pm q^{2}, \quad \beta= \pm 1, \quad \gamma= \pm q^{2} .
$$

A set of generators which diagonalizes the Plücker bilinear form is therefore

$$
X_{ \pm}=q^{-1} \mu \Lambda^{(12)} \pm \Lambda^{(34)}, \quad V_{ \pm}=\bar{\delta} \Lambda^{(13)} \pm \Lambda^{(24)}, \quad W_{ \pm}=\bar{\gamma} \Lambda^{(14)} \pm \Lambda^{(23)}
$$

giving

$$
X_{+} X_{+}^{\dagger}-X_{-} X_{-}^{\dagger}-V_{+} V_{+}^{\dagger}+V_{-} V_{-}^{\dagger}+W_{+} W_{+}^{\dagger}-W_{-} W_{-}^{\dagger}=0
$$


which exhibits signature $(3,3)$. A completely analogous analysis shows that the choices (2.13) and (2.14) also yield a diagonal Plücker equation with signature $(3,3)$, with compatibility conditions $\Lambda^{(23) \dagger}=-q^{2} \bar{\alpha} \Lambda^{(24)}$ and $\mu=q^{2} \bar{\nu}$ in the case (2.13), while $\nu=\bar{\mu}$ and $\beta=-\bar{\alpha}$ for $(2.14)$.

Let us finally consider the choice (2.15). In this case $q$ is real, and the image of the Plücker equation under $\dagger$ imposes the constraint $\bar{\alpha}^{-1} \alpha=\mu \nu=\bar{\beta}^{-1} \beta$. We rewrite (2.10) in the form

$$
\begin{aligned}
q \bar{\nu} \Lambda^{(12)} \Lambda^{(34) \dagger}+q \bar{\mu} \Lambda^{(12) \dagger} \Lambda^{(34)} & -\alpha^{-1} \Lambda^{(13)} \Lambda^{(13) \dagger} \\
& \quad-\bar{\alpha} \Lambda^{(24) \dagger} \Lambda^{(24)}+\bar{\beta} \Lambda^{(23) \dagger} \Lambda^{(23)}+\beta^{-1} \Lambda^{(14)} \Lambda^{(14) \dagger}=0
\end{aligned}
$$

so that asking for the terms to be pairwise real we get in addition $\nu=\bar{\mu}$ and $\alpha, \beta \in \mathbb{R}$. We can diagonalize the first two terms by introducing the combinations $X_{ \pm}=q \mu \Lambda^{(12)} \pm \Lambda^{(34)}$ giving $X_{+} X_{+}^{\dagger}-X_{-} X_{-}^{\dagger}$; the remaining terms are already in diagonal form. The global signature is therefore $(3,3)$ if $\alpha \beta>0$, and $(5,1)$ if $\alpha \beta<0$.

2.4. Noncommutative four-sphere. We construct a noncommutative four-sphere by picking a compatible $*$-involution on the algebra $\mathcal{A}\left(\mathbb{G r}_{\theta}(2 ; 4)\right)$ for which the quadratic form in the Plücker equation (2.10) has signature $(5,1)$. As we have just shown this requires $q \in \mathbb{R}$ and there is a unique family of such real structures parametrized as

$$
\begin{array}{ll}
\Lambda^{(12) \dagger}=\mu \Lambda^{(12)}, & \Lambda^{(34) \dagger}=\bar{\mu} \Lambda^{(34)}, \\
\Lambda^{(13) \dagger}=\alpha \Lambda^{(24)}, & \Lambda^{(14) \dagger}=\beta \Lambda^{(23)} \quad \text { for } \quad \mu \in S^{1}, \alpha, \beta \in \mathbb{R}, \alpha \beta<0 .
\end{array}
$$

The parameters $\mu, \alpha, \beta$ may be absorbed via a suitable redefinition of the generators $\Lambda^{(i j)}$, so that up to change of basis automorphism in (2.17) there is only one real structure with $\mu=\alpha=-\beta=1$. In what follows, however, it will be convenient to make the choice

$$
\mu=1, \quad \alpha=q, \quad \beta=-q^{-1}
$$

for the real structure, so that

$$
\Lambda^{(12) \dagger}=\Lambda^{(12)}, \quad \Lambda^{(34) \dagger}=\Lambda^{(34)}, \quad \Lambda^{(13) \dagger}=q \Lambda^{(24)}, \quad \Lambda^{(14) \dagger}=-q^{-1} \Lambda^{(23)} .
$$

The real structure is not compatible with the whole toric symmetry $\mathcal{H}$. Compatibility forces us to restrict to a coaction coming from a real abelian subgroup $G$ of $T$ endowed with a $*$-structure defined by $\left(t_{1}\right)^{*}=t_{2}$. This subgroup is a copy of $\left(\mathbb{R}^{\times}\right)^{2}$ whose unital *-algebra of coordinate functions we shall denote by $\mathcal{G}=\mathcal{A}(G)=\mathcal{A}\left(\left(\mathbb{R}^{\times}\right)^{2}\right)$. When restricted to $\mathcal{G}$ the twist (1.6) becomes real (remembering that the deformation parameter $q$ is now real), and everything is consistent within the framework of real Drinfel'd twists and associated deformations of $*$-algebras. Thus the algebra $\mathcal{A}\left(\mathbb{G r}_{\theta}(2 ; 4)\right)$ is a comodule *-algebra (only) for $\mathcal{G}$.

When substituted into the Klein quadric equation (2.10) we obtain

$$
q \Lambda^{(12)} \Lambda^{(34)}-q^{-1} \Lambda^{(13)} \Lambda^{(13) \dagger}-q \Lambda^{(14)} \Lambda^{(14) \dagger}=0 .
$$

In the classical case $q=1$, this relation would read as the equation of a four-sphere in a real slice of $\mathbb{C P}^{5}$. We thus interpret the $*$-algebra corresponding to (2.19) as the coordinate algebra $\mathcal{A}\left(S_{\theta}^{4}\right)$ of a noncommutative four-sphere $S_{\theta}^{4}$ with a non-central "radius". For this, let us redefine the generators by writing $q\left(\Lambda^{(12)}-\Lambda^{(34)}\right)=: 2 X$ and $q\left(\Lambda^{(12)}+\Lambda^{(34)}\right)=: 2 R$, where the hermitean elements $R$ and $X$ commute with each other but not with the remaining minors. Simple algebra then transforms the relation (2.19) into

$$
\Lambda^{(13)} \Lambda^{(13) \dagger}+q^{2} \Lambda^{(14)} \Lambda^{(14) \dagger}+X^{2}=R^{2} .
$$


This is a deformation of the equation of a four-sphere in homogeneous coordinates in a real slice of $\mathbb{C P}^{5}$, with a non-central radius $R$. As a consequence, one is not allowed to fix $R$ to some real number (typically $R=1$ in the classical case). Furthermore, the homogeneous element $R$ does not generate a right or left denominator set, as the corresponding fraction elements do not close to an algebra. Thus it is not possible to apply Ore localization with respect to $R$, an operation that classically would correspond to rescaling the homogeneous coordinates to get an affine description of the sphere. Hence for our noncommutative sphere there is no hope for any global affine description. However, one can still use localization to describe local patches for the sphere; using the two hermitean generators $\Lambda^{(12)}$ and $\Lambda^{(34)}$ we can define two localizations to affine subvarieties of the grassmannian $\mathbb{G r}_{\theta}(2 ; 4)$, whose "real slices" are interpreted as local patches of the sphere $S_{\theta}^{4}$. The two generators $\Lambda^{(12)}$ and $\Lambda^{(34)}$ are rather different in nature, and hence so are the corresponding localizations, as $\Lambda^{(34)}$ is central while $\Lambda^{(12)}$ is not.

The generators of the degree zero subalgebra of $\mathcal{A}\left(\mathbb{G r}_{\theta}(2 ; 4)\right)\left[\Lambda^{(34)-1}\right]$ are

$$
\begin{array}{rll}
\xi_{1}=-\Lambda^{(14)} \Lambda^{(34)-1} & , & \xi_{2}=-\Lambda^{(24)} \Lambda^{(34)-1} \\
\bar{\xi}_{1}=\Lambda^{(23)} \Lambda^{(34)-1} & , & \bar{\xi}_{2}=\Lambda^{(13)} \Lambda^{(34)-1}
\end{array}
$$

together with $\rho=q \Lambda^{(12)} \Lambda^{(34)-1}$. The Plücker relation (2.19) becomes $\rho=\xi_{1} \bar{\xi}_{1}-\bar{\xi}_{2} \xi_{2}$ so that the generator $\rho$ is redundant. The localized algebra $\mathcal{A}\left(\mathbb{G r}_{\theta}(2 ; 4)\right)\left[\Lambda^{(34)-1}\right]_{0}$ is then isomorphic to the algebra generated by elements $\xi_{i}, \bar{\xi}_{i}, i=1,2$ with the relations

$$
\begin{aligned}
\xi_{1} \bar{\xi}_{1}=q^{2} \bar{\xi}_{1} \xi_{1} & , & \xi_{2} \bar{\xi}_{2}=q^{-2} \bar{\xi}_{2} \xi_{2}, \\
\xi_{1} \xi_{2}=q^{2} \xi_{2} \xi_{1} & , & \bar{\xi}_{1} \bar{\xi}_{2}=q^{-2} \bar{\xi}_{2} \bar{\xi}_{1}, \\
\xi_{1} \bar{\xi}_{2}=\bar{\xi}_{2} \xi_{1} & , & \xi_{2} \bar{\xi}_{1}=\bar{\xi}_{1} \xi_{2} .
\end{aligned}
$$

This localized algebra may be thought of as dual to a noncommutative variety $\mathbb{C}_{\theta}^{4}$ regarded as an open affine subvariety of the noncommutative Klein quadric $\mathbb{G r}_{\theta}(2 ; 4)$. It will be important later on to note that this deformation can be regarded as originating, via a Drinfel'd twist procedure, from the action of the torus $T=\left(\mathbb{C}^{\times}\right)^{2}$ on $\mathbb{C}^{4}$ giving a left coaction $\Delta_{\mathcal{A}\left(\mathbb{C}_{\theta}^{4}\right)}: \mathcal{A}\left(\underline{\mathbb{C}_{\theta}^{4}}\right) \rightarrow \mathcal{H}_{\theta} \otimes \mathcal{A}\left(\underline{\mathbb{C}_{\theta}^{4}}\right)$ with

$$
\begin{array}{ll}
\Delta_{\mathcal{A}\left(\underline{\mathbb{C}_{\theta}^{4}}\right)}\left(\xi_{1}\right)=t_{1} \otimes \xi_{1} \quad & \Delta_{\mathcal{A}\left(\underline{\mathbb{C}_{\theta}^{4}}\right)}\left(\xi_{2}\right)=t_{2} \otimes \xi_{2}, \\
\Delta_{\mathcal{A}\left(\underline{\mathbb{C}_{\theta}^{4}}\right)}\left(\bar{\xi}_{1}\right)=t_{2} \otimes \bar{\xi}_{1} \quad, & \Delta_{\mathcal{A}\left(\underline{\mathbb{C}_{\theta}^{4}}\right)}\left(\bar{\xi}_{2}\right)=t_{1} \otimes \bar{\xi}_{2},
\end{array}
$$

and extended as an algebra map; in particular $\Delta_{\mathcal{A}\left(\underline{\mathbb{C}_{\theta}^{4}}\right)}(\rho)=\left(t_{1} t_{2}\right) \otimes \rho$.

The $*$-involution of (2.18) gives rise on the generators $\xi_{i}, \bar{\xi}_{i}, i=1,2$ to the relations

$$
\xi_{1}^{\dagger}=q^{-1} \bar{\xi}_{1}, \quad \xi_{2}^{\dagger}=-q^{-1} \bar{\xi}_{2},
$$

from which it also follows that $\rho=q \xi_{1} \xi_{1}^{\dagger}+q^{3} \xi_{2} \xi_{2}^{\dagger}$. The corresponding $*$-algebra is dual to a noncommutative real variety $\mathbb{R}_{\theta}^{4}$, regarded as an open affine subvariety of the noncommutative four-sphere $S_{\theta}^{4}$. Arguing as we did after (2.18), the coordinate algebra $\mathcal{A}\left(\mathbb{R}_{\theta}^{4}\right)$ is a comodule $*$-algebra only for the coaction of $\mathcal{G}=\mathcal{A}\left(\left(\mathbb{R}^{\times}\right)^{2}\right)$, not for the whole $\mathcal{H}=\mathcal{A}(T)$.

A second open affine subvariety of $S_{\theta}^{4}$ is obtained by localization onto another affine subvariety of the grassmannian $\mathbb{G r}_{\theta}(2 ; 4)$ via the non-central hermitean minor $\Lambda^{(12)}$, which 
generates a left denominator set in $\mathcal{A}\left(\mathbb{G r}_{\theta}(2 ; 4)\right)$. The generators of the degree zero subalgebra of $\left[\Lambda^{(12)-1}\right] \mathcal{A}\left(\mathbb{G r}_{\theta}(2 ; 4)\right)$ are

$$
\begin{array}{lll}
\zeta_{1}=-\Lambda^{(12)-1} \Lambda^{(14)} & , & \zeta_{2}=-\Lambda^{(12)-1} \Lambda^{(24)} \\
\bar{\zeta}_{1}=\Lambda^{(12)-1} \Lambda^{(23)} & , & \bar{\zeta}_{2}=\Lambda^{(12)-1} \Lambda^{(13)}
\end{array}
$$

together with $\tilde{\rho}=q \Lambda^{(12)-1} \Lambda^{(34)}$. The Plücker relation (2.19) becomes $\tilde{\rho}=\bar{\zeta}_{1} \zeta_{1}-\zeta_{2} \bar{\zeta}_{2}$ showing that the generator $\tilde{\rho}$ is redundant. Using the multiplication rules for noncommutative Ore localization in $\left[\Lambda^{(12)-1}\right] \mathcal{A}\left(\mathbb{G r}_{\theta}(2 ; 4)\right)_{0}$ one finds an algebra isomorphic to the algebra generated by elements $\zeta_{i}, \bar{\zeta}_{i}, i=1,2$ with the relations

$$
\begin{aligned}
\zeta_{1} \bar{\zeta}_{1}=q^{-2} \bar{\zeta}_{1} \zeta_{1} & , & \zeta_{2} \bar{\zeta}_{2} & =q^{2} \bar{\zeta}_{2} \zeta_{2}, \\
\zeta_{1} \zeta_{2}=q^{-2} \zeta_{2} \zeta_{1} & & \bar{\zeta}_{1} \bar{\zeta}_{2} & =q^{2} \bar{\zeta}_{2} \bar{\zeta}_{1}, \\
\zeta_{1} \bar{\zeta}_{2}=\bar{\zeta}_{2} \zeta_{1} & & \zeta_{2} \bar{\zeta}_{1} & =\bar{\zeta}_{1} \zeta_{2} .
\end{aligned}
$$

The corresponding noncommutative complex variety is denoted $\widetilde{\mathbb{C}}_{\theta}^{4}$. The counterpart of the coaction $(2.21)$ is given by the dual left coaction $\left.\Delta_{\mathcal{A}\left(\underline{\widetilde{\mathbb{C}}_{\theta}^{4}}\right)}: \overline{\mathcal{A}\left(\widetilde{\mathbb{C}}_{\theta}^{4}\right.}\right) \rightarrow \mathcal{H}_{\theta} \otimes \mathcal{A}\left(\underline{\widetilde{\mathbb{C}}_{\theta}^{4}}\right)$ with

$$
\begin{aligned}
& \Delta_{\mathcal{A}\left(\underline{\widetilde{\mathbb{C}}_{\theta}^{4}}\right)}\left(\zeta_{1}\right)=t_{2}^{-1} \otimes \zeta_{1} \quad, \quad \Delta_{\mathcal{A}\left(\underline{\widetilde{\mathbb{C}}_{\theta}^{4}}\right)}\left(\zeta_{2}\right)=t_{1}^{-1} \otimes \zeta_{2}, \\
& \Delta_{\mathcal{A}\left(\underline{\widetilde{\mathbb{C}}_{\theta}^{4}}\right)}\left(\bar{\zeta}_{1}\right)=t_{1}^{-1} \otimes \bar{\zeta}_{1} \quad, \quad \Delta_{\mathcal{A}\left(\underline{\widetilde{\mathbb{C}}_{\theta}^{4}}\right)}\left(\bar{\zeta}_{2}\right)=t_{2}^{-1} \otimes \bar{\zeta}_{2},
\end{aligned}
$$

and again extended as an algebra map; in particular $\Delta_{\mathcal{A}\left(\underline{\widetilde{\mathbb{C}}_{\theta}^{4}}\right)}(\tilde{\rho})=\left(t_{1} t_{2}\right)^{-1} \otimes \tilde{\rho}$.

The $*$-involution on the generators $\zeta_{i}, \bar{\zeta}_{i}, i=1,2$ induced from the one in (2.18) reads

$$
\zeta_{1}^{\dagger}=q \bar{\zeta}_{1}, \quad \zeta_{2}^{\dagger}=-q^{-3} \bar{\zeta}_{2},
$$

from which it also follows that $\tilde{\rho}=q \zeta_{1} \zeta_{1}^{\dagger}+q^{3} \zeta_{2} \zeta_{2}^{\dagger}$. The corresponding noncommutative real variety is denoted $\widetilde{\mathbb{R}}_{\theta}^{4}$. Also on this second patch of the noncommutative four-sphere $S_{\theta}^{4}$, only for the coaction of $\mathcal{G}=\mathcal{A}\left(\left(\mathbb{R}^{\times}\right)^{2}\right)$ is there compatibility with the $*$-structure.

The intersection of the two open affine subvarieties $\mathbb{R}_{\theta}^{4}$ and $\widetilde{\mathbb{R}}_{\theta}^{4}$ is described by adjoining the element $\tilde{\rho}$ to $\mathcal{A}\left(\mathbb{R}_{\theta}^{4}\right)$ and $\rho$ to $\mathcal{A}\left(\widetilde{\mathbb{R}}_{\theta}^{4}\right)$. The corresponding gluing automorphism is a *-isomorphism $G: \mathcal{A}\left(\mathbb{R}_{\theta}^{4}\right)[\tilde{\rho}] \rightarrow[\rho] \mathcal{A}\left(\widetilde{\mathbb{R}}_{\theta}^{4}\right)$ in the category ${ }^{\mathcal{G}_{\theta}} \mathscr{\mathscr { M }}$ by [6, Prop. 3.25]. This morphism has a natural "geometric" interpretation. In the overlap of the two patches there are two sets of generators that describe "points", and $G$ describes how to pass from the affine coordinates $\xi_{j}$ to the affine coordinates $\zeta_{j}$; it is indeed just the identity map in terms of the homogeneous coordinates $\Lambda^{J}$ on the grassmannian, i.e. we do not "move" points, we simply describe how the coordinates of the two patches are related. Note that only the projection $\mathcal{A}\left(\mathbb{G r}_{\theta}(2 ; 4)\right) \rightarrow \mathcal{A}\left(\mathbb{R}_{\theta}^{4}\right)$ corresponding to the central generator $\Lambda^{(34)}$ is an algebra homomorphism, and it would be interesting to find a better interpretation of the two affine subvarieties as defining a "cover" in the noncommutative case $\theta \neq 0$.

2.5. Twistor bundle on $S_{\theta}^{4}$. The noncommutative twistor algebra $\mathcal{A}^{\text {tw }}=\mathcal{A}\left(\mathbb{C P}_{\theta}^{3}\right)$ is a particular instance of the noncommutative projective spaces described in $\S 1.5$; it is the homogeneous coordinate algebra generated by $w_{i}, i=1,2,3,4$ and the relations

$$
\begin{aligned}
& w_{i} w_{k}=w_{k} w_{i}, \quad i=1,2,3,4, k=3,4, \\
& w_{1} w_{2}=q^{2} w_{2} w_{1} .
\end{aligned}
$$


It is dual to the (complex) twistor space of the noncommutative sphere $S_{\theta}^{4}$. With $q \in \mathbb{R}$, there is a natural real structure on $\mathcal{A}^{\text {tw }}$ such that

$$
w_{1}^{\dagger}=w_{2}, \quad w_{2}^{\dagger}=w_{1}, \quad w_{3}^{\dagger}=w_{4}, \quad w_{4}^{\dagger}=w_{3} .
$$

At the level of the noncommutative correspondence algebra $\mathcal{A}\left(\mathbb{F} l_{\theta}(1,2 ; 4)\right)$, regarded as an $\mathcal{A}\left(\mathbb{G r}_{\theta}(2 ; 4)\right)$-bimodule, the Plücker equations (2.7) in the localized coordinate algebra $\mathcal{A}\left(\mathbb{F} l_{\theta}(1,2 ; 4)\right)\left[\Lambda^{(34)-1}\right]_{0}$ now read

$$
w_{1}=-w_{3} \xi_{1}-w_{4} \bar{\xi}_{2}, \quad w_{2}=-w_{3} \xi_{2}-w_{4} \bar{\xi}_{1} .
$$

Using the commutation relations (2.6) together with the multiplication rules of noncommutative Ore localization, one easily checks that the generators $w_{3}, w_{4}$ commute not only among themselves but also with $\xi_{i}, \bar{\xi}_{i}, i=1,2$, and it follows that

$$
\mathcal{A}\left(\mathbb{F} l_{\theta}(1,2 ; 4)\right)\left[\Lambda^{(34)-1}\right]_{0} \cong \mathcal{A}\left(\mathbb{R}_{\theta}^{4}\right) \otimes \mathcal{A}\left(\mathbb{C P}^{1}\right),
$$

with $\mathcal{A}\left(\mathbb{C P}^{1}\right)=\mathbb{C}\left[w_{3}, w_{4}\right]$ the homogeneous coordinate algebra of a commutative projective line $\mathbb{C P}^{1}$. This isomorphism implies that, in the image of the restriction functor $j^{\bullet}: \operatorname{coh}\left(\mathbb{G r}_{\theta}(2 ; 4)\right) \rightarrow \operatorname{coh}\left(\mathbb{R}_{\theta}^{4}\right)$ induced by localization on the real grassmannian, the tautological bundle $\mathcal{S}_{\theta}$ obtained through the twistor transform restricts to the free right $\mathcal{A}\left(\mathbb{R}_{\theta}^{4}\right)$-module of rank two, spanned by $w_{3}$ and $w_{4}$.

The situation is somewhat different for the noncommutative localization with respect to the minor $\Lambda^{(12)}$. The Plücker equations $(2.7)$ in ${ }_{0}\left[\Lambda^{(12)-1}\right] \mathcal{A}\left(\mathbb{F} l_{\theta}(1,2 ; 4)\right)$ are

$$
w_{3}=-q \bar{\zeta}_{1} w_{1}+q^{-1} \bar{\zeta}_{2} w_{2}, \quad w_{4}=q \zeta_{2} w_{1}-q^{-1} \zeta_{1} w_{2} .
$$

Now the generators $w_{1}, w_{2}$ do not commute with $\zeta_{i}, \bar{\zeta}_{i}, i=1,2$ in general: one finds that the localized coordinate algebra has the structure of the braided tensor product algebra

$$
{ }_{0}\left[\Lambda^{(12)-1}\right] \mathcal{A}\left(\mathbb{F} l_{\theta}(1,2 ; 4)\right) \cong \mathcal{A}\left(\mathbb{C P}_{\theta}^{1}\right) \otimes_{\theta} \mathcal{A}\left(\widetilde{\mathbb{R}}_{\theta}^{4}\right)
$$

where the noncommutative projective line $\mathbb{C P}_{\theta}^{1}$ has homogeneous coordinates $w_{1}, w_{2}$ subject to the relations in $(2.25)$. Coherent sheaves on $\mathbb{C P}_{\theta}^{1}$ can be functorially identified with coherent sheaves on a commutative line $\mathbb{C P}^{1}[6$, Prop. 4.2$]$, and hence, in the image of the restriction functor $\tilde{j} \bullet: \operatorname{coh}\left(\mathbb{G r}_{\theta}(2 ; 4)\right) \rightarrow \operatorname{coh}\left(\widetilde{\mathbb{R}}_{\theta}^{4}\right)$ induced by the localization, the tautological bundle $\mathcal{S}_{\theta}$ restricts to the free right $\mathcal{A}\left(\widetilde{\mathbb{R}}_{\theta}^{4}\right)$-module of rank two.

The free modules $\mathcal{A}\left(\mathbb{R}_{\theta}^{4}\right)^{\oplus 2}$ and $\mathcal{A}\left(\widetilde{\mathbb{R}}_{\theta}^{4}\right)^{\oplus 2}$ carry natural $*$-involutions induced by $(2.22)$, (2.24) and (2.26), and there is naturally a $*$-isomorphism $G_{2}: \mathcal{A}\left(\mathbb{R}_{\theta}^{4}\right)[\tilde{\rho}]^{\oplus 2} \rightarrow[\rho] \mathcal{A}\left(\widetilde{\mathbb{R}}_{\theta}^{4}\right)^{\oplus 2}$ in the category ${ }^{{ }^{9}} \mathscr{M}$ which is compatible with the module structures. This describes the twistor fibration over the noncommutative sphere $S_{\theta}^{4}$.

2.6. Braided ADHM parametrization of instanton moduli. We shall now study framed sheaves on Open $\left(\mathbb{C P}_{\theta}^{2}\right)$ and relate their moduli to a noncommutative deformation of the standard ADHM data.

Instanton moduli spaces. With $q=\exp \left(\frac{\mathrm{i}}{2} \theta\right)$ and $\theta \in \mathbb{C}$, consider the homogeneous coordinate algebra $\mathcal{A}=\mathcal{A}\left(\mathbb{C P}_{\theta}^{2}\right)$ whose generators have relations

$$
w_{1} w_{2}=q^{2} w_{2} w_{1}, \quad w_{1} w_{3}=w_{3} w_{1}, \quad w_{2} w_{3}=w_{3} w_{2} .
$$

Let $\mathcal{A}_{\infty}:=\mathcal{A} /\left\langle w_{3}\right\rangle$; we identify $\mathcal{A}_{\infty}=\mathcal{A}\left(\mathbb{C P}_{\theta}^{1}\right)$ as the homogeneous coordinate algebra dual to a noncommutative projective line $\mathbb{C P}_{\theta}^{1}$. The algebra projection $p: \mathcal{A} \rightarrow \mathcal{A}_{\infty}$ is dual to a closed embedding $\mathbb{C P}_{\theta}^{1} \hookrightarrow \mathbb{C P}_{\theta}^{2}$ of noncommutative projective varieties. Let 
$i: \mathcal{A}_{\infty} \hookrightarrow \mathcal{A}$ be the algebra inclusion. Then the inclusion functor $i \bullet$ gives a map from $\mathcal{A}$-modules to $\mathcal{A}_{\infty}$-modules, $M \mapsto M_{\infty}:=M /\left(M \cdot\left\langle w_{3}\right\rangle\right)$. There is a natural equivalence of abelian categories $\operatorname{coh}\left(\mathbb{C P}_{\theta}^{1}\right) \cong \operatorname{coh}\left(\mathbb{C P}^{1}\right)$ for any $\theta \in \mathbb{C}[6$, Prop. 4.2], which enables us to exploit the known cohomology of sheaves on the commutative projective line $\mathbb{C P}^{1}$.

Let us fix complex vector spaces $V$ and $W$ of dimensions $k$ and $r$, respectively. A framed sheaf on $\mathbb{C P}_{\theta}^{2}$ is a coherent torsion free sheaf $E$ on Open $\left(\mathbb{C P}_{\theta}^{2}\right)$ together with isomorphisms $H^{1}\left(\mathbb{C P}_{\theta}^{2}, E(-1)\right) \cong V$ and $\varphi: E_{\infty}:=i^{\bullet}(E) \rightarrow W \otimes \mathcal{O}_{\mathbb{C P}_{\theta}^{1}}$; the map $\varphi$ is called a framing of $E$ at infinity. A morphism of framed sheaves $(E, \varphi)$ and $\left(E^{\prime}, \varphi^{\prime}\right)$ is a homomorphism of $\mathcal{A}$-modules $\xi: E \rightarrow E^{\prime}$ which preserves the framing isomorphisms, i.e. $\varphi^{\prime} \circ i^{\bullet}(\xi)=\varphi$. The instanton moduli space $\mathcal{M}_{\theta}(r, k)$ is the set of isomorphism classes $[(E, \varphi)]$ of framed sheaves $(E, \varphi)$. In $[6$, Cor. 4.8$]$ it is shown that an isomorphism class $[(E, \varphi)] \in \mathcal{M}_{\theta}(r, k)$ has invariants

$$
\operatorname{rank}(E)=r, \quad \chi(E)=r-k,
$$

where $\chi(E):=\sum_{p \geq 0}(-1)^{p} \operatorname{dim}_{\mathbb{C}} H^{p}\left(\mathbb{C P}_{\theta}^{2}, E\right)$ is the noncommutative Euler characteristic of the framed sheaf $E$.

Our goal now is to reduce the study of moduli of framed sheaves on Open $\left(\mathbb{C P}_{\theta}^{2}\right)$ to a problem of linear algebra, defining a noncommutative deformation of the standard ADHM data. For this, we consider quadruples

$$
(2.31)\left(B_{1}, B_{2}, I, J\right) \in \mathcal{X}(W, V):=\operatorname{End}_{\mathbb{C}}(V)^{\oplus 2} \oplus \operatorname{Hom}_{\mathbb{C}}(W, V) \oplus \operatorname{Hom}_{\mathbb{C}}(V, W) .
$$

When we consider $\mathcal{H}_{\theta}$-coequivariant sheaves, there are natural coactions of the cotwisted Hopf algebra $\mathcal{H}_{\theta}$ induced by the torus $T=\left(\mathbb{C}^{\times}\right)^{2}$ on the moduli spaces. The finitedimensional vector spaces $V$ and $W$ are then $\mathcal{H}_{\theta}$-comodules, i.e. objects of the tensor category ${ }^{\mathcal{H}_{\theta}} \mathscr{M}$, and so are the vector spaces $\operatorname{End}_{\mathbb{C}}(V) \cong V^{*} \otimes V$ and $\operatorname{Hom}_{\mathbb{C}}(W, V) \cong$ $W^{*} \otimes V$. The explicit coaction on the quadruples (2.31) is given by $[6, \S 4.2]$

$$
\Delta_{X(W, V)}\left(B_{1}, B_{2}, I, J\right)=\left(t_{1} \otimes B_{1}, t_{2} \otimes B_{2},\left(t_{1} t_{2}\right) \otimes I, 1 \otimes J\right) .
$$

Here we only regard the parameter subspace $\operatorname{End}_{\mathbb{C}}(V)$ as a vector space object of $\mathcal{H}_{\theta} \mathscr{M}$, hence we do not deform the product on the endomorphism algebra where the matrices $B_{i}$ live. The variety $\mathcal{M}_{\theta}^{\mathrm{ADHM}}(r, k)$ of noncommutative complex ADHM data is the locally closed subvariety of quadruples (2.31) subject to the following two conditions:

(I.1) Noncommutative complex ADHM equation:

$$
\left[B_{1}, B_{2}\right]_{-\theta}+I J=0
$$

in $\operatorname{End}_{\mathbb{C}}(V)$, where the braided commutator

$$
\left[B_{1}, B_{2}\right]_{\theta}:=B_{1} B_{2}-q^{2} B_{2} B_{1}
$$

is induced by the functorial isomorphism (1.3) of the category $\mathcal{H}_{\theta} \mathscr{M}$; indeed using (1.7) we have $\Psi^{\theta}\left(B_{1} \otimes B_{2}\right)=\mathcal{R}_{\theta}\left(t_{2} \otimes t_{1}\right) B_{2} \otimes B_{1}=q^{2} B_{2} \otimes B_{1}$.

(I.2) Stability: There are no proper $B_{i}$-invariant subspaces of $V$ for $i=1,2$ which contain the image of $I$.

The general linear group GL( $k$ ) of basis change automorphisms of the vector space $V$ acts naturally on the variety $\mathcal{M}_{\theta}^{\mathrm{ADHM}}(r, k)$ as

$$
g \triangleright\left(B_{1}, B_{2}, I, J\right)=\left(g B_{1} g^{-1}, g B_{2} g^{-1}, g I, J g^{-1}\right), \quad g \in \mathrm{GL}(k) .
$$


This action is free and proper [6, Lem. 4.20], and so the quasi-projective variety of closed $\mathrm{GL}(k)$-orbits on the space $\mathcal{M}_{\theta}^{\mathrm{ADHM}}(r, k)$ is given by the geometric invariant theory quotient

$$
\widehat{\mathcal{M}}_{\theta}^{\mathrm{ADHM}}(r, k):=\mathcal{M}_{\theta}^{\mathrm{ADHM}}(r, k) / \mathrm{GL}(k) .
$$

There is a natural (set theoretic) bijection between the moduli space $\widehat{\mathcal{M}}_{\theta}^{\mathrm{ADHM}}(r, k)$ of braided linear algebraic ADHM data and the moduli space $\mathcal{M}_{\theta}(r, k)$ of framed sheaves on Open $\left(\mathbb{C P}_{\theta}^{2}\right)$. The proof of this result is quite technical, see $[6, \S 4.3]$; here we only briefly highlight the two essential steps that we need in the following:

1. Realise framed sheaves on Open $\left(\mathbb{C P}_{\theta}^{2}\right)$ as the middle cohomology of a linear monad complex

$$
\text { ․․ }: 0 \longrightarrow V \otimes \mathcal{O}_{\mathbb{C P}_{\theta}^{2}}(-1) \stackrel{\sigma}{\longrightarrow} V_{0} \otimes \mathcal{O}_{\mathbb{C P}_{\theta}^{2}} \stackrel{\tau}{\longrightarrow} V \otimes \mathcal{O}_{\mathbb{C P}_{\theta}^{2}}(1) \longrightarrow 0
$$

with $V_{0}=V \oplus V \oplus W$. Conversely, essentially any linear monad $\underline{\mathcal{e}} \bullet$ on $\mathbb{C P}_{\theta}^{2}$ of this form defines an isomorphism class in $\mathcal{M}_{\theta}(r, k)$, see [6, Thm. 4.25].

2. Show that the ADHM moduli space (2.35) has a similar monadic description. Given a quadruple of ADHM data $\left(B_{1}, B_{2}, I, J\right) \in \mathcal{M}_{\theta}^{\mathrm{ADHM}}(r, k)$, we define canonical sheaf morphisms

$$
\sigma=\left(\begin{array}{c}
B_{1} w_{3}+q w_{1} \\
B_{2} w_{3}+q^{-1} w_{2} \\
J w_{3}
\end{array}\right): V \otimes \mathcal{O}_{\mathbb{C P}_{\theta}^{2}}(-1) \longrightarrow V_{0} \otimes \mathcal{O}_{\mathbb{C P}_{\theta}^{2}}
$$

and

$$
\tau=\left(B_{1} w_{3}+q^{-1} w_{1} \quad B_{2} w_{3}+q w_{2} \quad I w_{3}\right): V_{0} \otimes \mathcal{O}_{\mathbb{C P}_{\theta}^{2}} \longrightarrow V \otimes \mathcal{O}_{\mathbb{C P}_{\theta}^{2}}(1)
$$

These maps determine a chain $\underline{\mathcal{C}}$ • of morphisms of coherent sheaves on Open $\left(\mathbb{C P}_{\theta}^{2}\right)$, which by [6, Thm. 4.32] is a monad of the type considered above. Every framed torsion free sheaf on $\mathbb{C P}_{\theta}^{2}$ arises from this construction.

In the next section we will describe this correspondence as an isomorphism between smooth algebraic varieties.

Instanton charge one. In the case $k=1$ we have $B_{i}=b_{i} \in \mathbb{C}$ for $i=1,2$, and we can regard the morphisms $I$ and $J$ as vectors

$$
I=\left(i_{1}, \ldots, i_{r}\right) \in W^{*}, \quad J=\left(\begin{array}{c}
j_{1} \\
\vdots \\
j_{r}
\end{array}\right) \in W .
$$

The braided ADHM equation (2.33) then defines a quadric in $\mathbb{C}^{2 r+2}$ given by

$$
\left(1-q^{-2}\right) b_{1} b_{2}+\sum_{l=1}^{r} i_{l} j_{l}=0 \text {. }
$$

Stability is equivalent to $i_{l} \neq 0$ for at least one index $l=1, \ldots, r$, showing that the moduli space $\widehat{\mathcal{M}}_{\theta}^{\mathrm{ADHM}}(r, 1)$ is quasi-projective. An element $t \in \mathrm{GL}(1)=\mathbb{C}^{\times}$acts trivially on $b_{1}, b_{2}$, and as multiplication by $t$ on $i_{l}$ and by $t^{-1}$ on $j_{l}$ for each $l=1, \ldots, r$. In the patch $i_{r} \neq 0$ we can use this scaling symmetry to set $i_{r}=1$, and then use (2.38) to eliminate $j_{r} \in \mathbb{C}$. This coordinatizes $\widehat{\mathcal{M}}_{\theta}^{\mathrm{ADHM}}(r, 1)$ as a patch $\mathbb{C}^{2} \times \mathbb{C}^{r-1} \times \mathbb{C}^{r-1}$. The 
last factor is the cotangent space at the point $[I] \in \mathbb{P}\left(W^{*}\right) \cong \mathbb{C P}^{r-1}$ : with $i_{r}=1$ and $j_{r}=-\left(1-q^{-2}\right) b_{1} b_{2}$, the quadric (2.38) reads

$$
\sum_{l=1}^{r-1} i_{l} j_{l}=0
$$

which is an equation for $\left(j_{1}, \ldots, j_{r-1}\right) \in T_{[I]}^{*} \mathbb{C P}^{r-1}$. This gives the charge 1 instanton moduli space

$$
\mathcal{M}_{\theta}(r, 1) \cong \mathbb{C}^{2} \times T^{*} \mathbb{C P}^{r-1}
$$

as a complex variety for all $\theta \in \mathbb{C}$ and $r \geq 1$.

Self-conjugate instanton modules. We will now provide another characterization of the moduli space $\mathcal{M}_{\theta}(r, k)$ in terms of "real" linear algebraic data. For this, we fix hermitean inner products on the complex vector spaces $V$ and $W$. Then, with $q \in \mathbb{R}$, the space of quadruples $\mathcal{X}(W, V)$ in (2.31) acquires a natural quaternionic structure given by the conjugate linear anti-involution

$$
\mathscr{J}: X(W, V) \longrightarrow X(W, V), \quad \mathscr{J}\left(B_{1}, B_{2}, I, J\right)=\left(-B_{2}^{\dagger}, B_{1}^{\dagger},-J^{\dagger}, I^{\dagger}\right)
$$

extended as an anti-algebra map. The variety $\mathcal{M}_{\theta}^{\mathbb{R}}(r, k)$ of noncommutative real ADHM data is the subspace of $\mathcal{M}_{\theta}^{\mathrm{ADHM}}(r, k)$ consisting of quadruples (2.31) which satisfy, in addition to conditions (I.1) and (I.2) of the complex data, the real ADHM equation

$$
\left[B_{1}, B_{1}^{\dagger}\right]_{-\theta}+q^{-2}\left[B_{2}, B_{2}^{\dagger}\right]_{\theta}+I I^{\dagger}-J^{\dagger} J=0
$$

in $\operatorname{End}_{\mathbb{C}}(V)$. The natural $\mathrm{GL}(k)$-action on $\mathcal{M}_{\theta}^{\mathrm{ADHM}}(r, k)$ reduces on $\mathcal{M}_{\theta}^{\mathbb{R}}(r, k)$ to an action of the group $\mathrm{U}(k)$ of unitary basis change automorphisms of the vector space $V$. The corresponding space of stable orbits is denoted $\widehat{\mathcal{M}}_{\theta}^{\mathrm{tw}}(r, k)$. This quotient is related to a particular class of framed torsion free sheaves on $\mathbb{C P}_{\theta}^{2}$, as we explain momentarily.

Let us first make two remarks concerning the map defined by (2.41):

- We can rewrite the real ADHM equation (2.42) as

$$
\left[\mathscr{J}\left(B_{1}\right), B_{2}\right]_{-\theta}+\left[B_{1}, \mathscr{J}\left(B_{2}\right)\right]_{-\theta}+\mathscr{J}(I) J+I \mathscr{J}(J)=0 \text {. }
$$

This is precisely the equation that would arise if we let the quaternionic structure $\mathscr{J}$ act on the complex ADHM equation (2.33) as a derivation, rather than as an anti-algebra map; hence if we interpret $\mathscr{J}$ as a symmetry generator in this way then the real ADHM equation is automatically satisfied.

- Both the complex ADHM equation (2.33) and the real ADHM equation (2.42) are invariant for the full coaction associated to $T$. On the other hand, requiring that the quaternionic structure in $(2.41)$ be coequivariant, i.e. $(\mathrm{id} \otimes \mathscr{J}) \circ \Delta_{X(W, V)}=$ $\Delta_{X(W, V)} \circ \mathscr{J}$ so that it is a morphism in the category ${ }^{\mathcal{H}_{\theta}} \mathscr{M}$ of left $\mathcal{H}_{\theta}$-comodules, restricts the toric symmetry to a coaction associated with the real subgroup $G \subset T$ with $\left(t_{1}\right)^{*}=t_{2}$. Here we take $B_{i}^{\dagger}$ to transform with weight $t_{i}^{*}$ for $i=1,2, J^{\dagger}$ with weight $\left(t_{1} t_{2}\right)^{*}$, and $I^{\dagger}$ to be coinvariant.

We will now realize a correspondence between $\widehat{\mathcal{M}}_{\theta}^{\mathbb{R}}(r, k)$ and linear monads on $\mathbb{C P}_{\theta}^{3}$. We endow the noncommutative twistor algebra $\mathcal{A}^{\text {tw }}=\mathcal{A}\left(\mathbb{C P}_{\theta}^{3}\right)$ with a conjugate linear anti-involution $\mathscr{J}: \mathcal{A}^{\mathrm{tw}} \rightarrow\left(\mathcal{A}^{\mathrm{tw}}\right)^{\text {op }}$ acting on generators as the quaternionic structure

$$
\mathscr{J}\left(w_{1}, w_{2}, w_{3}, w_{4}\right)=\left(w_{2},-w_{1}, w_{4},-w_{3}\right) \text {. }
$$


There is a natural embedding of the noncommutative projective plane $\mathbb{C P}_{\theta}^{2}$ into the noncommutative twistor space $\mathbb{C P}_{\theta}^{3}$. The homogeneous coordinate algebra of the original $\mathbb{C P}_{\theta}^{2}$ is recovered through $\mathcal{A} \cong \mathcal{A}^{\mathrm{tw}} /\left\langle w_{4}\right\rangle$. Let $\iota: \mathcal{A} \hookrightarrow \mathcal{A}^{\mathrm{tw}}$ be the natural algebra inclusion. We again denote by $i: \mathcal{A}_{\infty} \hookrightarrow \mathcal{A}^{\text {tw }}$ the algebra inclusion of the noncommutative projective line $\mathbb{C P}_{\theta}^{1}$ with $\mathcal{A}_{\infty} \cong \mathcal{A}^{\mathrm{tw}} /\left\langle w_{3}, w_{4}\right\rangle$. Consider a linear monad on $\mathbb{C P}_{\theta}^{3}$ of the form

$$
\underline{\mathrm{e}}^{\mathrm{tw}}: 0 \longrightarrow V \otimes \mathcal{O}_{\mathbb{C P}_{\theta}^{3}}(-1) \stackrel{\sigma}{\longrightarrow} V_{0} \otimes \mathcal{O}_{\mathbb{C P}_{\theta}^{3}} \stackrel{\tau}{\longrightarrow} V \otimes \mathcal{O}_{\mathbb{C P}_{\theta}^{3}}(1) \longrightarrow 0 \text {. }
$$

Its restriction $i^{\bullet}\left(\underline{\mathcal{e}}^{\text {tw }}\right)$ is again a monad on $\mathbb{C P}_{\theta}^{1}$ which is quasi-isomorphic to $W \otimes \mathcal{O}_{\mathbb{C P}_{\theta}^{1}}$. The anti-homomorphism $\mathscr{J}$ induces a functor $\mathscr{J} \bullet$ between categories of sheaves which, when extended to the derived category of $\operatorname{coh}\left(\mathbb{C P}_{\theta}^{3}\right)$ and applied to a monad $\underline{\mathfrak{e}} \bullet$,w , gives a monad

$$
\underline{\mathcal{e}}^{\mathrm{tw}}{ }^{\dagger}: 0 \longrightarrow \bar{V}^{*} \otimes \mathcal{O}_{\mathbb{C P}_{\theta}^{3}}(-1) \stackrel{\sigma^{\dagger}}{\longrightarrow} \bar{V}_{0}^{*} \otimes \mathcal{O}_{\mathbb{C P}_{\theta}^{3}} \stackrel{\tau^{\dagger}}{\longrightarrow} \bar{V}^{*} \otimes \mathcal{O}_{\mathbb{C P}_{\theta}^{3}}(1) \longrightarrow 0 .
$$

Here the bars denote complex conjugation while $\sigma^{\dagger}:=\mathscr{J}^{\bullet}(\sigma)^{*}$ and $\tau^{\dagger}:=\mathscr{J}^{\bullet}(\tau)^{*}$. We say that a monad $\underline{\mathcal{e}} \underline{\bullet}^{\text {tw }}$ is self-conjugate if there is an isomorphism $\underline{\mathcal{e}} \mathscr{\bullet}^{\dagger} \cong \underline{\mathcal{e}} \bullet$ tw of complexes.

Then there is a natural (set-theoretic) bijective correspondence between isomorphism classes of self-conjugate linear monad complexes $\underline{\mathcal{e}} \underline{\bullet}^{\mathrm{tw}}$ on $\mathbb{C P}_{\theta}^{3}$ and isomorphism classes in the moduli space $\widehat{\mathcal{M}}_{\theta}^{\mathbb{R}}(r, k)$ of braided real ADHM data; the proof follows a similar argument to that sketched above, once we take into account the real structure (see [6, Thm. 4.40] for details). The restriction $\iota^{\bullet}\left(\underline{\mathcal{e}}^{\mathbb{R}}\right)$ of a self-conjugate monad on $\mathbb{C P}_{\theta}^{3}$ is a monad on $\mathbb{C P}_{\theta}^{2}$ which we know defines an isomorphism class in $\mathcal{M}_{\theta}(r, k)$. This gives a map of moduli spaces $\widehat{\mathcal{M}}_{\theta}^{\mathbb{R}}(r, k) \rightarrow \mathcal{M}_{\theta}(r, k)$. At the level of noncommutative ADHM data, this map is just the natural inclusion of varieties $\mathcal{M}_{\theta}^{\mathbb{R}}(r, k) \hookrightarrow \mathcal{M}_{\theta}^{\mathrm{ADHM}}(r, k)$.

2.7. Construction of noncommutative instantons. We will now demonstrate that the smaller class of torsion free sheaves on Open $\left(\mathbb{C P}_{\theta}^{2}\right)$ parametrized by the moduli space $\widehat{\mathcal{M}}_{\theta}^{\mathbb{R}}(r, k)$ corresponds directly to a class of anti-selfdual connections on a canonically associated "instanton bundle" over the noncommutative sphere $S_{\theta}^{4}$.

Instanton modules. We use the monadic description of noncommutative real ADHM data on the twistor space $\mathbb{C P}_{\theta}^{3}$ to construct canonical bundles, called "instanton bundles", on the noncommutative sphere $S_{\theta}^{4}$. This is achieved via the twistor transform of real framed torsion free sheaves with $E^{\dagger} \cong E$ on Open $\left(\mathbb{C P}_{\theta}^{3}\right)$ via the noncommutative correspondence diagram (2.8). We can apply the derived functor of the twistor transform $p_{2}{ }^{*} p_{1 *}$ to a self-conjugate linear monad complex $\underline{\mathfrak{e}}_{\bullet}^{\text {tw }}$ on $\mathbb{C P}_{\theta}^{3}$ to get the complex

$$
p_{2}^{*} p_{1 *}\left(\underline{\mathfrak{e}}^{\mathrm{tw}}\right): 0 \longrightarrow V_{0} \otimes \mathcal{O}_{\mathbb{G r}_{\theta}(2 ; 4)} \stackrel{\left(\mathrm{id}_{V} \otimes \hat{\eta}\right) \circ \tau}{\longrightarrow} V \otimes \mathcal{S}_{\theta} \longrightarrow 0
$$

where $\hat{\eta}$ is the rank two projector which defines the tautological bundle over the Grassmann variety as $\mathcal{S}_{\theta} \cong \hat{\eta}\left(\mathcal{A}\left(\mathbb{G r}_{\theta}(2 ; 4)\right)^{\oplus 4}\right)$ (via the noncommutative Euler sequence, see [6, eq. (3.7)]). It follows that the image of a cohomology sheaf $E=H^{0}\left(\underline{\mathfrak{e}}^{\mathrm{tw}}\right)$ under the twistor transform is the sheaf on Open $\left(\mathbb{G r}_{\theta}(2 ; 4)\right)$ given by

$$
E^{\prime}=p_{2}{ }^{*} p_{1 *}(E)=\operatorname{ker}((\mathrm{id} \otimes \hat{\eta}) \circ \tau) .
$$

It follows from (2.28) that the restriction of the complex (2.44) to the patch $\mathbb{R}_{\theta}^{4}$ of the noncommutative sphere $S_{\theta}^{4}$ yields the "Dirac operator"

$$
\mathcal{D}:=j^{\bullet}(\mathrm{id} \otimes \hat{\eta}) \circ \tau: V_{0} \otimes \mathcal{A}\left(\mathbb{R}_{\theta}^{4}\right) \longrightarrow(V \oplus V) \otimes \mathcal{A}\left(\mathbb{R}_{\theta}^{4}\right)
$$


which is written explicitly as

$$
\mathcal{D}=\left(\begin{array}{ccc}
B_{1}-q^{-1} \xi_{1} & B_{2}-q \xi_{2} & I \\
-B_{2}^{\dagger}-q^{-1} \bar{\xi}_{2} & B_{1}^{\dagger}-q \bar{\xi}_{1} & -J^{\dagger}
\end{array}\right) .
$$

The operator $\mathcal{D}$ is a surjective morphism of free right $\mathcal{A}\left(\mathbb{R}_{\theta}^{4}\right)$-modules and $\mathcal{D}^{\dagger}$ is injective; here the $\dagger$-involution is the tensor product of the real structure given by (2.22) and those of the chosen hermitean structures on the vector spaces $V$ and $W$. The "Laplace operator"

$$
\triangle:=\mathcal{D} \circ \mathcal{D}^{\dagger}:(V \oplus V) \otimes \mathcal{A}\left(\mathbb{R}_{\theta}^{4}\right) \longrightarrow(V \oplus V) \otimes \mathcal{A}\left(\mathbb{R}_{\theta}^{4}\right)
$$

is an isomorphism and there is a decomposition

$$
V_{0} \otimes \mathcal{A}\left(\mathbb{R}_{\theta}^{4}\right)=\operatorname{im}\left(\mathcal{D}^{\dagger}\right) \oplus \operatorname{ker}(\mathcal{D})
$$

of $\mathcal{A}\left(\mathbb{R}_{\theta}^{4}\right)$-modules [6, Prop. 5.5]. Moreover, the operators $\mathcal{D}, \mathcal{D}^{\dagger}$ and $\triangle$ are all $\mathcal{G}_{\theta^{-}}$ coequivariant morphisms. The right $\mathcal{A}\left(\mathbb{R}_{\theta}^{4}\right)$-module

$$
\mathcal{E}:=\operatorname{ker}(\mathcal{D})=\operatorname{ker}(j \bullet(\mathrm{id} \otimes \hat{\eta}) \circ \tau)
$$

is projective by (2.48). It is also finitely generated, and has $\operatorname{rank} \operatorname{dim}_{\mathbb{C}}(W)=r$ since $\mathcal{D}$ is surjective. The corresponding projection can be given as

$$
P:=\mathrm{id}-\mathcal{D}^{\dagger} \circ \triangle^{-1} \circ \mathcal{D}: V_{0} \otimes \mathcal{A}\left(\mathbb{R}_{\theta}^{4}\right) \longrightarrow \mathcal{E},
$$

with $P^{2}=P=P^{\dagger}$ and trace $\operatorname{Tr} P=\operatorname{dim}_{\mathbb{C}}\left(V_{0}\right)-\operatorname{dim}_{\mathbb{C}}(V \oplus V)=r$. The module (2.49) is called an instanton bundle over $\mathbb{R}_{\theta}^{4}$; it defines an object of the category ${ }^{G_{\theta}} \mathscr{M}$. One can show that the isomorphism class of $\mathcal{E}$ depends only on the class of the noncommutative ADHM data $\left(B_{1}, B_{2}, I, J\right)$ in the moduli variety $\mathcal{M}_{\theta}^{\mathbb{R}}(r, k)$, and that the reality condition $\mathcal{E}^{\dagger} \cong \mathcal{E}$ is satisfied. One can repeat these arguments to construct an instanton bundle $\widetilde{\mathcal{E}}$ on the second patch $\widetilde{\mathbb{R}}_{\theta}^{4}$ of $S_{\theta}^{4}$, and then use the gluing automorphism $G$ between the two patches to induce an isomorphism between the modules $\mathcal{E}[\tilde{\rho}]:=\mathcal{E} \otimes_{\mathcal{A}\left(\mathbb{R}_{\theta}^{4}\right)} \mathcal{A}\left(\mathbb{R}_{\theta}^{4}\right)[\tilde{\rho}]$ and $[\rho] \tilde{\mathcal{E}}:=[\rho] \mathcal{A}\left(\widetilde{\mathbb{R}}_{\theta}^{4}\right) \otimes_{\mathcal{A}\left(\widetilde{\mathbb{R}}_{\theta}^{4}\right)} \tilde{\mathcal{E}}$ which is compatible with the $\dagger$-involutions, thus defining a $\mathcal{S}_{\theta}$-coequivariant instanton bundle on the noncommutative sphere $S_{\theta}^{4}$.

Instanton gauge fields. We will now construct canonical connections on the instanton bundle. For this, let $\Omega_{\mathbb{R}^{4}}^{\bullet}=\bigwedge^{\bullet} \Omega_{\mathbb{R}^{4}}^{1}$ be the usual classical differential calculus on the coordinate algebra $\mathcal{A}\left(\mathbb{R}^{4}\right)$. It is generated as a differential graded algebra by degree zero elements $\xi_{i}, \bar{\xi}_{i}$ and degree one elements $\mathrm{d} \xi_{i}, \mathrm{~d} \bar{\xi}_{i}$ satisfying the skew-commutation relations

$$
\mathrm{d} \xi_{i} \wedge \mathrm{d} \xi_{j}=-\mathrm{d} \xi_{j} \wedge \mathrm{d} \xi_{i}, \quad \mathrm{~d} \xi_{i} \wedge \mathrm{d} \bar{\xi}_{j}=-\mathrm{d} \bar{\xi}_{j} \wedge \mathrm{d} \xi_{i}, \quad \mathrm{~d} \bar{\xi}_{i} \wedge \mathrm{d} \bar{\xi}_{j}=-\mathrm{d} \bar{\xi}_{j} \wedge \mathrm{d} \bar{\xi}_{i}
$$

and the symmetric $\mathcal{A}\left(\mathbb{R}^{4}\right)$-bimodule structure

$$
\xi_{i} \mathrm{~d} \xi_{j}=\mathrm{d} \xi_{j} \xi_{i}, \quad \xi_{i} \mathrm{~d} \bar{\xi}_{j}=\mathrm{d} \bar{\xi}_{j} \xi_{i}, \quad \bar{\xi}_{i} \mathrm{~d} \bar{\xi}_{j}=\mathrm{d} \bar{\xi}_{j} \bar{\xi}_{i}
$$

for $i, j=1,2$. The differential $\mathrm{d}: \Omega_{\mathbb{R}^{4}}^{0}:=\mathcal{A}\left(\mathbb{R}^{4}\right) \rightarrow \Omega_{\mathbb{R}^{4}}^{1}$ is defined by $\xi_{i} \mapsto \mathrm{d} \xi_{i}, \bar{\xi}_{i} \mapsto \mathrm{d} \bar{\xi}_{i}$ and extended uniquely to a map of degree one, $\mathrm{d}: \Omega_{\mathbb{R}^{4}}^{n} \rightarrow \Omega_{\mathbb{R}^{4}}^{n+1}$, with $\mathrm{d}^{2}=0$, using $\mathbb{C}$-linearity and the graded Leibniz rule

$$
\mathrm{d}\left(\omega \wedge \omega^{\prime}\right)=\mathrm{d} \omega \wedge \omega^{\prime}+(-1)^{\operatorname{deg}(\omega)} \omega \wedge \mathrm{d} \omega^{\prime}
$$

where the product is taken over $\mathcal{A}\left(\mathbb{R}^{4}\right)$ using the bimodule structure of $\Omega_{\mathbb{R}^{4}}^{\bullet}$. We will demand that the differential calculus on $\mathcal{A}\left(\mathbb{R}^{4}\right)$ is coequivariant for the real torus coaction $\Delta_{L}$ given by (2.21). Then we can extend this coaction to a left coaction $\Delta_{L}: \Omega_{\mathbb{R}^{4}}^{\bullet} \rightarrow \mathcal{G} \otimes \Omega_{\mathbb{R}^{4}}^{\bullet}$ such that $\Delta_{L}$ is an $\mathcal{A}\left(\mathbb{R}^{4}\right)$-bimodule morphism and $\mathrm{d}$ is a left $\mathcal{G}$-comodule morphism . We next use the Drinfel'd twist $F_{\theta}$ of $(1.6)$ to deform $\Omega_{\mathbb{R}^{4}}^{\bullet}$, following the general prescription 
for $\mathcal{G}$-comodule algebras. This defines the braided exterior product $\wedge_{\theta}$ as the projection of the tensor product on the quotient of the tensor algebra

$$
T_{\theta}\left(\Omega_{\mathbb{R}^{4}}^{1}\right)=\mathcal{A}\left(\mathbb{R}_{\theta}^{4}\right) \oplus \bigoplus_{n \geq 1}\left(\Omega_{\mathbb{R}^{4}}^{1}\right)^{\otimes_{\mathcal{A}\left(\mathbb{R}_{\theta}^{4}\right)}}
$$

by the ideal generated by the braided skew-commutation relations (see (1.12)). The undeformed differential $\mathrm{d}$ is still a derivation (of degree one) of the deformed product $\wedge_{\theta}$, as one can easily check. The above construction defines a canonical differential graded algebra $\Omega_{\mathbb{R}_{\theta}^{\bullet}}^{\bullet}=\bigwedge_{\theta}^{\bullet} \Omega_{\mathbb{R}^{4}}^{1}$ subject to the braided skew-commutation relations

$$
\begin{gathered}
\mathrm{d} \xi_{i} \wedge \mathrm{d} \xi_{j}=-q_{i j}^{2} \mathrm{~d} \xi_{j} \wedge \mathrm{d} \xi_{i} \quad, \quad \mathrm{~d} \bar{\xi}_{i} \wedge \mathrm{d} \bar{\xi}_{j}=-q_{i+1 j+1}^{2} \mathrm{~d} \bar{\xi}_{j} \wedge \mathrm{d} \bar{\xi}_{i} \\
\mathrm{~d} \xi_{i} \wedge \mathrm{d} \bar{\xi}_{j}=-q_{i j+1}^{2} \mathrm{~d} \bar{\xi}_{j} \wedge \mathrm{d} \xi_{i}
\end{gathered}
$$

and the braided symmetric $\mathcal{A}\left(\mathbb{R}_{\theta}^{4}\right)$-bimodule structure

$$
\begin{aligned}
& \xi_{i} \mathrm{~d} \xi_{j}=q_{i j}^{2} \mathrm{~d} \xi_{j} \xi_{i}, \quad \bar{\xi}_{i} \mathrm{~d} \bar{\xi}_{j}=q_{i+1 j+1}^{2} \mathrm{~d} \bar{\xi}_{j} \bar{\xi}_{i}, \\
& \xi_{i} \mathrm{~d} \bar{\xi}_{j}=q_{i j+1}^{2} \mathrm{~d} \bar{\xi}_{j} \xi_{i} \quad, \quad \bar{\xi}_{i} \mathrm{~d} \xi_{j}=q_{i+1 j}^{2} \mathrm{~d} \xi_{j} \bar{\xi}_{i} .
\end{aligned}
$$

Here we drop the explicit deformation symbols from the products. The real structure (2.22) extends to $\Omega_{\mathbb{R}_{\theta}^{\bullet}}^{\bullet}$ by graded extension of the morphism $\xi_{i} \mapsto \xi_{i}^{\dagger}$ and $\left(\mathrm{d} \xi_{i}\right)^{\dagger}=\mathrm{d}\left(\xi_{i}^{\dagger}\right)$.

We are ready to give a canonical connection on the right $\mathcal{A}\left(\mathbb{R}_{\theta}^{4}\right)$-module $\mathcal{E}$ : a linear map

$$
\nabla: \mathcal{E} \longrightarrow \mathcal{E} \otimes_{\mathcal{A}\left(\mathbb{R}_{\theta}^{4}\right)} \Omega_{\mathbb{R}_{\theta}^{4}}^{1}
$$

satisfying the Leibniz rule

$$
\nabla(\sigma \triangleleft f)=(\nabla \sigma) \triangleleft f+\sigma \otimes \mathrm{d} f
$$

for $f \in \mathcal{A}\left(\mathbb{R}_{\theta}^{4}\right)$ and $\sigma \in \mathcal{E}$. Let $\iota: \mathcal{E} \rightarrow V_{0} \otimes \mathcal{A}\left(\mathbb{R}_{\theta}^{4}\right)$ denote the natural inclusion, and $\mathrm{d}: \mathcal{A}\left(\mathbb{R}_{\theta}^{4}\right) \rightarrow \Omega_{\mathbb{R}_{\theta}^{4}}^{1}$ the differential introduced above. We then take $\nabla$ to be the Grassmann connection associated to the projection $P$, which is defined by the composition

$$
\nabla=P \circ \mathrm{d} \circ \iota
$$

It is easily seen to be compatible with the $\dagger$-involution, and its curvature is given by

$$
F_{\nabla}=\nabla^{2}=P(\mathrm{~d} P)^{2} \in \operatorname{Hom}_{\mathcal{A}\left(\mathbb{R}_{\theta}^{4}\right)}\left(\mathcal{E}, \mathcal{E} \otimes_{\mathcal{A}\left(\mathbb{R}_{\theta}^{4}\right)} \Omega_{\mathbb{R}_{\theta}^{4}}^{2}\right)
$$

Gauge transformations $g \in \operatorname{Aut}_{\mathcal{A}\left(\mathbb{R}_{\theta}^{4}\right)}(\mathcal{E})$ act naturally through $\nabla \mapsto g \nabla g^{-1}$, from which it follows that $F_{\nabla} \mapsto g F_{\nabla} g^{-1}$; the gauge equivalence class of the instanton connection $\nabla=$ $P \circ$ do $\iota$ depends only on the class of the noncommutative ADHM data $\left(B_{1}, B_{2}, I, J\right)$ in the moduli variety $\mathcal{M}_{\theta}^{\mathbb{R}}(r, k)[6$, Lem. 5.13]. Similarly one builds the instanton connection $\widetilde{\nabla}$ on the left $\mathcal{A}\left(\widetilde{\mathbb{R}}_{\theta}^{4}\right)$-module $\tilde{\mathcal{E}}$, with curvature $F_{\widetilde{\nabla}}=\widetilde{P}(\mathrm{~d} \widetilde{P})^{2} \in \operatorname{Hom}_{\mathcal{A}\left(\widetilde{\mathbb{R}}_{\theta}^{4}\right)}\left(\tilde{\mathcal{E}}, \Omega_{\widetilde{\mathbb{R}}_{\theta}^{4}}^{2} \otimes_{\mathcal{A}\left(\widetilde{\mathbb{R}}_{\theta}^{4}\right)} \tilde{\mathcal{E}}\right)$.

The instanton connections $\nabla_{\tilde{\rho}}$ and ${ }_{\rho} \widetilde{\nabla}$ on the adjoinment bundles $\mathcal{\varepsilon}[\tilde{\rho}]$ and $[\rho] \tilde{\mathcal{E}}$, respectively, are related by a gauge transformation, which thereby defines the instanton connection on the noncommutative sphere $S_{\theta}^{4}$. 
BPS equations. We will now demonstrate that the Grassmann connections constructed above define noncommutative instantons in analogy with the classical case, i.e. that they satisfy some form of anti-selfduality equations with respect to a suitable Hodge duality operator acting on two-forms. The classical Hodge duality operator on two-forms $*: \Omega_{\mathbb{R}^{4}}^{2} \rightarrow \Omega_{\mathbb{R}^{4}}^{2}$, induced by the euclidean metric on $\mathbb{R}^{4}$, is given by

$$
\begin{array}{lll}
*\left(\mathrm{~d} \xi_{1} \wedge \mathrm{d} \bar{\xi}_{2}\right)=-\mathrm{d} \xi_{1} \wedge \mathrm{d} \bar{\xi}_{2} & *\left(\mathrm{~d} \xi_{1} \wedge \mathrm{d} \xi_{2}\right)=\mathrm{d} \xi_{1} \wedge \mathrm{d} \xi_{2}, \\
*\left(\mathrm{~d} \xi_{1} \wedge \mathrm{d} \bar{\xi}_{1}\right)=-\mathrm{d} \xi_{2} \wedge \mathrm{d} \bar{\xi}_{2} & , & *\left(\mathrm{~d} \xi_{2} \wedge \mathrm{d} \bar{\xi}_{2}\right)=-\mathrm{d} \xi_{1} \wedge \mathrm{d} \bar{\xi}_{1} \\
*\left(\mathrm{~d} \bar{\xi}_{1} \wedge \mathrm{d} \bar{\xi}_{2}\right)=\mathrm{d} \bar{\xi}_{1} \wedge \mathrm{d} \bar{\xi}_{2} & *\left(\mathrm{~d} \xi_{2} \wedge \mathrm{d} \bar{\xi}_{1}\right)=-\mathrm{d} \xi_{2} \wedge \mathrm{d} \bar{\xi}_{1} .
\end{array}
$$

In contrast to the case of isospectral deformations [3], the coaction (2.21) of the real torus algebra $\mathcal{G}=\mathcal{A}\left(\left(\mathbb{R}^{\times}\right)^{2}\right)$ on $\mathcal{A}\left(\mathbb{R}^{4}\right)$ is not isometric. However, it does coact by conformal transformations on $\mathcal{A}\left(\mathbb{R}^{4}\right)$, and hence preserves the Hodge operator. This is easily checked using (2.52) and (2.21) which shows that the operator $*: \Omega_{\mathbb{R}^{4}}^{2} \rightarrow \Omega_{\mathbb{R}^{4}}^{2}$ is coequivariant,

$$
\Delta_{L}(* \omega)=(\mathrm{id} \otimes *) \Delta_{L}(\omega) \quad \text { for } \quad \omega \in \Omega_{\mathbb{R}^{4}}^{2},
$$

and hence defines a morphism of the category ${ }^{9} \mathscr{M}$. Since the vector space $\Omega_{\mathbb{R}_{\theta}^{4}}^{2}$ coincides with its classical counterpart $\Omega_{\mathbb{R}^{4}}^{2}$, and the quantization functor $\mathscr{F}_{\theta}:{ }^{\mathcal{H}} \mathscr{M} \rightarrow{ }^{\mathcal{H}_{\theta}} \mathscr{M}$ acts as the identity on objects and morphisms of ${ }^{\mathcal{H}} \mathscr{M}$, we can define a Hodge duality operator $*_{\theta}: \Omega_{\mathbb{R}_{\theta}^{4}}^{2} \rightarrow \Omega_{\mathbb{R}_{\theta}^{4}}^{2}$ by the same formula $(2.52)$, which by construction is a morphism in the category ${ }^{{ }_{\theta}} \mathscr{M}$. In particular, it satisfies $*_{\theta}^{2}=$ id and the $\mathcal{G}_{\theta}$-coequivariant decomposition

$$
\Omega_{\mathbb{R}_{\theta}^{4}}^{2}=\Omega_{\mathbb{R}_{\theta}^{4}}^{2,+} \oplus \Omega_{\mathbb{R}_{\theta}^{4}}^{2,-},
$$

of the right $\mathcal{A}\left(\mathbb{R}_{\theta}^{4}\right)$-module $\Omega_{\mathbb{R}_{\theta}^{4}}^{2}$ into submodules corresponding to eigenvalues \pm 1 of $*_{\theta}$, is identical as a vector space to that of the classical case. Hence the eigenmodules of selfdual and anti-selfdual two-forms are given respectively by

$$
\begin{aligned}
& \Omega_{\mathbb{R}_{\theta}^{4}}^{2,+}=\mathcal{A}\left(\mathbb{R}_{\theta}^{4}\right)\left\langle\mathrm{d} \xi_{1} \wedge \mathrm{d} \xi_{2}, \mathrm{~d} \bar{\xi}_{1} \wedge \mathrm{d} \bar{\xi}_{2}, \mathrm{~d} \xi_{1} \wedge \mathrm{d} \bar{\xi}_{1}-\mathrm{d} \xi_{2} \wedge \mathrm{d} \bar{\xi}_{2}\right\rangle \\
& \Omega_{\mathbb{R}_{\theta}^{4}}^{2,-}=\mathcal{A}\left(\mathbb{R}_{\theta}^{4}\right)\left\langle\mathrm{d} \xi_{1} \wedge \mathrm{d} \bar{\xi}_{2}, \mathrm{~d} \xi_{2} \wedge \mathrm{d} \bar{\xi}_{1}, \mathrm{~d} \xi_{1} \wedge \mathrm{d} \bar{\xi}_{1}+\mathrm{d} \xi_{2} \wedge \mathrm{d} \bar{\xi}_{2}\right\rangle .
\end{aligned}
$$

Then the curvature $F_{\nabla}$ of the instanton connection is anti-selfdual, i.e. as a two-form it obeys the anti-selfduality equation (with the notational simplification id $\otimes *_{\theta} \simeq *_{\theta}$ )

$$
*_{\theta} F_{\nabla}=-F_{\nabla} .
$$

The proof can be found in [6, Prop. 5.21], and it consists in showing that $F_{\nabla}$ is proportional to $\mathrm{d}^{\dagger} \wedge \mathrm{d} \mathcal{D}$ as a two-form. Once the exterior derivative of the Dirac operator (2.46) and its adjoint are computed, using the commutation relations (2.51) one finds

$$
\mathrm{d} \mathcal{D}^{\dagger} \wedge \mathrm{d} \mathcal{D}=\left(\begin{array}{ccc}
-q^{-1}\left(\mathrm{~d} \xi_{1} \wedge \mathrm{d} \bar{\xi}_{1}+\mathrm{d} \xi_{2} \wedge \mathrm{d} \bar{\xi}_{2}\right) & -\left(q+q^{-1}\right) \mathrm{d} \xi_{2} \wedge \mathrm{d} \bar{\xi}_{1} & 0 \\
\left(q+q^{-1}\right) \mathrm{d} \xi_{1} \wedge \mathrm{d} \bar{\xi}_{2} & q^{3}\left(\mathrm{~d} \xi_{1} \wedge \mathrm{d} \bar{\xi}_{1}+\mathrm{d} \xi_{2} \wedge \mathrm{d} \bar{\xi}_{2}\right) & 0 \\
0 & 0 & 0
\end{array}\right)
$$

and comparing with $(2.53)$ we see that each entry of $F_{\nabla}$ belongs to the submodule $\Omega_{\mathbb{R}_{\theta}^{4}}^{2,-}$. The anti-selfduality equation for the curvature $F_{\widetilde{\nabla}}$ follows in a completely analogous way: by using the real $\mathcal{G}$-coaction $(2.23)$ one constructs a morphism $\tilde{*}_{\theta}: \Omega_{\widetilde{\mathbb{R}}_{\theta}^{4}}^{2} \rightarrow \Omega_{\widetilde{\mathbb{R}}_{\theta}^{4}}^{2}$ in the category ${ }^{{ }^{\theta}} \mathscr{M}$ with the same formula $(2.52)$ but with affine coordinates $\xi_{i}$ substituted with $\zeta_{i}$, and overall changes of sign reflecting the change of "orientation". The consistency 
condition for the corresponding morphisms on the adjoinment bimodules defines the $\mathcal{G}_{\theta^{-}}$ coequivariant Hodge operator, and hence the instanton equations, on the noncommutative sphere $S_{\theta}^{4}$.

\section{Noncommutative gauge theory partition functions}

In this section we construct smooth moduli spaces for the instanton counting problem on the noncommutative projective plane $\mathbb{C P}_{\theta}^{2}$ and natural bundles over them. We then evaluate various equivariant $\mathrm{K}$-theory partition functions for supersymmetric gauge theories on the complex Moyal plane $\mathbb{C}_{\theta}^{2}$, and compare them with the known results in the commutative case $\theta=0$. In particular, we extend the study of pure gauge theories considered in [6] to gauge theories with both adjoint and fundamental matter fields. Although the technical details of the derivations differ, our noncommutative instanton counting functions coincide with those of the classical case, as desired.

3.1. Instanton moduli spaces. To construct our instanton moduli spaces, we look not for a set of objects but rather for a space which parametrizes those objects. For this, we consider functors from the category $\mathrm{Alg}$ of commutative, unital noetherian $\mathbb{C}$-algebras to the category Set of sets. Let $A$ be a commutative, unital noetherian $\mathbb{C}$-algebra. We write $\mathbb{C P}_{\theta, A}^{2}$ for the noncommutative variety dual to the algebra $\mathcal{A}\left(\mathbb{C P}_{\theta, A}^{2}\right):=A \otimes \mathcal{A}\left(\mathbb{C P}_{\theta}^{2}\right)$.

We consider such families of algebras $\mathcal{A}\left(\mathbb{C P}_{\theta, A}^{2}\right)$ in order to study the global structure of our moduli spaces, and endow them with geometric data. A family of framed sheaves parametrized by the algebra $A$ is an $A$-flat torsion free module $\mathcal{E}$ of rank $r$ on $A \otimes \mathcal{A}\left(\mathbb{C P}_{\theta}^{2}\right)$ together with families of isomorphisms $H^{1}\left(\mathbb{C P}_{\theta, A}^{2}, \mathcal{E}(-1)\right) \cong A \otimes V$ and $\varphi_{A}: \mathcal{E}_{\infty}:=$ $A \otimes i^{\bullet}(E) \rightarrow W \otimes A \otimes \mathcal{O}_{\mathbb{C P}_{\theta}^{1}}$. Two families of framed sheaves $\left(\mathcal{E}, \varphi_{A}\right)$ and $\left(\mathcal{E}^{\prime}, \varphi_{A}^{\prime}\right)$ are isomorphic if they are isomorphic as framed modules on $A \otimes \mathcal{A}\left(\mathbb{C P}_{\theta}^{2}\right)$. For $A=\mathbb{C}$, i.e. for a family parametrized by a one-point space, this definition reduces to our earlier notion of framed sheaf. The instanton moduli functor is the covariant functor $\mathscr{M}_{\text {inst }}(r, k):$ Alg $\rightarrow$ Set that associates to every algebra $A$ the set $\mathscr{M}_{\text {inst }}(r, k)(A)$ of isomorphism classes of families $\left(\mathcal{E}, \varphi_{A}\right)$ of framed sheaves parametrized by $A$, and to every algebra morphism $f: A \rightarrow B$ associates the pushforward $\mathscr{M}_{\text {inst }}(r, k)(f)$ sending families parametrized by $A$ to families parametrized by $B$. A fine instanton moduli space is a variety dual to a universal object representing the instanton moduli functor, i.e. a triple $\left(\hat{A}, \hat{\mathcal{E}}, \hat{\varphi}_{\hat{A}}\right)$, where $\hat{A}$ is an object of the category Alg and $\left(\hat{\mathcal{E}}, \hat{\varphi}_{\hat{A}}\right) \in \mathscr{M}_{\text {inst }}(r, k)(\hat{A})$, such that for any triple $\left(A, \mathcal{E}, \varphi_{A}\right)$ with $A$ an object of $\operatorname{Alg}$ and $\left(\mathcal{E}, \varphi_{A}\right) \in \mathscr{M}_{\text {inst }}(r, k)(A)$ there exists a unique morphism $\alpha \in \operatorname{Hom}_{\text {Alg }}(\hat{A}, A)$ with $\mathscr{M}_{\text {inst }}(r, k)(\alpha)\left(\hat{\mathcal{E}}, \hat{\varphi}_{\hat{A}}\right) \cong\left(\mathcal{E}, \varphi_{A}\right)$. By Yoneda's lemma, a fine instanton moduli space given by $\left(\hat{A}, \hat{\mathcal{E}}, \hat{\varphi}_{\hat{A}}\right)$ corresponds bijectively to a representation of the instanton moduli functor via an isomorphism of functors $\mathscr{H}_{\hat{A}}:=\operatorname{Hom}_{\text {Alg }}(\hat{A},-) \rightarrow$ $\mathscr{M}_{\text {inst }}(r, k)$, with universal framed sheaf represented by $\left(\hat{\mathcal{E}}, \hat{\varphi}_{\hat{A}}\right)=\mathscr{H}_{\hat{A}}(\mathrm{id})$.

The constructions of the previous section have the following properties:

- For any $r \geq 1, k \geq 0$, the quotient variety $\widehat{\mathcal{M}}_{\theta}^{\mathrm{ADHM}}(r, k)$ is a fine instanton moduli space for framed sheaves on Open $\left(\mathbb{C P}_{\theta}^{2}\right)$ of rank $r$ and Euler characteristic $\chi=r-k$.

- For $r \geq 1, k \geq 0$ coprime integers, the instanton moduli space $\mathcal{M}_{\theta}(r, k)$, when non-empty, is a smooth quasi-projective variety of dimension

$$
\operatorname{dim}_{\mathbb{C}}\left(\mathcal{M}_{\theta}(r, k)\right)=2 r k .
$$


- The tangent space to $\mathcal{M}_{\theta}(r, k)$ at a point $[(E, \varphi)]$ is canonically the vector space

$$
T_{[(E, \varphi)]} \mathcal{M}_{\theta}(r, k)=\operatorname{Ext}^{1}(E, E(-1)) .
$$

The proof of these statements can be found in $[6, \S 6.1]$ and it makes use of the moduli space constructions of $[17,7]$. In fact, the noncommutative toric variety $\mathbb{C P}_{\theta}^{2}$ occurs in the universal flat family $\mathcal{A}=\mathcal{A}\left(\mathbb{C P}_{\theta}^{2}\right)$ parametrized by the commutative unital algebra $\mathcal{A}\left(\mathbb{C}^{\times}\right)=\mathbb{C}(t)$ over $\mathbb{C}$ dual to the smooth irreducible curve $\bigwedge^{2} T \cong \mathbb{C}^{\times}$. This family includes the commutative polynomial algebra $\mathcal{A}\left(\mathbb{C P}^{2}\right):=\mathbb{C}\left[w_{1}, w_{2}, w_{3}\right]$ (for $\theta=0$ ). The moduli spaces constructed by Nevins and Stafford in [17] all behave well in this family, and are $\mathbb{C}$-schemes in the usual sense: they are constructed as geometric invariant theory quotients of subvarieties of products of grassmannians.

A much more powerful description of the local geometry of the instanton moduli spaces is provided by the instanton deformation complex. By [6, Thm. 6.14], the K-theory class of the tangent space $T_{[(E, \varphi)]} \mathcal{M}_{\theta}(r, k)$ to the instanton moduli space at a closed point $[(E, \varphi)]=\left[\left(B_{1}, B_{2}, I, J\right)\right]$ is isomorphic to the first cohomology group of the complex

$$
\begin{gathered}
\operatorname{End}_{\mathbb{C}}(V)^{\oplus 2} \\
\oplus \longrightarrow \operatorname{End}_{\mathbb{C}}(V) \stackrel{\mathrm{d} \phi}{\longrightarrow} \operatorname{Hom}_{\mathbb{C}}(W, V) \stackrel{\mathrm{d} \mu}{\longrightarrow} \operatorname{End}_{\mathbb{C}}(V) \longrightarrow 0 \\
\operatorname{Hom}_{\mathbb{C}}(V, W)
\end{gathered}
$$

where the differential $\mathrm{d} \mu$ is the linearization of the braided ADHM equation (2.33) while the differential $\mathrm{d} \phi$ is the linearization of the action (2.34) of the gauge group $\mathrm{GL}(k)$ on $\mathcal{X}(W, V)$.

3.2. Deformations of Hilbert schemes. Instantons of rank one will play a prominent role in our ensuing considerations. A torsion free sheaf $E \in \operatorname{coh}\left(\mathbb{C P}_{\theta}^{2}\right)$ has rank one if and only if $M=\Gamma(E) \in \operatorname{gr}(\mathcal{A})$ is isomorphic to a shift $\mathcal{J}(m)$ of a right ideal $\mathcal{J} \subset \mathcal{A}[5, \S 4.3]$. In this case one may identify the instanton moduli space $\mathcal{M}_{\theta}(1, k)$ with an open subset of the commutative deformation $\mathrm{Hilb}_{\theta}^{k}\left(\mathbb{C P}_{\theta}^{2}\right)$ for $\theta \in \mathbb{C}$, constructed in [17, Cor. 6.6 (1)], of the Hilbert scheme of points $\mathrm{Hilb}^{k}\left(\mathbb{C P}^{2}\right)$ parametrizing zero-dimensional subschemes of degree $k$ in $\mathbb{C P}^{2}$. This is a smooth, projective fine moduli space of dimension $2 k$ for torsion free $\mathcal{A}\left(\mathbb{C P}_{\theta}^{2}\right)$-modules in coh $\left(\mathbb{C P}_{\theta}^{2}\right)$ with rank $r=1$ and Euler characteristic $\chi=1-k$ which also behaves well in the family $\mathcal{A}=\mathcal{A}\left(\mathbb{C P}_{\theta}^{2}\right)$, in the sense described above. In particular, it is irreducible, hence connected, and is non-empty for all $k \geq 0$. De Naeghel and Van den Bergh [7] describe the deformation Hilb ${ }_{\theta}^{k}\left(\mathbb{C P}_{\theta}^{2}\right)$ as the scheme parametrizing torsion free graded $\mathcal{A}$-modules $\mathcal{J}=\bigoplus_{n \geq 0} \mathcal{J}_{n}$ of projective dimension one such that

$$
\operatorname{dim}_{\mathbb{C}}\left(\mathcal{A}_{n}\right)-\operatorname{dim}_{\mathbb{C}}\left(\mathcal{J}_{n}\right)=k \quad \text { for } n \gg 0 .
$$

In particular, the module $\mathcal{J}$ corresponds to an ideal sheaf in the sense of [5, §4.3], and hence corresponds to a closed subscheme of $\mathbb{C P}_{\theta}^{2}$ by $[5$, Thm. 4.10].

In this rank one case there is a bijection between the set of ideals of codimension $k$ in the coordinate algebra $\mathcal{A}\left(\mathbb{C}_{\theta}^{2}\right)$ of the complex Moyal plane and the set of triples $\left(B_{1}, B_{2}, I\right) \in \operatorname{End}_{\mathbb{C}}(V)^{\oplus 2} \oplus \operatorname{Hom}_{\mathbb{C}}(\mathbb{C}, V)$ satisfying the braided ADHM equation

$$
B_{1} B_{2}-q^{-2} B_{2} B_{1}=0,
$$


such that no proper $B_{i}$-invariant subspaces of $V$ contain $I(1)$ for $i=1,2$. We may regard the scheme $\operatorname{Hilb}_{\theta}^{k}\left(\mathbb{C}_{\theta}^{2}\right)$ as a commutative deformation of the resolution of the $k$ th symmetric product singularity $\operatorname{Sym}^{k}\left(\mathbb{C}^{2}\right):=\left(\mathbb{C}^{2}\right)^{k} / S_{k}$ provided by the Hilbert-Chow morphism $\operatorname{Hilb}^{k}\left(\mathbb{C}^{2}\right) \rightarrow \operatorname{Sym}^{k}\left(\mathbb{C}^{2}\right)$, which sends an ideal to its support. For this, we note that $\operatorname{Sym}_{\theta}^{k}\left(\mathbb{C}_{\theta}^{2}\right):=\widehat{\mathcal{M}}_{\theta}^{\mathrm{ADHM}}(0, k)$ for $\theta \in \mathbb{C}$ parametrizes isomorphism classes of $k$ dimensional irreducible representations (3.3) of the complex Moyal plane $\mathbb{C}_{\theta}^{2}$. In particular, it represents the moduli functor Alg $\rightarrow$ Set which sends a $\mathbb{C}$-algebra $A$ to the set of simple $A \otimes \mathcal{A}\left(\mathbb{C}_{\theta}^{2}\right)$-module structures on $A^{\oplus k}$; for $A=\mathbb{C}$ this set consists of isomorphism classes of irreducible representations of $\mathcal{A}\left(\mathbb{C}_{\theta}^{2}\right)$ on the vector space $V=\mathbb{C}^{k}$. Note that since the plane is rigid against commutative deformations, there are no commutative deformations of the Hilbert scheme $\operatorname{Hilb}^{1}\left(\mathbb{C}^{2}\right)=\mathbb{C}^{2}$, and so in the charge one case $k=1$ one has

$$
\operatorname{Hilb}_{\theta}^{1}\left(\mathbb{C}_{\theta}^{2}\right) \cong \mathbb{C}^{2}
$$

as a complex variety for all $\theta \in \mathbb{C}$, in agreement with (2.40).

3.3. Equivariant K-theory partition functions. In the classical case, instanton partition functions of topologically twisted supersymmetric gauge theories are given by integrating suitable equivariant characteristic classes over the instanton moduli spaces [16]; in the following we will treat all of our partition functions within the framework of K-theory, as this is more natural from the point of view of the functorial quantization taken in this paper. The equivariant partition function is then the generating function for the K-theory integrals

$$
Z_{\text {inst }}(r ; Q, z)=\sum_{k=0}^{\infty} Q^{k} \int_{\mathcal{M}_{\theta}(r, k)^{\widetilde{T}}} \omega(z),
$$

where $Q$ is a formal variable and $\omega(z)$ is an equivariant K-theory class depending on the canonical generators $z$ of the coordinate algebra of the torus $\widetilde{T}=T \times\left(\mathbb{C}^{\times}\right)^{r-1}$ for $r \geq 1$, with $T=\left(\mathbb{C}^{\times}\right)^{2}$ the "geometrical" torus used for the deformation of $\mathbb{C P}^{2}$ and of the instanton moduli. The integral is evaluated formally by applying the localization theorem in equivariant K-theory, hence $\int_{\mathcal{M}_{\theta}(r, k)^{\widetilde{T}}} \omega(z)$ is a rational function in the coordinate algebra $\mathcal{A}(\widetilde{T})$, i.e. an element of the quotient field of the representation ring of $\widetilde{T}$. A combinatorial formula for the instanton counting functions can be computed explicitly by classifying the $\widetilde{T}$-fixed points in the instanton moduli space $\mathcal{M}_{\theta}(r, k)$, and computing the Euler characteristic classes of the equivariant normal bundles to the fixed loci of the torus action.

We first describe the natural action of the torus $\widetilde{T}$ and its fixed points on the instanton moduli space. We denote by $z=\left(t_{1}, t_{2}, \rho_{1}, \ldots, \rho_{r}\right)$ the canonical generators of the coordinate algebra of $\widetilde{T}$, where we identify $\left(\mathbb{C}^{\times}\right)^{r-1}$ with the maximal torus of $\operatorname{SL}(r)$ given as the hypersurface $\rho_{1} \cdots \rho_{r}=1$ in $\left(\mathbb{C}^{\times}\right)^{r}$. Using this presentation of $\left(\mathbb{C}^{\times}\right)^{r-1}$, we denote its characters by $m=\left(m_{1}, \ldots, m_{r}\right) \in \mathbb{Z}^{r}$. For any $[(E, \varphi)] \in \mathcal{M}_{\theta}(r, k)$, a natural coaction of the Hopf algebra $\mathcal{H}^{(r)}:=\mathbb{C}\left(\rho_{1}, \ldots, \rho_{r}\right)$ on the framing module $E_{\infty} \cong\left(\mathcal{O}_{\mathbb{C P}_{\theta}^{1}}\right)^{\oplus r}$ is defined by

$$
\Delta_{E_{\infty}}^{(r)}: E_{\infty} \longrightarrow \mathcal{H}^{(r)} \otimes E_{\infty}, \quad \Delta_{E_{\infty}}^{(r)}(f)=\sum_{l=1}^{r} \rho_{l} \otimes f_{l}
$$

for $f=\left(f_{1}, \ldots, f_{r}\right) \in\left(\mathcal{O}_{\mathbb{C P}_{\theta}^{1}}\right)^{\oplus r}$. The coaction of the Hopf algebra $\mathcal{H}_{\theta}$ on the moduli space $\widehat{\mathcal{M}}_{\theta}^{\mathrm{ADHM}}(r, k)$ is given by (2.32). To describe the coaction of $\mathcal{H}^{(r)}$, we represent the linear maps $I$ and $J$ by complex matrices $I=\left(I_{a, l}\right)$ and $J=\left(J_{l, a}\right)$ of sizes $k \times r$ 
and $r \times k$, respectively. Then the left $\mathcal{H}^{(r)}$-coaction on the noncommutative ADHM data $\left(B_{1}, B_{2}, I, J\right)$ is given by

$$
\Delta_{X(W, V)}^{(r)}\left(B_{1}, B_{2}, I, J\right)=\left(1 \otimes B_{1}, 1 \otimes B_{2},\left(\rho_{l}^{-1} \otimes I_{a, l}\right),\left(\rho_{l} \otimes J_{l, a}\right)\right) .
$$

This makes the vector spaces $V, W$ objects and $\left(B_{1}, B_{2}, I, J\right)$ morphisms in the category $\mathcal{H}^{(r)} \mathscr{M}$. By construction, the isomorphism $\mathcal{M}_{\theta}(r, k) \rightarrow \widehat{\mathcal{M}}_{\theta}^{\mathrm{ADHM}}(r, k)$ is $\widetilde{T}$-coequivariant.

A fixed point $[(E, \varphi)] \in \mathcal{M}_{\theta}(r, k)^{\widetilde{T}}$ is an isomorphism class of a framed coequivariant sheaf $(E, \varphi)$ (see $[6, \S 2.5]$ ) which is equiped with a coaction of the Hopf algebra $\widetilde{\mathcal{H}}_{\theta}:=$ $\mathcal{H}_{\theta} \otimes \mathcal{H}^{(r)}$; hence $E$ decomposes into a finite direct sum of torsion free $\mathcal{A}\left(\mathbb{C P}_{\theta}^{2}\right)$-modules graded by the character lattice of the torus $\widetilde{T}$ as

$$
E=\bigoplus_{p \in \mathbb{Z}^{2}} \bigoplus_{m \in \mathbb{Z}^{r}} E(p, m)
$$

The left $\widetilde{\mathcal{H}}_{\theta}$-coactions $\widetilde{\Delta}_{E(p, m)}: E(p, m) \rightarrow \widetilde{\mathcal{H}}_{\theta} \otimes E(p, m)$ are given for $f \in E(p, m)$ by $\widetilde{\Delta}_{E(p, m)}(f)=t^{p} \otimes \rho^{m} \otimes f$. The set of $\widetilde{T}$-fixed points in $\mathcal{M}_{\theta}(r, k)$, i.e. the $\widetilde{\mathcal{H}}_{\theta}$-coinvariants, admits a characterization in terms of Young diagrams. This follows from writing analogous character decompositions for the $\widetilde{\mathcal{H}}_{\theta}$-comodules $V$ and $W$, and using the braided ADHM equation (2.33) together with the stability condition to find a combinatorial characterization of the finite lattice of characters $p=\left(p_{1}, p_{2}\right) \in \mathbb{N}^{2}$ defined by the nontrivial isotopical components $V(p, m) \subset V$ and the non-trivial components of the linear maps $B_{i}(p, m)$ for $i=1,2$. From the discussion in $\S 3.2$, it follows that fixed points $\left[\left(B_{1}, B_{2}, I, J\right)\right] \in \widehat{\mathcal{M}}_{\theta}^{\mathrm{ADHM}}(r, k)^{\widetilde{T}}$ correspond bijectively to ideals of codimension $k$ in the affine coordinate algebra $\mathcal{A}\left(\mathbb{C}_{\theta}^{2}\right)$. In particular, the character decomposition (3.5) truncates to

$$
E=\bigoplus_{l=1}^{r} \mathcal{J}_{l}
$$

where $\mathcal{J}_{l}$ for each $l=1, \ldots, r$ is an $\mathcal{H}_{\theta}$-coequivariant torsion free sheaf of rank one on Open $\left(\mathbb{C P}_{\theta}^{2}\right)$. In this way it is proven in $[6$, Prop. 7.8$]$ that $\mathcal{M}_{\theta}(r, k)^{\widetilde{T}}$ is a finite set in bijective correspondence with length $r$ sequences $\vec{\lambda}=\left(\lambda^{1}, \ldots, \lambda^{r}\right)$ of Young diagrams $\lambda^{l}$ of size $|\vec{\lambda}|:=\sum_{l}\left|\lambda^{l}\right|=k$, where $\left|\lambda^{l}\right|$ is the total number of points in $\lambda^{l}$. This fixed point classification coincides with that of the classical case $\theta=0$ [15, Prop. 2.9]. It can be understood in terms of the noncommutative toric geometry as follows. By [5, Prop. 4.15], $\mathcal{H}_{\theta}$-coequivariant ideal sheaves on Open $\left(\mathbb{C P}_{\theta}^{2}\right)$ are in bijective correspondence with $\mathbb{Z}^{2}$ graded subschemes of $\mathbb{C P}_{\theta}^{2}$, with $\mathbb{Z}^{2}$ the character lattice of $T=\left(\mathbb{C}^{\times}\right)^{2}$; in particular, irreducible subschemes correspond to prime ideals in the spectrum of the homogeneous coordinate algebra $\mathcal{A}\left(\mathbb{C P}_{\theta}^{2}\right)$. Moreover, the $\mathcal{H}_{\theta}$-coinvariant ideals $\mathcal{J} \subset \mathcal{A}\left(\mathbb{C P}_{\theta}^{2}\right)$ are monomial ideals. If $\mathcal{J}$ obeys the condition (3.2), then it determines a finite partition $\lambda_{\mathcal{J}}(k)$ of $k$ by considering lattice points which correspond to monomials not contained in $\mathcal{J}$.

The equivariant characters of the $\widetilde{\mathcal{H}}_{\theta}$-comodules $V=V_{\vec{\lambda}}$ and $W=W_{\vec{\lambda}}$ corresponding to a fixed point parametrized by $\vec{\lambda}$ are given by the elements

$$
\operatorname{ch}_{\widetilde{T}}\left(V_{\vec{\lambda}}\right)=\sum_{l=1}^{r} \sum_{p \in \lambda^{l}} \rho_{l} t_{1}^{1-p_{1}} t_{2}^{1-p_{2}}, \quad \operatorname{ch}_{\widetilde{T}}\left(W_{\vec{\lambda}}\right)=\sum_{l=1}^{r} \rho_{l}
$$

in the representation $\operatorname{ring}$ of $\widetilde{T}$, as in the classical case [15]. The restriction of the instanton deformation complex (3.1) to a $\widetilde{T}$-fixed point $\vec{\lambda}$ is a complex in the category $\widetilde{\mathcal{H}}_{\theta} \mathscr{M}$. The 
computation of the $\widetilde{T}$-equivariant character of the tangent bundle over the instanton moduli space thus proceeds exactly as in the classical case (see [9] and [15, Thm. 2.11]). At a fixed point parametrized by a length $r$ sequence $\vec{\lambda}=\left(\lambda^{1}, \ldots, \lambda^{r}\right)$ of Young diagrams with $|\vec{\lambda}|=k$, one has, in the representation ring of $\widetilde{T}$, the character

$$
\operatorname{ch}_{\widetilde{T}}\left(T_{\vec{\lambda}} \mathcal{M}_{\theta}(r, k)\right)=\sum_{l, l^{\prime}=1}^{r} \rho_{l}^{-1} \rho_{l^{\prime}}\left(\sum_{p \in \lambda^{l}} t_{1}^{p_{1}-\left(\lambda^{l^{\prime}}\right)_{p_{2}}^{t}} t_{2}^{\lambda_{p_{1}}^{l}-p_{2}+1}+\sum_{p^{\prime} \in \lambda^{l^{\prime}}} t_{1}^{\left(\lambda^{l}\right)_{p_{2}^{\prime}}^{t}-p_{1}^{\prime}+1} t_{2}^{p_{2}^{\prime}-\lambda_{p_{1}^{\prime}}^{l^{\prime}}}\right)
$$

where $\lambda_{i}$ and $\lambda_{j}^{t}$ denote respectively the number of points in the $i$-th column and $j$-th row of the Young diagram $\lambda$. In particular, the corresponding top form is the equivariant Euler class of the normal bundle to the fixed point; it is given by

$$
\begin{aligned}
\bigwedge_{-1} T_{\vec{\lambda}}^{*} \mathcal{M}_{\theta}(r, k)=\prod_{l, l^{\prime}=1}^{r} \prod_{p \in \lambda^{l}} & \left(1-\rho_{l} \rho_{l^{\prime}}^{-1} t_{1}^{\left(\lambda^{l^{\prime}}\right)_{p_{2}}^{t}-p_{1}} t_{2}^{p_{2}-\lambda_{p_{1}}^{l}-1}\right) \\
& \times \prod_{p^{\prime} \in \lambda^{l^{\prime}}}\left(1-\rho_{l} \rho_{l^{\prime}}^{-1} t_{1}^{p_{1}^{\prime}-\left(\lambda^{l}\right)_{p_{2}^{\prime}}^{t}-1} t_{2}^{\lambda_{p_{1}^{\prime}}^{l^{\prime}}-p_{2}^{\prime}}\right) .
\end{aligned}
$$

With these ingredients one can now write down equivariant instanton partition functions for noncommutative supersymmetric gauge theories. The equivariant K-theory class $\omega(z)$ is, by the localization theorem, given by the pullback of a class $\tilde{\omega}$ on $\mathcal{M}_{\theta}(r, k)$ which descends from a $\widetilde{T}$-equivariant class, evaluated at the fixed point $\vec{\lambda}$, and divided by the Euler character $\bigwedge_{-1} T_{\vec{\lambda}}^{*} \mathcal{M}_{\theta}(r, k)$. The generic form of the equivariant K-theory partition function is thus

$$
Z_{\text {inst }}(r ; Q, z)=\sum_{k=0}^{\infty} Q^{k} \sum_{\vec{\lambda}:|\vec{\lambda}|=k} \frac{\tilde{\omega}(\vec{\lambda})}{\bigwedge_{-1} T_{\vec{\lambda}}^{*} \mathcal{M}_{\theta}(r, k)} .
$$

In the following we look at some explicit instances and extensions of this K-theory counting function.

3.4. $\mathcal{N}=4$ gauge theory. The simplest example is obtained by taking $\tilde{\omega}$ to be the Euler class of the tangent bundle over $\mathcal{M}_{\theta}(r, k)$, i.e. the class of the virtual bundle

$$
\tilde{\omega}=\sum_{j=0}^{2 r k}(-1)^{j} \bigwedge^{j} T^{*} \mathcal{M}_{\theta}(r, k)
$$

In this case $\omega(z)=1$ (independently of the equivariant parameters $z$ ) and the localization integral simply counts the fixed points of the $\widetilde{T}$-action on the instanton moduli spaces. Then (3.8) results in the Vafa-Witten partition function [19] for $\mathcal{N}=4$ gauge theory given by

$$
Z_{\mathrm{inst}}^{\mathrm{N}=4}(r ; Q)=\sum_{\vec{\lambda}} Q^{|\vec{\lambda}|}=\prod_{n=1}^{\infty} \frac{1}{\left(1-Q^{n}\right)^{r}},
$$

which computes the Euler characteristic of the instanton moduli space. 
3.5. $\mathcal{N}=2$ gauge theory. For $\tilde{\omega}=1$, the expression (3.8) reproduces the K-theory version of Nekrasov's partition function [16] for pure $\mathcal{N}=2$ gauge theory given by

$$
Z_{\mathrm{inst}}^{\mathrm{N}=2}(r ; Q, z)=\sum_{k=0}^{\infty} \sum_{\vec{\lambda}:|\vec{\lambda}|=k} \frac{Q^{|\vec{\lambda}|}}{\bigwedge_{-1} T_{\vec{\lambda}}^{*} \mathcal{M}_{\theta}(r, k)} .
$$

3.6. Adjoint matter fields. By twisting $T \mathcal{M}_{\theta}(r, k)$ with a torus character $m$, the Euler class computes the Chern polynomial of the tangent bundle; the resulting noncommutative gauge theory is called the $\mathcal{N}=2^{*}$ theory and it is a deformation of the $\mathcal{N}=4$ theory obtained by adding a section of the tangent bundle $\operatorname{T\mathcal {M}}_{\theta}(r, k)$, i.e. an adjoint matter field, of mass $m$. We set $\tilde{\omega}=c\left(T \mathcal{M}_{\theta}(r, k), m\right)$, where the Chern polynomial of a vector bundle $\mathcal{V} \rightarrow \mathcal{M}_{\theta}(r, k)$ of rank $n$ is defined by the formula

$$
c(\mathcal{V}, u)=\sum_{i=0}^{n} c_{i}(\mathcal{V}) u^{n-i}
$$

with $c_{i}(\mathcal{V})$ the $i$-th Chern class of $\mathcal{V}$. This class descends from the equivariant class

$$
c_{\widetilde{T}}\left(T \mathcal{M}_{\theta}(r, k), m\right)=\bigwedge_{-1}\left(T \mathcal{M}_{\theta}(r, k) \otimes M\right)_{\widetilde{T} \times T_{m}},
$$

where $M=\mathbb{C}$ is the natural irreducible module over the complex torus $T_{m}=\mathbb{C}^{\times}$ parametrized by $\mu:=\mathrm{e}^{-m}$; the torus $\widetilde{T}\left(\operatorname{resp} . T_{m}\right)$ acts trivially on $M\left(\operatorname{resp} . T \mathcal{M}_{\theta}(r, k)\right)$. The $\widetilde{T} \times T_{m}$-equivariant character at a fixed point parametrized by $\vec{\lambda}$ is a simple modification of (3.7) given by

$$
\operatorname{ch}_{\widetilde{T} \times T_{m}}\left(T_{\vec{\lambda}} \mathcal{M}_{\theta}(r, k) \otimes M\right)=\mu \operatorname{ch}_{\widetilde{T}}\left(T_{\vec{\lambda}} \mathcal{M}_{\theta}(r, k)\right)
$$

and hence the noncommutative $\mathcal{N}=2^{*}$ gauge theory partition function obtained from $(3.8)$ is

$$
Z_{\text {inst }}^{\mathcal{N}=2^{*}}(r ; Q, z ; \mu)=\sum_{k=0}^{\infty} Q^{k} \sum_{\vec{\lambda}:|\vec{\lambda}|=k} \frac{\bigwedge_{-1}\left(T_{\vec{\lambda}}^{*} \mathcal{M}_{\theta}(r, k) \otimes M\right)}{\bigwedge_{-1} T_{\vec{\lambda}}^{*} \mathcal{M}_{\theta}(r, k)}
$$

where

$$
\begin{aligned}
\bigwedge_{-1}\left(T_{\vec{\lambda}}^{*} \mathcal{M}_{\theta}(r, k) \otimes M\right)= & \sum_{j=0}^{2 r k}(-1)^{j} \mu^{j} \operatorname{ch}_{\widetilde{T}}\left(\bigwedge^{j} T^{*} \mathcal{M}_{\theta}(r, k)\right) \\
= & \prod_{l, l^{\prime}=1}^{r} \prod_{p \in \lambda^{l}}\left(1-\mu \rho_{l} \rho_{l^{\prime}}^{-1} t_{1}^{\left(\lambda^{l^{\prime}}\right)_{p_{2}}^{t}-p_{1}} t_{2}^{p_{2}-\lambda_{p_{1}}^{l}-1}\right) \\
& \times \prod_{p^{\prime} \in \lambda^{l^{\prime}}}\left(1-\mu \rho_{l} \rho_{l^{\prime}}^{-1} t_{1}^{p_{1}^{\prime}-\left(\lambda^{l}\right)_{p_{2}^{\prime}}^{t}-1} t_{2}^{\lambda_{p_{1}^{\prime}}^{l^{\prime}}-p_{2}^{\prime}}\right) .
\end{aligned}
$$

This partition function can be regarded as a "homotopy" between (3.9) and (3.10) in the sense that it has the limits

$$
Z_{\mathrm{inst}}^{\mathrm{N}=2^{*}}(r ; Q, z ; \mu=0)=Z_{\mathrm{inst}}^{\mathrm{N}=2}(r ; Q, z), \quad Z_{\mathrm{inst}}^{\mathrm{N}=2^{*}}(r ; Q, z ; \mu=1)=Z_{\mathrm{inst}}^{\mathrm{N}=4}(r ; Q) .
$$


3.7. Dirac bundles and fundamental matter fields. Because of the existence of a universal framed module $\left(\hat{\mathcal{E}}, \hat{\varphi}_{\hat{A}}\right)$ over $\mathcal{A}\left(\mathcal{M}_{\theta}(r, k)\right) \otimes \mathcal{A}\left(\mathbb{C P}_{\theta}^{2}\right)$, we can consider the sheaf $\mathcal{E}$ on $\mathcal{M}_{\theta}(r, k) \times \mathcal{M}_{\theta}\left(r, k^{\prime}\right)$ with fibre over a pair of framed torsion free sheaves $\left((E, \varphi),\left(E^{\prime}, \varphi^{\prime}\right)\right)$ on Open $\left(\mathbb{C P}_{\theta}^{2}\right)$ given by

$$
\left.\mathcal{E}\right|_{\left([(E, \varphi)],\left[\left(E^{\prime}, \varphi^{\prime}\right)\right]\right)}=\operatorname{Ext}^{1}\left(E^{\prime}, E(-1)\right) .
$$

Since the cohomological dimension of the category $\operatorname{coh}\left(\mathbb{C P}_{\theta}^{2}\right)$ is two, by Serre duality it follows that $\operatorname{Ext}^{p}\left(E^{\prime}, E(-1)\right)=0$ for all $p>2[5, \S 6.2]$; this is consistent with the fact that the Koszul algebra $\mathcal{A}\left(\mathbb{C}_{\theta}^{2}\right)$ has projective dimension two, so that its Hochschild cohomology $H H^{p}\left(\mathcal{A}\left(\mathbb{C}_{\theta}^{2}\right), M\right)$ vanishes in degrees $p>2$ for arbitrary $\theta \in \mathbb{C}$ and any $\mathcal{A}\left(\mathbb{C}_{\theta}^{2}\right)$ bimodule $M$. Moreover, it is straightforward to modify the proofs of [17, Lem. 7.14] and [6, Thm. 6.4] (which consider the limiting case $k=k^{\prime}, E=E^{\prime}$ ) to show that $\operatorname{Hom}\left(E^{\prime}, E(-1)\right)=\operatorname{Hom}\left(E^{\prime}, E(-k)\right)=0$ for $k>0$ due to the framing conditions, and that $\operatorname{Ext}^{2}\left(E^{\prime}, E(-1)\right)=0$ for the same reason preceded by (twisted) Serre duality; for $E^{\prime}=\mathcal{O}_{\mathbb{C P}_{\theta}^{2}}$ these vanishing results are the content of [6, Prop. 4.7]. This implies that the vector spaces (3.11) all have the same dimension as $\left([(E, \varphi)],\left[\left(E^{\prime}, \varphi^{\prime}\right)\right]\right)$ varies over $\mathcal{M}_{\theta}(r, k) \times \mathcal{M}_{\theta}\left(r, k^{\prime}\right)$. It follows that $\mathcal{E}$ is a vector bundle over $\mathcal{M}_{\theta}(r, k) \times \mathcal{M}_{\theta}\left(r, k^{\prime}\right)$ whose rank may be computed by using $[17$, Cor. 6.2$]$ to get

$\operatorname{dim}_{\mathbb{C}} \operatorname{Ext}^{1}\left(E^{\prime}, E(-1)\right)=\operatorname{rank}\left(E^{\prime}\right)[\operatorname{rank}(E(-1))-\chi(E(-1))]-\chi\left(E^{\prime}\right) \operatorname{rank}(E(-1))$

with $\chi(E(-1))=-k$ (see [6, Thm. 6.4]); whence the rank of $\mathcal{E}$ is

$$
\operatorname{rank}_{\mathbb{C}}(\mathcal{E})=r\left(k+k^{\prime}\right) .
$$

These are precisely the same arguments which show that the instanton moduli space $\mathcal{M}_{\theta}(r, k)$ is smooth of dimension $2 r k$.

By suitably twisting $\mathcal{E}$ with torus characters, we can compute characteristic classes associated with bifundamental matter fields and use them to build noncommutative linear $\mathcal{N}=2$ quiver gauge theory partition functions. In the limiting case $k=k^{\prime}$, there are two particular restrictions of the vector bundle $\mathcal{E}$ that we are interested in. Firstly, the restriction of $\mathcal{E}$ to the diagonal subspace of $\mathcal{M}_{\theta}(r, k) \times \mathcal{M}_{\theta}(r, k)$ is the tangent bundle $\operatorname{T\mathcal {M}}_{\theta}(r, k)$ of rank $2 r k$ whose sections were studied above. Secondly, the push-forward of $\mathcal{E}$ by the projection of $\mathcal{M}_{\theta}(r, k) \times \mathcal{M}_{\theta}(r, k)$ onto the first factor is a vector bundle $\mathcal{V} \rightarrow \mathcal{M}_{\theta}(r, k)$ of rank $k$ called the Dirac bundle; its fibre over a framed torsion free sheaf $(E, \varphi)$ is $H^{1}\left(\mathbb{C P}_{\theta}^{2}, E(-1)\right) \cong V$. Sections of this bundle, suitably twisted with torus characters, are called fundamental matter fields; we shall now evaluate the corresponding noncommutative gauge theory partition functions (3.8).

For this, we fix a positive integer $n_{f}$ and let $T_{M} \cong\left(\mathbb{C}^{\times}\right)^{n_{f}}$ be the maximal torus of the flavour group $\mathrm{GL}(M) \cong \mathrm{GL}\left(n_{f}\right)$ acting on its fundamental representation $M=\mathbb{C}^{n_{f}}$, with parametrization $\mu_{a}:=\mathrm{e}^{-m_{a}}$ for $a=1, \ldots, n_{f}$. The Euler class $\bigwedge_{-1}(\mathcal{V} \otimes M)$ integrates over $\mathcal{M}_{\theta}(r, k)$ to a (virtual) class of degree $\left(2 r-n_{f}\right) k$. The $\mathcal{N}=2$ gauge theory with fundamental matter fields is said to be "conformal" when $n_{f}=2 r$ and "asymptotically free" when $n_{f}<2 r$; henceforth we assume that $n_{f} \leq 2 r$ in order to avoid the use of virtual classes and virtual localization techniques. We take $\tilde{\omega}$ to descend from the equivariant class

$$
\bigwedge_{-1}(\mathcal{V} \otimes M)_{\widetilde{T} \times T_{M}}=\prod_{a=1}^{n_{f}} c\left(\mathcal{V}, m_{a}\right)
$$


where the torus $\widetilde{T}$ (resp. $T_{M}$ ) acts trivially on $M$ (resp. $\mathcal{V}$ ). The $\widetilde{T} \times T_{M}$-equivariant character at a fixed point parametrized by $\vec{\lambda}$ is a simple modification of the $\widetilde{T}$-equivariant character $\operatorname{ch}_{\widetilde{T}}\left(V_{\vec{\lambda}}\right)$ from (3.6) given by

$$
\operatorname{ch}_{\widetilde{T} \times T_{M}}\left(\mathcal{V}_{\vec{\lambda}} \otimes M\right)=\sum_{a=1}^{n_{f}} \mu_{a} \operatorname{ch}_{\widetilde{T}}\left(V_{\vec{\lambda}}\right)
$$

and whence the noncommutative $\mathcal{N}=2$ gauge theory partition function (3.8) with $n_{f} \leq 2 r$ fundamental matter hypermultiplets is given by

$$
Z_{\mathrm{inst}}^{\mathcal{N}=2, n_{f}}(r ; Q, z ; \mu)=\sum_{k=0}^{\infty} Q^{k} \sum_{\vec{\lambda}:|\vec{\lambda}|=k} \frac{\bigwedge_{-1}\left(\mathcal{V}_{\vec{\lambda}}^{*} \otimes M\right)}{\bigwedge_{-1} T_{\vec{\lambda}}^{*} \mathcal{M}_{\theta}(r, k)}
$$

where

$$
\bigwedge_{-1}\left(\mathcal{V}_{\vec{\lambda}}^{*} \otimes M\right)=\prod_{a=1}^{n_{f}} \prod_{l=1}^{r} \prod_{p \in \lambda^{l}}\left(1-\mu_{a} \rho_{l}^{-1} t_{1}^{p_{1}-1} t_{2}^{p_{2}-1}\right) .
$$

For $\mu_{a}=0$ this reduces to the partition function for the pure $\mathcal{N}=2$ gauge theory (3.10).

\section{Noncommutative Vortices}

One of the most tantalizing extensions of our constructions would be to formulate the counting problem for generalized instantons on noncommutative toric threefolds, and to investigate its connection with a noncommutative version of Donaldson-Thomas theory. However, at present such a higher-dimensional extension is not understood, and we anticipate that much more sophisticated moduli space techniques, such as perfect obstruction theory, will be required; see [4] for a detailed comparison in this context between the instanton counting problems in four and six dimensional topologically twisted supersymmetric gauge theories. On the other hand, non-abelian vortices play the roles of instantons in two dimensions. We will construct noncommutative vortices within our framework via certain reductions of our instanton moduli constructions, inspired by the works [10, 2] which show via D-brane constructions that the moduli spaces of non-abelian vortices in two-dimensional $\mathcal{N}=(2,2)$ supersymmetric gauge theories, with adjoint and fundamental matter fields, can be described as holomorphic submanifolds of the moduli spaces of instantons in four dimensions. We shall also compute the corresponding noncommutative vortex partition functions via localization in equivariant K-theory.

4.1. Vortices on the projective line. Let $\mathcal{H}_{\zeta}=\mathbb{C}(\zeta)$ be the Hopf algebra associated to a complex torus $T_{\zeta}=\mathbb{C}^{\times}$which we make to coact on the homogeneous coordinate algebra of the noncommutative projective plane $\mathcal{A}\left(\mathbb{C P}_{\theta}^{2}\right)$ through

$$
\Delta_{\zeta}\left(w_{1}\right)=1 \otimes w_{1}, \quad \Delta_{\zeta}\left(w_{2}\right)=\zeta \otimes w_{2}, \quad \Delta_{\zeta}\left(w_{3}\right)=1 \otimes w_{3} .
$$

The subalgebra of coinvariants for this $\mathcal{H}_{\zeta}$-coaction, generated by $w_{1}$ and $w_{3}$, is the algebra $\mathcal{A}\left(\mathbb{C P}^{1}\right)$ which is dual to a commutative projective line $\mathbb{C P}^{1}$. Note that such a reduction to a commutative variety is unavoidable, as generic deformations of holomorphic curves are always commutative (see $[6, \S 4.1]$ ). As a toric variety, in this reduction we restrict to the real subalgebra $\mathcal{G}_{t}$ of the Hopf algebra $\mathcal{H}$ with $t:=t_{1}$ and $t_{2}=t^{*}$ (as described after (2.18)), so that the induced coaction of $\mathcal{G}_{t}$ on $\mathcal{A}\left(\mathbb{C P}^{1}\right)$ is given by

$$
\Delta_{t}\left(w_{1}\right)=t \otimes w_{1}, \quad \Delta_{t}\left(w_{3}\right)=1 \otimes w_{3} .
$$


The need to restrict the toric symmetry to $\mathcal{G}_{t}$ will be made apparent below when we deal with the real noncommutative plane.

We study the moduli of sheaves over $\mathcal{A}\left(\mathbb{C P}^{1}\right)$ which are pullbacks of framed torsion free sheaves on $\mathbb{C P}_{\theta}^{2}$ by the algebra inclusion $\mathcal{A}\left(\mathbb{C P}^{1}\right) \hookrightarrow \mathcal{A}\left(\mathbb{C P}_{\theta}^{2}\right)$, which induces a functor $\operatorname{coh}\left(\mathbb{C P}_{\theta}^{2}\right) \rightarrow \operatorname{coh}\left(\mathbb{C P}^{1}\right)$. The $\mathcal{H}_{\zeta^{-}}$coaction on $\mathcal{A}\left(\mathbb{C P}_{\theta}^{2}\right)$ has a natural lift to the instanton moduli space $\mathcal{M}_{\theta}(r, k)$, defined on the braided ADHM data (2.31) by the coaction

$$
\Delta_{\zeta}\left(B_{1}, B_{2}, I, J\right)=\left(1 \otimes B_{1}, \zeta \otimes B_{2}, 1 \otimes I, \zeta \otimes J\right)
$$

The subvariety of coinvariants consists of quadruples $\left(B_{1}=B, 0, I, 0\right)$ and is identified as

$$
X_{\text {vort }}(W, V)=\operatorname{End}_{\mathbb{C}}(V) \oplus \operatorname{Hom}_{\mathbb{C}}(W, V) .
$$

The coaction (4.1) preserves the noncommutative complex ADHM equation (2.33) and the action of the gauge group (2.34). The pullback of the equation (2.33) to the subvariety of coinvariants is a trivial identity: $0=0$. The $\mathrm{GL}(k)$-orbits of the data $(B, I)$ define the vortex moduli space

$$
\mathcal{M}_{\mathrm{vort}}(r, k):=\widehat{\mathcal{M}}_{\theta}^{\mathrm{ADHM}}(r, k)^{T_{\zeta}}=\mathcal{X}_{\mathrm{vort}}(W, V) / \mathrm{GL}(k),
$$

where the quotient is defined by restricting to stable pairs $(B, I)$ such that there are no proper $B$-invariant subspaces of $V$ which contain the image of $I$; this restriction removes the pairs at which the action of the gauge group

$$
g \triangleright(B, I)=\left(g B g^{-1}, g I\right), \quad g \in \mathrm{GL}(k)
$$

is not free. As shown by $[10,8,2]$, this subvariety of the instanton moduli space $\mathcal{M}_{\theta}(r, k)=$ $\widehat{\mathcal{M}}_{\theta}^{\mathrm{ADHM}}(r, k)$ is a complex manifold. The tangent space $T_{[(B, I)]} \mathcal{M}_{\mathrm{vort}}(r, k)$ is described in K-theory by the cohomology of the corresponding pullback of the instanton deformation complex (3.1) which is given by

$$
0 \longrightarrow \operatorname{End}_{\mathbb{C}}(V) \stackrel{\mathrm{d} \phi}{\longrightarrow} \underset{\operatorname{Hom}_{\mathbb{C}}(W, V)}{\oplus} \longrightarrow 0
$$

In particular, the moduli space of vortices has dimension

$$
\operatorname{dim}_{\mathbb{C}}\left(\mathcal{M}_{\mathrm{vort}}(r, k)\right)=r k .
$$

In this way the vortices on $\mathbb{C P}^{1}$ correspond to instantons on $\mathbb{C P}_{\theta}^{2}$ which are invariant under the action of the complex torus $T_{\zeta}$. Moreover, the $\mathcal{G}_{t}$-coaction on $\mathcal{A}\left(\mathbb{C P}^{1}\right)$ also naturally lifts to the vortex moduli space to give the coaction

$$
\Delta_{t}(B, I)=(t \otimes B, 1 \otimes I) .
$$

For example, in the rank one case $r=1$ the vortex moduli space $\mathcal{M}_{\text {vort }}(1, k)$ can be identified with the Hilbert scheme $\mathrm{Hilb}^{k}(\mathbb{C})$ which is the $k$-th symmetric product orbifold $\operatorname{Sym}^{k}(\mathbb{C})=\mathbb{C}^{k} / S_{k}=\mathbb{C}^{k}$. This space is non-singular and one has an isomorphism

$$
\mathcal{M}_{\text {vort }}(1, k) \cong \mathbb{C}^{k}
$$

of smooth complex varieties.

On the other hand, for vorticity $k=1$ we have $B=b \in \mathbb{C}$ and $I=\left(i_{1}, \ldots, i_{r}\right) \in W^{*}$, with the stability condition implying $i_{l} \neq 0$ for at least one index $l=1, \ldots, r$. The action 
of the gauge group $\mathrm{GL}(1)=\mathbb{C}^{\times}$leaves $b$ alone and can be used to rescale $i_{r}=1$, describing a patch $\mathbb{C} \times \mathbb{C}^{r-1}$ of the vortex moduli space, which is therefore given globally by

$$
\mathcal{M}_{\text {vort }}(r, 1) \cong \mathbb{C} \times \mathbb{C P}^{r-1}
$$

as a smooth complex variety. For $r, k>1$, the moduli space $\mathcal{M}_{\text {vort }}(r, k)$ is a resolution of the symmetric product $\operatorname{Sym}^{k}\left(\mathbb{C} \times \mathbb{C P}^{r-1}\right)$.

4.2. Vortices on the noncommutative plane. Despite their origin within the moduli space of noncommutative instantons on $\mathbb{C P}_{\theta}^{2}$, at the level of holomorphic varieties the vortex moduli space essentially coincides with its classical limit; the moduli space $\mathcal{M}_{\text {vort }}(r, k)$ is constructed directly in [8] from a field theory analysis. What differs is its geometry as an embedded submanifold of the instanton moduli space. In the classical case it can be regarded as a symplectic quotient with respect to the natural (hyper-Kähler) symplectic structure on the instanton moduli space [10, 2], while in the noncommutative case the instanton moduli space $\mathcal{M}_{\theta}(r, k)$ is not even a symplectic manifold (at least in the sense of standard hamiltonian reduction); see $[6, \S 6.6]$ for a braided version of symplectic reduction in this context which may illuminate how the noncommutative deformation affects the induced geometry of the smooth variety $\mathcal{M}_{\text {vort }}(r, k)$. Alternatively, we may explore the effects of the quantization by examining the reductions of the instanton moduli on $S_{\theta}^{4}$, which requires introducing suitable real structures. The situation is reminescent of the manner in which dimensional reductions of invariant instantons over the quantum two-sphere yield commutative $q$-deformations of the usual non-abelian vortices [13].

The coaction of the Hopf algebra $\mathcal{H}_{\zeta}$ above has a natural extension to the algebra $\mathcal{A}\left(\mathbb{R}_{\theta}^{4}\right)$ from $\S 2.4$ defined by

$$
\Delta_{\zeta}\left(\xi_{1}\right)=1 \otimes \xi_{1}, \quad \Delta_{\zeta}\left(\xi_{2}\right)=\zeta \otimes \xi_{2}, \quad \Delta_{\zeta}\left(\bar{\xi}_{1}\right)=1 \otimes \bar{\xi}_{1}, \quad \Delta_{\zeta}\left(\bar{\xi}_{2}\right)=\zeta^{*} \otimes \bar{\xi}_{2}
$$

for a suitable $*$-structure on $\mathcal{H}_{\zeta}$ which is compatible with the coaction on $\mathcal{A}\left(\mathbb{R}_{\theta}^{4}\right)$ below defining the desired coinvariants. The subalgebra of coinvariants $\mathcal{A}\left(\mathbb{R}_{\theta}^{2}\right)=\mathcal{A}\left(\mathbb{R}_{\theta}^{4}\right)^{\mathcal{H}_{\zeta}}$ is the algebra dual to a noncommutative real plane $\mathbb{R}_{\theta}^{2}$, generated by elements $\xi, \bar{\xi}$ with commutation relations

and $*$-structure from $(2.22)$,

$$
\xi \bar{\xi}=q^{2} \bar{\xi} \xi
$$

$$
\xi^{\dagger}=q^{-1} \bar{\xi} .
$$

The induced toric coaction $\Delta_{t}: \mathcal{A}\left(\mathbb{R}_{\theta}^{2}\right) \rightarrow \mathcal{G}_{t} \otimes \mathcal{A}\left(\mathbb{R}_{\theta}^{2}\right)$ is given by

$$
\Delta_{t}(\xi)=t \otimes \xi, \quad \Delta_{t}\left(\xi^{\dagger}\right)=t^{*} \otimes \xi^{\dagger},
$$

for the real limit $t=t_{1}$ and $t_{2}=t^{*}$. The algebra inclusion $\mathcal{A}\left(\mathbb{R}_{\theta}^{2}\right) \hookrightarrow \mathcal{A}\left(\mathbb{R}_{\theta}^{4}\right)$ induces a functor $\bmod \left(\mathcal{A}\left(\mathbb{R}_{\theta}^{4}\right)\right) \rightarrow \bmod \left(\mathcal{A}\left(\mathbb{R}_{\theta}^{2}\right)\right)$, giving a map from bundles on $\mathbb{R}_{\theta}^{4}$ to bundles on $\mathbb{R}_{\theta}^{2}$.

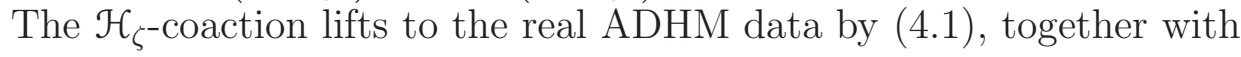

$$
\Delta_{\zeta}\left(B_{1}^{\dagger}, B_{2}^{\dagger}, I^{\dagger}, J^{\dagger}\right)=\left(1 \otimes B_{1}^{\dagger}, \zeta^{*} \otimes B_{2}^{\dagger}, 1 \otimes I^{\dagger}, \zeta^{*} \otimes J^{\dagger}\right)
$$

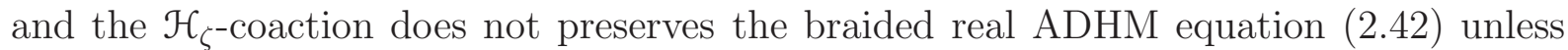
we restrict to the coinvariants for this coaction. The subvariety $\mathcal{M}_{\text {vort }}^{\mathbb{R}}(r, k)_{\theta} \subset \mathcal{M}_{\text {vort }}(r, k)$ consists of pairs $(B, I)$ which additionally satisfy the corresponding pullback of $(2.42)$ given by

$$
\left[B, B^{\dagger}\right]_{-\theta}+I I^{\dagger}=0
$$

in $\operatorname{End}_{\mathbb{C}}(V)$, with $q \in \mathbb{R}$ and together with the pullback of the natural GL( $\left.k\right)$-action on $\mathcal{M}_{\text {vort }}(r, k)$ to an action of the unitary gauge group $\mathrm{U}(k)$. We identify $\mathcal{M}_{\text {vort }}^{\mathbb{R}}(r, k)_{\theta}$ as a 
moduli space of (local) vortices on the noncommutative plane $\mathbb{R}_{\theta}^{2}$. It can be regarded as the phase space of an abstract quantum integrable system; for $r=1$ this integrable system is a $q$-deformation of the Calogero-Moser model.

As an example, consider the vorticity one case. The real ADHM equation (4.5) in this instance reads

$$
\left(1-q^{-2}\right) b \bar{b}+\sum_{l=1}^{r} i_{l} \bar{i}_{l}=0
$$

which has solutions only when $q \in(0,1)$; in this case the real vortex moduli space $\mathcal{M}_{\mathrm{vort}}^{\mathbb{R}}(r, 1)_{\theta}$ is a (resolved) hyperspherical cone in the space (4.4). The set $\mathcal{M}_{\mathrm{vort}}^{\mathbb{R}}(r, 1)_{\theta}$ is empty in the classical case $\theta=0$, in contrast to the corresponding instanton moduli space $\widehat{\mathcal{M}}_{\theta}^{\mathbb{R}}(r, 1)$ which is non-empty for all $q \in \mathbb{R}$.

4.3. Noncommutative vortex counting functions. The $\mathcal{N}=(2,2)$ gauge theory in two dimensions with $r$ fundamental chiral multiplets can be obtained as a dimensional reduction of four-dimensional $\mathcal{N}=1$ supersymmetric Yang-Mills theory. For completeness, we will now work out the various combinatorial vortex partition functions in parallel to what we did in $\S 3$; similar calculations appear for the classical case in $[20,2]$. Following [2], we can interpret these partition functions in terms of surface operators in the original four-dimensional noncommutative $\mathcal{N}=2$ gauge theory, with the two-dimensional gauge theory living on the defect curve $\mathbb{C P}^{1} \subset \mathbb{C P}_{\theta}^{2}$.

As previously, we consider the left $\mathcal{H}^{(r)}$-coaction on the vortex moduli $(B, I)$ given by

$$
\Delta_{X_{\mathrm{vort}}(W, V)}^{(r)}(B, I)=\left(1 \otimes B,\left(\rho_{l}^{-1} \otimes I_{a, l}\right)\right) .
$$

Note that both torus coactions $(2.32)$ and (3.4) are coequivariant for the $\mathcal{H}_{\zeta \text {-coaction }}$ (4.1), i.e. one has

$$
\left(\mathrm{id} \otimes \Delta_{X(W, V)}\right) \circ \Delta_{\zeta}=\Delta_{\zeta} \circ \Delta_{X(W, V)}, \quad\left(\mathrm{id} \otimes \Delta_{X(W, V)}^{(r)}\right) \circ \Delta_{\zeta}=\Delta_{\zeta} \circ \Delta_{X(W, V)}^{(r)} .
$$

It follows that the set of coinvariants $\mathcal{M}_{\text {vort }}(r, k)^{\widetilde{T}}$ for the coaction of $\widetilde{\mathcal{H}}_{t}:=\mathcal{G}_{t} \otimes \mathcal{H}^{(r)}$ can be obtained from the subset of anti-diagonal $\mathcal{H}_{\zeta}$-coinvariants in $\mathcal{M}_{\theta}(r, k)^{\widetilde{T}}$ for $t=t_{1}=t_{2}^{*}$. In particular, the vector space $V$ has the isotopical decomposition

$$
V=\bigoplus_{p \in \mathbb{Z}} \bigoplus_{m \in \mathbb{Z}^{r}} V(p, m)
$$

where here $\mathbb{Z}$ is the character lattice of the torus $T_{t}=\mathbb{C}^{\times}$with parameter $t$. Now only the components of the linear map $B=B_{1}$ are involved with $B(p, m): V(p, m) \rightarrow V(p-1, m)$ for $p \in \mathbb{Z}$ and $m \in \mathbb{Z}^{r}$. It follows that the parametrization of the fixed points in the vortex moduli space are the same as in the instanton moduli space $[6, \S 7.2]$ with a reduction of one of the directions of the character lattice of $T=\left(\mathbb{C}^{\times}\right)^{2}$; they are thus parametrized by $r$-vectors of column Young diagrams, i.e. $r$-component partitions $\vec{k}=\left(k_{1}, \ldots, k_{r}\right)$ of the vorticity $k=|\vec{k}|:=k_{1}+\cdots+k_{r}$, where $k_{l}=\operatorname{dim}_{\mathbb{C}}\left(\bigoplus_{p \in \mathbb{Z}} V(p, l)\right)$ for an $\tilde{\mathcal{H}}_{t}$-comodule $V=V_{\vec{k}}$ corresponding to a fixed point parametrized by $\vec{k}$ with $\operatorname{dim}_{\mathbb{C}}(V(p, l))=0,1$.

The equivariant characters of the $\widetilde{\mathcal{H}}_{t}$-comodules $V=V_{\vec{k}}$ and $W=W_{\vec{k}}$ corresponding to a fixed point parametrized by $\vec{k}$ are given from (3.6) by the elements

$$
\operatorname{ch}_{\widetilde{T}_{t}}\left(V_{\vec{k}}\right)=\sum_{l=1}^{r} \sum_{i=1}^{k_{l}} \rho_{l} t^{1-i}=\sum_{l=1}^{r} \rho_{l} \frac{1-t^{-k_{l}}}{1-t^{-1}}, \quad \operatorname{ch}_{\widetilde{T}_{t}}\left(W_{\vec{k}}\right)=\sum_{l=1}^{r} \rho_{l}
$$


in the representation ring of $\widetilde{T}_{t}=T_{t} \times\left(\mathbb{C}^{\times}\right)^{r-1}$; the dual modules $V_{\vec{k}}^{*}$ and $W_{\vec{k}}^{*}$ transform with inverse weights $\rho_{l}^{-1}$ and $t^{-1}$. From the cohomology of the deformation complex (4.2) one easily computes the $\widetilde{T}_{t}$-equivariant character of the tangent space at a fixed point parametrized by $\vec{k}$ as

$$
\begin{aligned}
\operatorname{ch}_{\widetilde{T_{t}}}\left(T_{\vec{k}} \mathcal{M}_{\text {vort }}(r, k)\right) & =\operatorname{ch}_{\widetilde{T}_{t}}\left(V_{\vec{k}}\right)\left(\operatorname{ch}_{\widetilde{T}_{t}}\left(W_{\vec{k}}^{*}\right)+(t-1) \operatorname{ch}_{\widetilde{T}_{t}}\left(V_{\vec{k}}^{*}\right)\right) \\
& =\sum_{l, l^{\prime}=1}^{r} \rho_{l} \rho_{l^{\prime}}^{-1} \frac{1-t^{-k_{l}}}{1-t^{-1}}\left(1+(t-1) \frac{1-t^{k_{l^{\prime}}}}{1-t}\right) \\
& =\sum_{l, l^{\prime}=1}^{r} \rho_{l}^{-1} \rho_{l^{\prime}} \frac{t^{k_{l}}-t^{k_{l}-k_{l^{\prime}}}}{1-t^{-1}},
\end{aligned}
$$

and hence the corresponding equivariant Euler class is given by

$$
\bigwedge_{-1} T_{\vec{k}}^{*} \mathcal{M}_{\mathrm{vort}}(r, k)=\prod_{l, l^{\prime}=1}^{r}\left(1-\rho_{l} \rho_{l^{\prime}}^{-1} \frac{t^{-k_{l}}-t^{k_{l^{\prime}}-k_{l}}}{1-t}\right) .
$$

Proceeding as in $\S 3$ we can now compute various equivariant K-theory partition functions for vortex counting in the noncommutative gauge theory which by the localization theorem are of the generic form

$$
Z_{\text {vort }}(r ; Q, z)=\sum_{k=0}^{\infty} Q^{k} \int_{\mathcal{M}_{\text {vort }}(r, k)^{\widetilde{T}}} \omega(z)=\sum_{k=0}^{\infty} Q^{k} \sum_{\vec{k}:|\vec{k}|=k} \frac{\tilde{\omega}(\vec{k})}{\bigwedge_{-1} T_{\vec{k}}^{*} \mathcal{M}_{\text {vort }}(r, k)},
$$

where $\omega(z)$ is an equivariant K-theory class depending on $z=\left(t, \rho_{1}, \ldots, \rho_{r}\right)$ which is given by the pullback of a class $\tilde{\omega}$ on $\mathcal{M}_{\text {vort }}(r, k)$; in our examples $\tilde{\omega}$ will be regarded as the pullback to the smooth equivariant embedding $\mathcal{M}_{\text {vort }}(r, k) \subset \mathcal{M}_{\theta}(r, k)$ of the corresponding instanton counting class. Let us now look at some explicit examples of this partition function.

$\mathcal{N}=(4,4)$ gauge theory. By taking $\tilde{\omega}$ to be the Euler class of the tangent bundle $T \mathcal{M}_{\text {vort }}(r, k)$, we recover the enhanced noncommutative $\mathcal{N}=(4,4)$ supersymmetric gauge theory with $r$ fundamental hypermultiplets obtained by dimensional reduction of $\mathcal{N}=4$ gauge theory in four dimensions [2]. In this case the partition function (4.8) computes the Euler characteristic of the vortex moduli space which is given by

$$
Z_{\mathrm{vort}}^{\mathcal{N}=(4,4)}(r ; Q)=\sum_{\vec{k}} Q^{|\vec{k}|}=\frac{1}{(1-Q)^{r}}
$$

$\mathcal{N}=(2,2)$ gauge theory. Setting $\tilde{\omega}=1$ in (4.8) we obtain the K-theory version of the partition function for the $\mathcal{N}=(2,2)$ gauge theory with $r$ fundamental hypermultiplets $[20$, 2] given by

$$
Z_{\mathrm{vort}}^{\mathcal{N}=(2,2)}(r ; Q, z)=\sum_{k=0}^{\infty} Q^{k} \sum_{\vec{k}:|\vec{k}|=k} \prod_{l, l^{\prime}=1}^{r} \frac{1}{1-\rho_{l} \rho_{l^{\prime}}^{-1} \frac{t^{-k_{l}-t^{k} l^{\prime}-k_{l}}}{1-t}} .
$$


Adjoint matter fields. The dimensional reduction of $\mathcal{N}=2^{*}$ gauge theory in four dimensions likewise leads to an analogous field theory in two dimensions [2]. By adding an adjoint matter field of twisted mass $m$ to the $\mathcal{N}=(4,4)$ vector multiplet analogously to $\S 3.6$, i.e. taking $\tilde{\omega}$ to descend from the equivariant class $\bigwedge_{-1}\left(T \mathcal{M}_{\text {vort }}(r, k) \otimes M\right)_{\widetilde{T}_{t} \times T_{m}}$ with $M=\mathbb{C}$, we obtain from (4.8) the partition function for the noncommutative $\mathcal{N}=(2,2)^{*}$ gauge theory given by

$$
Z_{\mathrm{vort}}^{\mathrm{N}=(2,2)^{*}}(r ; Q, z ; \mu)=\sum_{k=0}^{\infty} Q^{k} \sum_{\vec{k}:|\vec{k}|=k} \prod_{l, l^{\prime}=1}^{r} \frac{1-\mu \rho_{l} \rho_{l^{\prime}}^{-1} \frac{t^{-k_{l}}-t^{k_{l^{\prime}}-k_{l}}}{1-t}}{1-\rho_{l} \rho_{l^{\prime}}^{-1} \frac{t^{-k_{l}-t^{k^{\prime}}-k_{l}}}{1-t}} .
$$

As for instanton counting, this partition function continuously interpolates between the partition functions (4.10) at $\mu=0$ and (4.9) at $\mu=1$.

Anti-fundamental matter fields. The $\mathcal{N}=(2,2)$ gauge theory in two dimensions is already endowed with $r$ fundamental matter fields; the coordinates $\left(\rho_{1}, \ldots, \rho_{r}\right)$ of $\left(\mathbb{C}^{\times}\right)^{r-1}$ give twisted mass parameters $a_{l}=-\log \rho_{l}$ to the fundamental multiplets, with $a_{1}+\cdots+a_{r}=$ 0 . We can consider an additional $n_{f}$ anti-fundamental matter fields by pulling back the rank $k$ Dirac bundle $\mathcal{V} \rightarrow \mathcal{M}_{\theta}(r, k)$ from $\S 3.7$ to the smooth equivariant embedding $\iota: \mathcal{M}_{\text {vort }}(r, k) \hookrightarrow \mathcal{M}_{\theta}(r, k)$ of the vortex moduli space; the corresponding Euler class $\bigwedge_{-1}\left(\iota^{*} \mathcal{V} \otimes M\right)$ with $M=\mathbb{C}^{n_{f}}$ now integrates to a stable class provided $n_{f} \leq r$. The fibre of the bundle $\iota^{*} \mathcal{V}$ over a closed point $[(B, I)] \in \mathcal{M}_{\text {vort }}(r, k)$ is the vector space $V$ with

equivariant character at a fixed point parametrized by a partition $\vec{k}$ given in (4.7). With $\tilde{\omega}$ the descendent of the equivariant class $\bigwedge_{-1}\left(\iota^{*} \mathcal{V} \otimes M\right)_{\widetilde{T}_{t} \times T_{M}}$, the $\mathcal{N}=(2,2)$ gauge theory partition function (4.8) with $n_{f} \leq r$ anti-fundamental matter multiplets is given by

$$
Z_{\mathrm{vort}}^{\mathcal{N}=(2,2), n_{f}}(r ; Q, z ; \mu)=\sum_{k=0}^{\infty} Q^{k} \sum_{\vec{k}:|\vec{k}|=k} \frac{\prod_{a=1}^{n_{f}} \prod_{l=1}^{r}\left(1-\mu_{a} \rho_{l}^{-1} \frac{1-t^{k_{l}}}{1-t}\right)}{\prod_{l, l^{\prime}=1}^{r}\left(1-\rho_{l} \rho_{l^{\prime}}^{-1} \frac{t^{-k_{l}}-t^{k_{l^{\prime}}-k_{l}}}{1-t}\right)} .
$$

\section{REFERENCES}

[1] D. Auroux, L. Katzarkov, D. Orlov: Mirror symmetry for weighted projective planes and their noncommutative deformations, Ann. Math. 167 (2008), 867-943.

[2] G. Bonelli, A. Tanzini, J. Zhao: Vertices, vortices and interacting surface operators, J. High Energy Phys. 1206 (2012), 178.

[3] S. J. Brain, G. Landi, W. D. van Suijlekom: Moduli spaces of instantons on toric noncommutative manifolds, preprint arXiv:1204.2148 [math-ph].

[4] M. Cirafici, R. J. Szabo: Curve counting, instantons and McKay correspondences, preprint arXiv:1209.1486 [hep-th].

[5] L. Cirio, G. Landi, R. J. Szabo: Algebraic deformations of toric varieties I. General constructions, preprint arXiv:1001.1242 [math.QA].

[6] L. Cirio, G. Landi, R. J. Szabo: Algebraic deformations of toric varieties II. Noncommutative instantons, Adv. Theor. Math. Phys. 15 (2011), 1817-1907.

[7] K. De Naeghel, M. Van den Bergh: Ideal classes of three-dimensional Artin-Schelter regular algebras, J. Algebra 283 (2005), 399-429.

[8] M. Eto, Y. Isozumi, M. Nitta, K. Ohashi, N. Sakai: Solitons in the Higgs phase: The moduli matrix approach, J. Phys. A 39 (2006), R315-R392. 
[9] R. Flume, R. Poghossian: An algorithm for the microscopic evaluation of the coefficients of the Seiberg-Witten prepotential, Int. J. Mod. Phys. A 18 (2003), 2541-2563.

[10] A. Hanany, D. Tong: Vortices, instantons and branes, J. High Energy Phys. 0307 (2003), 037.

[11] C. Ingalls: Quantum toric varieties, preprint available at http://kappa.math.unb.ca/ ${ }^{\sim}$ colin/research/nctoric.pdf

[12] A. Kapustin, A. Kuznetsov, D. Orlov: Noncommutative instantons and twistor transform, Commun. Math. Phys. 221 (2001), 385-432.

[13] G. Landi, R. J. Szabo: Dimensional reduction over the quantum sphere and non-abelian q-vortices, Commun. Math. Phys. 308 (2011), 365-413.

[14] S. Majid: Foundations of Quantum Group Theory (Cambridge University Press, 1995).

[15] H. Nakajima, K. Yoshioka: Instanton counting on blowup I. 4-dimensional pure gauge theory, Invent. Math. 162 (2005), 313-355.

[16] N. A. Nekrasov: Seiberg-Witten prepotential from instanton counting, Adv. Theor. Math. Phys. 7 (2004), 831-864.

[17] T. A. Nevins, J. T. Stafford: Sklyanin algebras and Hilbert schemes of points, Adv. Math. 210 (2007), 405-478.

[18] R. J. Szabo: Crystals, instantons and quantum toric geometry, Acta Phys. Polon. Supp. 4 (2011), 461-494.

[19] C. Vafa, E. Witten: A strong coupling test of S-duality, Nucl. Phys. B 431 (1994), 3-77.

[20] Y. Yoshida: Localization of vortex partition functions in $\mathcal{N}=(2,2)$ super Yang-Mills theory, preprint arXiv:1101.0872 [hep-th].

University of Luxembourg, Mathematical Research Unit, 6, Rue Richard CoudenhoveKalergi, L-1359 Luxembourg, Grand-Duchy of Luxembourg

Mathematisches Institut, Universität MÜnster, Einsteinstrasse 62, 48149 Münster, GERMAny

E-mail address: lucio.cirio@uni-muenster.de

Dipartimento di Matematica, Università di Trieste, Via A. Valerio 12/1, I-34127 TriESTE, ITALY

infN, Sezione di Trieste, Trieste, Italy

E-mail address: landi@univ.trieste.it

Department of Mathematics, Heriot-Watt University, Colin Maclaurin Building, RicCARTon, Edinburgh EH14 4AS, U.K.

Maxwell Institute for Mathematical Sciences and Tait Institute, Edinburgh, U.K.

E-mail address: R.J.Szabo@hw.ac.uk 